UCB-PTH-03/22

LBNL-53759

hep-th/0309101

\title{
On the Target-Space Geometry of Open-String Orientation-Orbifold Sectors
}

\author{
M.B.Halpern ${ }^{a}$ and C. Helfgott ${ }^{b}$ \\ Department of Physics, University of California and \\ Theoretical Physics Group, Lawrence Berkeley National Laboratory \\ University of California, Berkeley, California 94720, USA
}

\begin{abstract}
Including world-sheet orientation-reversing automorphisms in the orbifold program, we recently reported the twisted operator algebra and twisted KZ equations in each open-string sector of the general WZW orientation orbifold. In this paper we work out the corresponding classical description of these sectors, including the WZW orientation-orbifold actionwhich is naturally defined on the solid half cylinder - and its associated WZW orientationorbifold branes. As a generalization, we also obtain the sigma-model orientation-orbifold action, which describes a much larger class of open-string orientation-orbifold sectors. As special cases, this class includes twisted open-string free boson examples, the open-string WZW sectors above and the open-string sectors of the general coset orientation orbifold. Finally, we derive the orientation-orbifold Einstein equations, in terms of twisted Einstein tensors - which hold when the twisted open-string sigma model sectors are 1-loop conformal.
\end{abstract}

${ }^{a}$ halpern@physics . berkeley . edu

${ }^{b}$ helfgott@socrates. berkeley. edu 


\section{Contents}

1. Introtuention $\quad 3$

2 Gromp Elements and Ging Onbifold Elements 5

2.1 . . . . . . . . . . . . . . . . 5

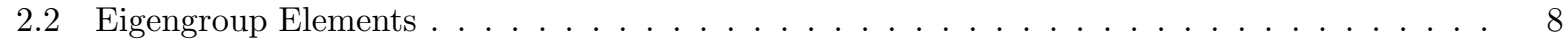

2.8 . . . . . . . . . . . . . 11

2 Aetion and Boundary Condition on the Solid Holf Crlinder 14

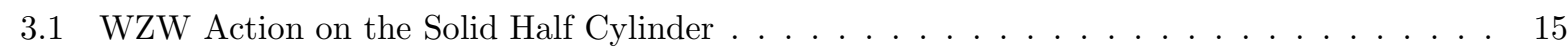

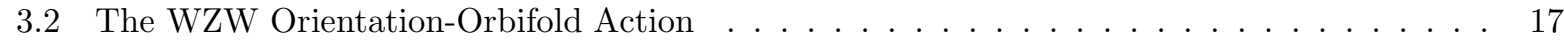

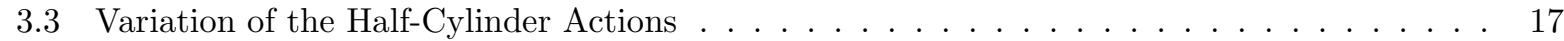

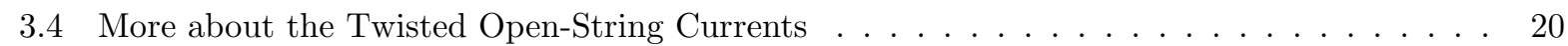

$2.5 h^{2}=1 \ldots \ldots \ldots \ldots \ldots \ldots \ldots$

4 Einstin Geometn the Solid Half Gilinden 25

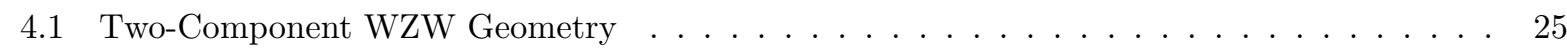

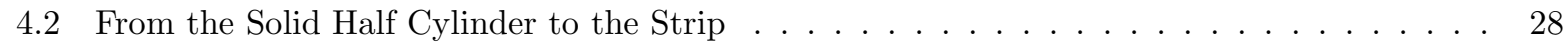

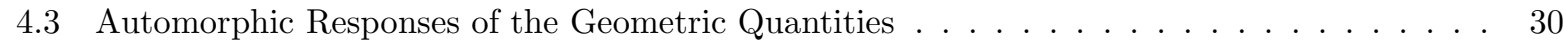

4.4 . . . . . . . . . . . . . . . . . . 31

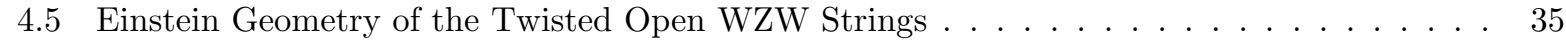

4.6 . $\ldots \ldots \ldots \ldots$

5 Simadeloniention Orbifolds 41

5.1 . . . . . . . . . . . . . . . 41

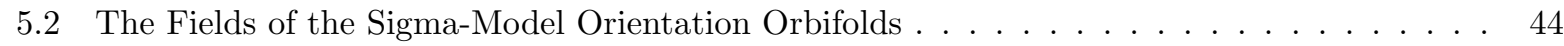

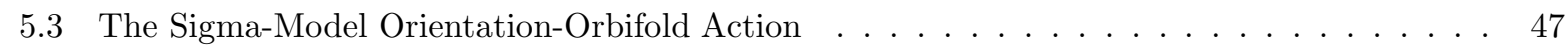

- $\ldots \ldots \ldots \ldots \ldots \ldots$

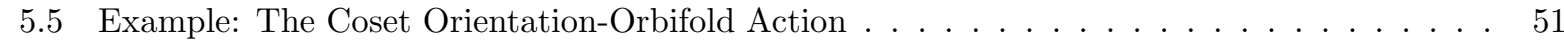

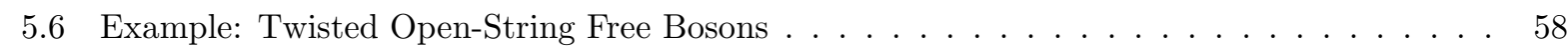

6 The Orientation-Orbifold Einstein Equations $\quad 60$

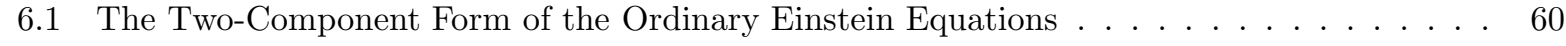

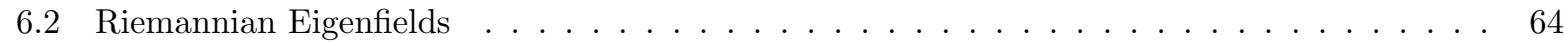

$6.2+\ldots \ldots \ldots \ldots \ldots$

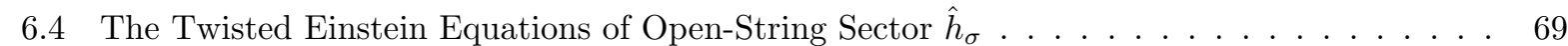

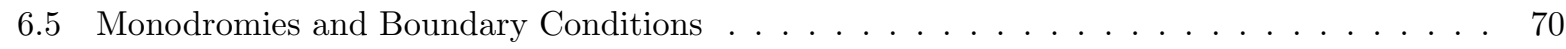

7 Disenssion $\quad 71$

$\begin{array}{ll}\text { A. Useful Identitieg } & 72\end{array}$

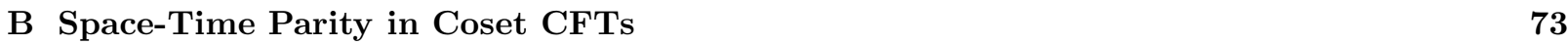

$\begin{array}{ll}\text { Referees } & 74\end{array}$ 


\section{Introduction}

In the last few years there has been a quiet revolution in the local theory of current-algebraic orbifolds. Building on the discovery of orbifold affine algebras [1, 2, in the cyclic permutation orbifolds, Refs. [3-5] gave the twisted currents and stress tensor in each twisted sector of any current-algebraic orbifold $A(H) / H$ - where $A(H)$ is any current-algebraic conformal field theory [6-13] with a discrete symmetry group $H$. The construction treats all currentalgebraic orbifolds at the same time, using the method of eigenfields and the principle of local isomorphisms to map OPEs in the symmetric theory to OPEs in the orbifold. The orbifold results are expressed in terms of a set of twisted tensors or duality transformations, which are discrete Fourier transforms constructed from the eigendata of the $H$-eigenvalue problem.

More recently, the special case of the WZW orbifolds

$$
\frac{A_{g}(H)}{H}, \quad H \subset A u t(g)
$$

was worked out in further detail [14-17], introducing the extended $H$-eigenvalue problem and the linkage relation to include the twisted affine primary fields, the twisted vertex operator equations and the twisted $K Z$ equations of the WZW orbifolds. Ref. [17] includes a review of the general left- and right-mover twisted KZ systems. For detailed information on particular classes of WZW orbifolds, we direct the reader to the following references:

- the WZW permutation orbifolds [14-16]

- the inner-automorphic WZW orbifolds [14, 16]

- the (outer-automorphic) charge conjugation orbifold on $\mathfrak{s u}(n \geq 3)$ [15]

- the outer-automorphic WZW orbifolds on $\mathfrak{s o}(2 n)$, including the triality orbifolds on $\mathfrak{s o}(8)$ [17].

Ref. [15] also solved the twisted vertex operator equations and the twisted $\mathrm{KZ}$ systems in an abelian limit ${ }^{\ddagger 1}$ to obtain the twisted vertex operators for each sector of a large class of orbifolds on abelian $g$. Moreover, Ref. [16] found the general orbifold Virasoro algebra (twisted Virasoro operators [1, 19]) of the WZW permutation orbifolds and used the general twisted KZ system to study reducibility of the general twisted affine primary field. Recent progress at the level of characters has been also reported in Refs. 20, 1, 21, 22,

In addition to the operator formulation, there have been a number of discussions of orbifold geometry at the action level. In particular, Ref. 14 also gave the general WZW orbifold action, special cases of which are further discussed in Refs. [15, 17]. The general

\footnotetext{
${ }^{\ddagger 1} \mathrm{An}$ abelian twisted KZ equation for the inversion orbifold $x \rightarrow-x$ was given earlier in Ref. [18].
} 
WZW orbifold action provides the classical description of each sector of every WZW orbifold in terms of the so-called group orbifold elements with diagonal monodromy, which are the classical (high-level) limit of the twisted affine primary fields. Moreover, Ref. 23] gauged the general WZW orbifold action by general twisted gauge groups to obtain the general coset orbifold action, which describes each sector of the general coset orbifold $A_{g / h}(H) / H$. Finally, the geometric description was extended in Ref. 24] to include a large class of sigma-model orbifolds and their corresponding twisted Einstein equations.

In Ref. 25], the orbifold program was extended beyond the space-time orbifolds above to construct a new class of orbifolds called the WZW orientation orbifolds

$$
\frac{A_{g}\left(H_{-}\right)}{H_{-}}, \quad H_{-} \subset \operatorname{Aut}(g \oplus g)
$$

where $H_{-}$is any automorphism group which contains world-sheet orientation- reversing automorphisms. The orientation-orbifold sectors which arise by twisting the orientationreversing automorphisms are twisted open WZW strings, each of which comes with an extended Virasoro algebra (which is in fact an orbifold Virasoro algebra) at twice the original closed-string central charge. The twisted open-string KZ equations of the WZW orientation orbifolds combine salient features of the untwisted open-string KZ equations [26] and the closed-string twisted KZ equations [14-17] of space-time orbifold theory.

The present paper supplements the operator construction of Ref. 25] by discussing the target-space geometry of open-string orientation orbifold sectors. In particular, we begin by constructing the $W Z W$ orientation orbifold action for each open-string sector of $A_{g}\left(H_{-}\right) / H_{-}$. This action, which is a functional of appropriate group orbifold elements, is naturally defined on the solid half-cylinder. In a generalization, we also obtain the sigma-model orientation orbifold action, which describes a much larger set of open-string orientation orbifold sectors - including the open-string WZW orientation orbifold sectors as well as the open-string sectors of the general coset orientation orbifold. The corresponding orientation-orbifold Einstein equations are also worked out - which hold when the twisted open-string sigma model sectors are one-loop conformal.

The less technically-inclined reader may prefer to look first at Subsec. 5.6, where we discuss the simple case of twisted open-string free boson actions - which result from the twisting of closed strings on abelian $g$. 


\section{Group Elements and Group Orbifold Elements}

\subsection{Orientation-Reversing Automorphisms}

To fix notation, we begin with the affine Lie algebra [27, 28, 6, 12, associated to $\operatorname{Lie}(g \oplus g)$

$$
\begin{gathered}
{\left[J_{a}(m), J_{b}(n)\right]=i f_{a b}^{c} J_{c}(m+n)+m G_{a b} \delta_{m+n, 0}} \\
{\left[\bar{J}_{a}(m), \bar{J}_{b}(n)\right]=i f_{a b}{ }^{c} \bar{J}_{c}(m+n)+m G_{a b} \delta_{m+n, 0}} \\
{\left[J_{a}(m), \bar{J}_{b}(n)\right]=0, \quad m, n \in \mathbb{Z}, \quad a, b, c=1, \ldots, \operatorname{dim} g} \\
g=\oplus_{I} \mathfrak{g}^{I}, \quad f_{a b}{ }^{c}=\oplus_{I} f_{a(I), b(I)}^{I}{ }^{c(I)}, \quad G_{a b}=\oplus_{I} k_{I} \eta_{a(I), b(I)}^{I}
\end{gathered}
$$

where $\mathfrak{g}^{I}$ is any simple compact Lie algebra, $f_{a b}{ }^{c}$ are the structure constants of $g$ and $G_{a b}$ is the generalized metric of affine $g$. The local currents on the cylinder $0 \leq \xi \leq 2 \pi$ corresponding to these modes are:

$$
J_{a}(\xi, t)=\sum_{m} J_{a}(m) e^{-i m(t+\xi)}, \quad \bar{J}_{a}(\xi, t)=\sum_{m} \bar{J}_{a}(m) e^{-i m(t-\xi)} .
$$

For the classical dynamics of this paper, we will need the standard ${ }^{\ddagger 2}$ equal-time bracket form of affine $(g \oplus g)$, including the brackets with the WZW group elements $g(T)$

$$
\begin{gathered}
\left\{J_{a}(\xi, t), J_{b}(\eta, t)\right\}=2 \pi i\left(f_{a b}{ }^{c} J_{c}(\eta, t)+G_{a b} \partial_{\xi}\right) \delta(\xi-\eta) \\
\left\{\bar{J}_{a}(\xi, t), \bar{J}_{b}(\eta, t)\right\}=2 \pi i\left(f_{a b}{ }^{c} \bar{J}_{c}(\eta, t)-G_{a b} \partial_{\xi}\right) \delta(\xi-\eta) \\
\left\{J_{a}(\xi, t), \bar{J}_{b}(\eta, t)\right\}=0 \\
\left\{J_{a}(\xi, t), g(T, \eta, t)\right\}=2 \pi g(T, \eta, t) T_{a} \delta(\xi-\eta) \\
\left\{\bar{J}_{a}(\xi, t), g(T, \eta, t)\right\}=-2 \pi T_{a} g(T, \eta, t) \delta(\xi-\eta) \\
\left\{J_{a}(\xi, t), g^{-1}(T, \eta, t)\right\}=-2 \pi T_{a} g^{-1}(T, \eta, t) \delta(\xi-\eta) \\
\left\{\bar{J}_{a}(\xi, t), g^{-1}(T, \eta, t)\right\}=2 \pi g^{-1}(T, \eta, t) T_{a} \delta(\xi-\eta) \\
\left.T T_{a}, T_{b}\right]=i f_{a b}{ }^{c} T_{c}, \quad T r\left(M T_{a} T_{b}\right)=G_{a b}, \quad\left[M, T_{a}\right]=0, \quad[M, g(T, \xi, t)]=0 \\
g(T)=\left\{g(T)_{\alpha}{ }^{\beta}\right\}, \quad T_{a}=\left\{\left(T_{a}\right)_{\alpha}{ }^{\beta}\right\}, \quad \alpha, \beta=1, \ldots, \operatorname{dim} T
\end{gathered}
$$

where $T$ is any matrix rep of Lie $g$. As shown in Eq. (2.3h), the representation matrices $T$ are normalized with the invertible data matrix $\mathrm{M}[14]$

$$
M \equiv M(k, T)=\oplus_{I} \frac{k_{I}}{y\left(T^{I}\right)}, \quad T=\oplus_{I} T^{I}, \quad g(T, \xi, t)=\oplus_{I} g^{I}\left(T^{I}, \xi, t\right)
$$

\footnotetext{
$\ddagger 2$ Our brackets are rescaled by an $i$ so that $[,] \rightarrow\{$,$\} .$
} 
which records the affine levels $\{k\}$ of each simple component of $g$ and the Dynkin indices $\{y\}$ of rep $T$. The group elements are $2 \pi$-periodic

$$
g(T, \xi+2 \pi, t)=g(T, \xi, t)
$$

and we will also introduce the following tangent-space form of the group elements

$$
g(T, \xi, t)=e^{i \beta^{a}(\xi, t) T_{a}}, \quad \beta^{a}(\xi+2 \pi, t)=\beta^{a}(\xi, t)
$$

where $\beta^{a}, a=1 \ldots \operatorname{dim} g$ are the tangent-space coordinates.

Then the classical (high-level) limit of the affine primary fields $g(T, \bar{z}, z), g(\bar{T}, z, \bar{z})^{T}$ of Ref. [25] on the sphere translate into the following forms of the group elements on the cylinder

$$
\begin{gathered}
g(T, \bar{z}, z) \longrightarrow g(T, \xi, t)=e^{i \beta^{a}(\xi, t) T_{a}} \\
g(\bar{T}, z, \bar{z})^{t} \longrightarrow g(\bar{T},-\xi, t)^{t}=e^{i \beta^{a}(-\xi, t) \bar{T}_{a}^{t}}=e^{-i \beta^{a}(-\xi, t) T_{a}}=g^{-1}(T,-\xi, t) \\
\bar{T} \equiv-T^{t}, \quad\left(T_{a}^{t}\right)_{\alpha}{ }^{\beta} \equiv\left(T_{a}\right)_{\beta}{ }^{\alpha}
\end{gathered}
$$

where superscript $t$ is matrix transpose.

With the correspondence (2.7), we may then read off from Ref. 25] the cylinder form of the general world-sheet orientation-reversing automorphism $\hat{h}_{\sigma}=\tau_{1} \times h_{\sigma} \in H_{-}$of affine $(g \oplus g)$

$$
\begin{gathered}
g(T, \xi, t)^{\prime}=W\left(h_{\sigma} ; T\right) g^{-1}(T,-\xi, t) W^{\dagger}\left(h_{\sigma} ; T\right) \\
g^{-1}(T,-\xi, t)^{\prime}=W\left(h_{\sigma} ; T\right) g(T, \xi, t) W^{\dagger}\left(h_{\sigma} ; T\right) \\
\beta^{a}(\xi, t)^{\prime}=-\beta^{b}(-\xi, t) \omega^{\dagger}\left(h_{\sigma}\right)_{b}{ }^{a}, \quad \beta^{a}(-\xi, t)^{\prime}=-\beta^{b}(\xi, t) \omega^{\dagger}\left(h_{\sigma}\right)_{b}{ }^{a} \\
J_{a}(\xi, t)^{\prime}=\omega\left(h_{\sigma}\right)_{a}{ }^{b} \bar{J}_{b}(-\xi, t), \quad \bar{J}_{a}(\xi, t)^{\prime}=\omega\left(h_{\sigma}\right)_{a}{ }^{b} J_{b}(-\xi, t) \\
\omega\left(h_{\sigma}\right)_{a}{ }^{c} \omega\left(h_{\sigma}\right)_{b}{ }^{d} G_{c d}=G_{a b}, \quad \omega\left(h_{\sigma}\right)_{a}{ }^{d} \omega\left(h_{\sigma}\right)_{b}{ }^{e} f_{d e}{ }^{c}=f_{a b}{ }^{d} \omega\left(h_{\sigma}\right)_{d}{ }^{c} \\
W^{\dagger}\left(h_{\sigma} ; T\right) T_{a} W\left(h_{\sigma} ; T\right)=\omega\left(h_{\sigma}\right)_{a}{ }^{b} T_{b} \\
\omega\left(h_{\sigma}\right) \omega\left(h_{\sigma}\right)^{\dagger}=\mathbb{1}, \quad W\left(h_{\sigma} ; T\right) W^{\dagger}\left(h_{\sigma} ; T\right)=\mathbb{1} \\
{\left[W\left(h_{\sigma} ; T\right), M(k, T)\right]=0}
\end{gathered}
$$

where $\omega\left(h_{\sigma}\right), W\left(h_{\sigma} ; T\right)$ are the actions of $h_{\sigma} \in A u t(g)$ in the adjoint rep and in matrix rep $T$ respectively. The linkage relation [14] in Eq. (2.8f) guarantees the consistency 
of Eqs. (2.8a,b) with (2.8c) and (2.8d). In what follows, we deal exclusively with the orientation-reversing automorphisms (2.8), which lead to open-string sectors. We remind the reader however that $H_{-}$always contains an equal number of orientation-preserving automorphisms, which leads to an equal number of closed-string sectors in each orientation orbifold.

Following the two-component notation of Ref. [25], we can further define a matrix group element and its tangent-space coordinates:

$$
\begin{aligned}
& \tilde{g}(T, \xi, t) \equiv\left(\begin{array}{cc}
g(T, \xi, t) & 0 \\
0 & g^{-1}(T,-\xi, t)
\end{array}\right)=e^{i \beta^{a \dot{I}}(\xi, t) T_{a} \rho_{\dot{I}}} \\
& \rho_{0}=\left(\begin{array}{ll}
1 & 0 \\
0 & 0
\end{array}\right), \quad \rho_{1}=\left(\begin{array}{cc}
0 & 0 \\
0 & 1
\end{array}\right), \quad \operatorname{Tr}\left(\rho_{\dot{I}} \rho_{\dot{J}}\right)=\delta_{\dot{I} \dot{J}}, \quad \rho_{\dot{I}} \rho_{\dot{J}}=\delta_{\dot{I} \dot{J}} \rho_{\dot{I}} \\
& \beta^{a, 0}(\xi, t) \equiv \beta^{a}(\xi, t), \quad \beta^{a, 1}(\xi, t) \equiv-\beta^{a}(-\xi, t), \quad \beta^{a \dot{I}}(\xi+2 \pi, t)=\beta^{a \dot{I}}(\xi, t) \\
& \tilde{g}(T,-\xi, t)=\tau_{1} \tilde{g}^{-1}(T, \xi, t) \tau_{1} \\
& \beta^{a \dot{I}}(-\xi, t)=-\beta^{a \dot{J}}(\xi, t)\left(\tau_{1}\right)_{\dot{J}}^{\dot{I}} \\
& {\left[\tau_{3}, \tilde{g}(T, \xi, t)\right]=\left[M \otimes \mathbb{1}_{2}, \tilde{g}(T, \xi, t)\right]=0} \\
& \tilde{g}(T, \xi, t)^{\prime}=\tau_{1} W\left(h_{\sigma} ; T\right) \tilde{g}(T, \xi, t) W^{\dagger}\left(h_{\sigma} ; T\right) \tau_{1} \\
& \tilde{g}^{-1}(T, \xi, t)^{\prime}=\tau_{1} W\left(h_{\sigma} ; T\right) \tilde{g}^{-1}(T, \xi, t) W^{\dagger}\left(h_{\sigma} ; T\right) \tau_{1} \\
& \beta^{a \dot{I}}(\xi, t)^{\prime}=\beta^{b \dot{J}}(\xi, t) \omega^{\dagger}\left(h_{\sigma}\right)_{b}{ }^{a}\left(\tau_{1}\right)_{\dot{J}}^{\dot{I}} \\
& W^{\dagger}\left(h_{\sigma} ; T\right) T_{a} W\left(h_{\sigma} ; T\right)=\omega\left(h_{\sigma}\right)_{a}{ }^{b} T_{b}, \quad \tau_{1} \rho_{\dot{I}} \tau_{1}=\left(\tau_{1}\right)_{\dot{I} j} \rho_{j}, \quad \dot{I}, \dot{J} \in\{0,1\}
\end{aligned}
$$

Here $\vec{\tau}$ are the Pauli matrices, and the automorphic response (2.9g) of the matrix group element is the same as that of the matrix affine primary field in Ref. 25]. The linkage relations in (2.10) guarantee the consistency of Eqs. $(2.9 \mathrm{~g}, \mathrm{~h})$ with (2.9i). The corresponding two-component form $J_{a \dot{I}}(\xi, t)=\left(J_{a}(\xi, t), \bar{J}_{a}(-\xi, t)\right)$ of the currents is discussed in Ref. [25].

We call attention to the behavior under world-sheet parity $\xi \rightarrow-\xi$ of the matrix group element in (2.9d) and the tangent-space coordinates in (2.9e). These relations tell us that the strip $0 \leq \xi \leq \pi$ is the natural fundamental range for the two-component variables. In what follows we often suppress the time label $t$. 


\subsection{Eigengroup Elements}

The orientation-reversing automorphisms in Eq. (2.9) are now in the proper form required for the orbifold program, which follows the dotted line in the commuting diagram 3, 24, of Fig. 11.

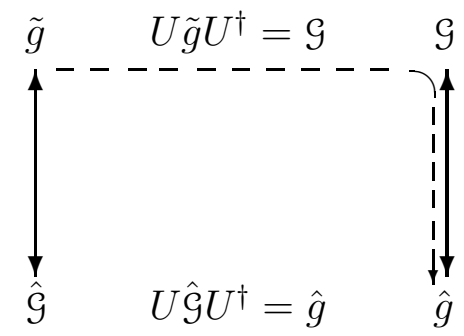

Each vertical double arrow is a local isomorphism $\tilde{g}=$ Lie group elements: mixed under automorphisms

$\mathcal{G}=$ eigengroup elements: diagonal under automorphisms

$\hat{g}=$ group orbifold elements

$\hat{\mathcal{G}}=$ group orbifold elements with twisted boundary conditions

Fig.1] Group and group orbifold elements

In particular, our next task is the construction of the eigenfields $\mathcal{G}$, for which we will need the analogues of the $H$-eigenvalue problem of Ref. [5] and the extended $H$-eigenvalue problem of Ref. [14]:

$$
\begin{gathered}
\omega\left(h_{\sigma}\right) U^{\dagger}(\sigma)=U^{\dagger}(\sigma) E(\sigma), \quad E(\sigma)_{n(r) \mu}^{n(s) \nu}=\delta_{n(r) \mu}{ }^{n(s) \nu} E_{n(r)}(\sigma) \\
W\left(h_{\sigma} ; T\right) U^{\dagger}(T, \sigma)=U^{\dagger}(T, \sigma) E(T, \sigma), \quad E(T, \sigma)_{N(r) \mu}^{N(s) \nu} \equiv \delta_{N(r) \mu}^{N(s) \nu} E_{N(r)}(T, \sigma) \\
\delta_{n(r) \mu}{ }^{n(s) \nu} \equiv \delta_{\mu}{ }^{\nu} \delta_{n(r)-n(s), 0 \bmod \rho(\sigma)}, \quad \delta_{N(r) \mu}^{N(s) \nu} \equiv \delta_{\mu}{ }^{\nu} \delta_{N(r)-N(s), 0 \bmod R(\sigma)} \\
U^{\dagger}(\sigma)=\left\{U^{\dagger}(\sigma)_{a}^{n(r) \mu}\right\}, \quad U^{\dagger}(T, \sigma)=\left\{U^{\dagger}(T, \sigma)_{\alpha}^{N(r) \mu}\right\} \\
E_{n(r)}(\sigma) \equiv e^{-2 \pi i \frac{n(r)}{\rho(\sigma)}}, \quad E_{N(r)}(T, \sigma) \equiv e^{-2 \pi i \frac{N(r)}{R(\sigma)}}
\end{gathered}
$$

Here $\rho(\sigma)$ and $R(\sigma)$ are the orders of $h_{\sigma}$ in the adjoint rep and rep $T$ respectively. The unitary eigenvector matrices $U^{\dagger}(\sigma), U^{\dagger}(T, \sigma)$ are periodic

$$
n(r) \rightarrow n(r) \pm \rho(\sigma), \quad N(r) \rightarrow N(r) \pm R(\sigma)
$$

in their respective spectral indices $n(r)$ and $N(r)$, and the same is true below for any object with these indices. In what follows, the barred quantities $\bar{n}(r)$ and $\bar{N}(r)$ are the pullbacks of the spectral indices to their fundamental ranges

$$
\begin{array}{cl}
\frac{\bar{n}(r)}{\rho(\sigma)}=\frac{n(r)}{\rho(\sigma)}-\left\lfloor\frac{n(r)}{\rho(\sigma)}\right\rfloor, & \frac{\bar{N}(r)}{R(\sigma)}=\frac{N(r)}{R(\sigma)}-\left\lfloor\frac{N(r)}{R(\sigma)}\right\rfloor \\
\bar{n}(r) \in\{0, \ldots, \rho(\sigma)-1\}, & \bar{N}(r) \in\{0, \ldots, R(\sigma)-1\}
\end{array}
$$


where $\lfloor x\rfloor$ is the floor of $x$. Explicit solutions of these eigenvalue problems are given in Refs. [3,5,14-17].

For this discussion, we will also need the standard duality transformations [3, 5, 14, or twisted tensors of sector $\sigma$

$$
\begin{gathered}
\mathcal{G}_{n(r) \mu ; n(s) \nu}(\sigma) \equiv \chi(\sigma)_{n(r) \mu} \chi(\sigma)_{n(s) \nu} U(\sigma)_{n(r) \mu}{ }^{a} U(\sigma)_{n(s) \nu}{ }^{b} G_{a b} \\
=\mathcal{G}_{n(s) \nu ; n(r) \mu}(\sigma)=\delta_{n(r)+n(s), 0 \bmod \rho(\sigma)} \mathcal{G}_{n(r) \mu ;-n(r), \nu}(\sigma) \\
\mathcal{F}_{n(r) \mu ; n(s) \nu}{ }^{n(t) \delta}(\sigma) \equiv \chi(\sigma)_{n(r) \mu} \chi(\sigma)_{n(s) \nu} \chi(\sigma)_{n(t) \delta}^{-1} U(\sigma)_{n(r) \mu}{ }^{a} U(\sigma)_{n(s) \nu}{ }^{b} f_{a b}{ }^{c} U^{\dagger}(\sigma)_{c}{ }^{n(t) \delta} \\
=-\mathcal{F}_{n(s) \nu ; n(r) \mu}{ }^{n(t) \delta}(\sigma)=\delta_{n(r)+n(s)-n(t), 0 \bmod \rho(\sigma)} \mathcal{F}_{n(r) \mu ; n(s) \nu}{ }^{n(r)+n(s), \delta}(\sigma) \\
\mathcal{T}_{n(r) \mu}(T, \sigma) \equiv \chi(\sigma)_{n(r) \mu} U(\sigma)_{n(r) \mu}{ }^{a} U(T, \sigma) T_{a} U^{\dagger}(T, \sigma) \\
E_{n(r)}(\sigma)^{*} \mathcal{T}_{n(r) \mu}(T, \sigma)=E(T, \sigma) \mathcal{T}_{n(r) \mu}(T, \sigma) E^{*}(T, \sigma) \\
{\left[\mathcal{T}_{n(r) \mu}(T, \sigma), \mathcal{T}_{n(s) \nu}(T, \sigma)\right]=i \mathcal{F}_{n(r) \mu ; n(s) \nu}{ }^{n(r)+n(s), \delta}(\sigma) \mathcal{T}_{n(r)+n(s), \delta}(T, \sigma)} \\
\mathcal{M}(\mathcal{T}(T, \sigma), \sigma) \equiv U(T, \sigma) M(k, T) U^{\dagger}(T, \sigma) \\
\left.\left.\widehat{\operatorname{Tr}}(\mathcal{M}(T, \sigma), \sigma), \mathcal{T}_{n(r) \mu}(T, \sigma)\right]=[\mathcal{M}(T, \sigma), \sigma) \mathcal{T}_{n(r) \mu}(T, \sigma) \mathcal{T}_{n(s) \nu}(T, \sigma)\right)=\mathcal{G}_{n(r) \mu ; n(s) \nu}(\sigma)
\end{gathered}
$$

which are well-known in ordinary (space-time) current-algebraic orbifold theory. Listed here in particular are the twisted structure constants $\mathcal{F}(\sigma)$, the twisted representation matrices $\mathcal{T}$ (which satisfy the orbifold Lie algebra $\hat{g}(\sigma)$ in $(2.14 \mathrm{e})$ ), and the (invertible) twisted data matrix $\mathcal{M}$ - where $\chi(\sigma)_{n(r) \mu}$ are arbitrary normalization constants. These duality transformations have been evaluated [3,5,14-17] for all basic orbifold types, except for a few outer-automorphic orbifolds of $\mathbb{Z}_{2}$ type. In the case of permutation-invariant systems

$$
g=\oplus \mathfrak{g}^{I}, \quad \mathfrak{g}^{I} \simeq \mathfrak{g}, \quad k_{I}=k, \quad T^{I} \simeq T
$$

one has $M(k, T) \propto \mathbb{1}$ and the twisted data matrix satisfies $\mathcal{M}(\mathcal{T}, \sigma)=M(k, T)$.

We are now in a position to define the eigengroup elements

$$
\begin{gathered}
\mathcal{G}(\mathcal{T}, \xi, \sigma) \equiv U U(T, \sigma) \tilde{g}(T, \xi) U^{\dagger}(T, \sigma) U^{\dagger}=\left(\begin{array}{cc}
\mathcal{G}_{(0)}(\mathcal{T}, \xi, \sigma) & \mathcal{G}_{(1)}(\mathcal{T}, \xi, \sigma) \\
\mathcal{G}_{(1)}(\mathcal{T}, \xi, \sigma) & \mathcal{G}_{(0)}(\mathcal{T}, \xi, \sigma)
\end{array}\right) \\
\mathcal{G}_{(u)}(\mathcal{T}, \xi, \sigma) \equiv \frac{1}{2} U(T, \sigma)\left(g(T, \xi, t)+(-1)^{u} g^{-1}(T,-\xi, t)\right) U^{\dagger}(T, \sigma), \quad \bar{u}=0,1
\end{gathered}
$$




$$
\begin{gathered}
\mathcal{G}^{-1}(\mathcal{T}, \xi, \sigma)=\tau_{3} \mathcal{G}(\mathcal{T},-\xi, \sigma) \tau_{3}=\left(\begin{array}{cc}
\mathcal{G}_{(0)}(\mathcal{T},-\xi, \sigma) & -\mathcal{G}_{(1)}(\mathcal{T},-\xi, \sigma) \\
-\mathcal{G}_{(1)}(\mathcal{T},-\xi, \sigma) & \mathcal{G}_{(0)}(\mathcal{T},-\xi, \sigma)
\end{array}\right) \\
{\left[\tau_{1}, \mathcal{G}(\mathcal{T}, \xi, \sigma)\right]=\left[\mathcal{M}(\mathcal{T}, \sigma) \otimes \mathbb{1}_{2}, \mathcal{G}(\mathcal{T}, \xi, \sigma)\right]=0} \\
U=U^{\dagger} \equiv \frac{1}{\sqrt{2}}\left(\begin{array}{cc}
1 & 1 \\
1 & -1
\end{array}\right), \quad \tau_{1} U^{\dagger}=U^{\dagger} \tau_{3}
\end{gathered}
$$

which (like the matrix group elements) are also two-component fields. The eigengroup elements are the high-level limits of the eigenprimary fields of Ref. [25]. Here $U^{\dagger}$ plays the same role in the two-dimensional space that $U^{\dagger}(T, \sigma)$ plays in the space of rep $T$, and the product $U^{\dagger}(T, \sigma) U^{\dagger}$ plays the role of the total $U^{\dagger}$ shown in Fig. 1. The form of the inverse in (2.16c) follows directly from Eq. (2.9d), but further non-local quadratic relations can be obtained by comparing this form with (2.16a).

The eigengroup elements are defined to diagonalize the action of the automorphism $\hat{h}_{\sigma}$ :

$$
\begin{gathered}
\mathcal{G}(\mathcal{T}, \xi, \sigma)^{\prime}=\tau_{3} E(T, \sigma) \mathcal{G}(\mathcal{T}, \xi, \sigma) E^{*}(T, \sigma) \tau_{3} \\
\mathcal{G}^{-1}(\mathcal{T}, \xi, \sigma)^{\prime}=\tau_{3} E(T, \sigma) \mathcal{G}^{-1}(\mathcal{T}, \xi, \sigma) E^{*}(T, \sigma) \tau_{3} \\
\mathcal{G}_{(u)}(\mathcal{T}, \xi, \sigma)^{\prime}=(-1)^{u} E(T, \sigma) \mathcal{G}_{(u)}(\mathcal{T}, \xi, \sigma) E^{*}(T, \sigma) .
\end{gathered}
$$

At the tangent-space level, one finds the corresponding structure

$$
\begin{gathered}
\mathcal{G}(\mathcal{T}(T, \sigma), \xi, \sigma)=e^{i \mathfrak{b}^{n(r) \mu u}(\xi, \sigma) \mathcal{T}_{n(r) \mu u}(T, \sigma)}, \quad \mathcal{T}_{n(r) \mu u}(T, \sigma) \equiv \mathcal{T}_{n(r) \mu}(T, \sigma) \tau_{u}, \quad u=0,1 \\
\tau_{0}=\mathbb{1}, \quad \vec{\tau}=\text { Pauli matrices } \\
\mathfrak{b}^{n(r) \mu u}(\xi, \sigma) \equiv \beta^{a \dot{I}}(\xi) \chi(\sigma)_{n(r) \mu}^{-1} U^{\dagger}(\sigma)_{a}{ }^{n(r) \mu}\left(\frac{1}{\sqrt{2}}\left(U^{\dagger}\right)_{\dot{I}}{ }^{u}\right) \\
=\frac{1}{2}\left(\beta^{a}(\xi)+(-1)^{u+1} \beta^{a}(-\xi)\right) \chi(\sigma)_{n(r) \mu}^{-1} U^{\dagger}(\sigma)_{a}^{n(r) \mu} \\
=\frac{1}{2}\left(\mathfrak{b}^{n(r) \mu}(\xi, \sigma)+(-1)^{u+1} \mathfrak{b}^{n(r) \mu}(-\xi, \sigma)\right) \\
\mathfrak{b}^{n(r) \mu}(\xi, \sigma) \equiv \beta^{a}(\xi) \chi(\sigma)_{n(r) \mu}^{-1} U^{\dagger}(\sigma)_{a}{ }^{n(r) \mu} \\
\mathfrak{b}^{n(r) \mu u}(-\xi, \sigma)=(-1)^{u+1} \mathfrak{b}^{n(r) \mu u}(\xi, \sigma) \\
\mathfrak{b}^{n(r) \mu u}(\xi, \sigma)^{\prime}=\mathfrak{b}^{n(r) \mu u}(\xi, \sigma) e^{2 \pi i\left(\frac{n(r)}{\rho(\sigma)}+\frac{u}{2}\right)}
\end{gathered}
$$

in terms of the tangent-space eigencoordinates $\mathfrak{b}$. The world-sheet parity (2.18f) of the tangent-space eigencoordinates implies the world-sheet parity (2.16c) of the eigengroup elements. With the $\mathcal{T}$-selection rule (2.14d), the (diagonal) automorphic response (2.18g) 
similarly implies the automorphic response (2.17a) of the eigengroup elements. We also note that the total twisted representation matrices in (2.18a) satisfy the total orbifold Lie algebra $\hat{g}_{O}(\sigma)$ of sector $\hat{h}_{\sigma}$

$$
\begin{array}{r}
{\left[\mathcal{T}_{n(r) \mu u}(T, \sigma), \mathcal{T}_{n(s) \nu v}(T, \sigma)\right]=i \mathcal{F}_{n(r) \mu ; n(s) \nu}{ }^{n(r)+n(s), \delta}(\sigma) \mathcal{T}_{n(r)+n(s), \delta, u+v}(T, \sigma)} \\
\widehat{\operatorname{Tr}}\left(\left(\mathcal{M} \otimes \mathbb{1}_{2}\right) \mathcal{T}_{n(r) \mu u}(T, \sigma) \mathcal{T}_{n(s) \nu v}(T, \sigma)\right)=\mathcal{G}_{n(r) \mu u ; n(s) \nu v}(\sigma) \\
\equiv 2 \delta_{u+v, 0 \bmod 2} \mathcal{G}_{n(r) \mu ; n(s) \nu}(\sigma)
\end{array}
$$

where $\mathcal{F}(\sigma)$ are the twisted structure constants defined in (2.14b) and $\mathcal{G}(\sigma)$ in (2.19b) is the total twisted metric of sector $\hat{h}_{\sigma}$.

\subsection{Group Orbifold Elements and Definite Monodromies}

We now invoke the principle of local isomorphisms [3, 5, 14] to go from the eigenfield formulation to twisted open-string sector $\hat{h}_{\sigma}$ of the orientation orbifold

$$
\mathcal{G}(\mathcal{T}, \xi, t, \sigma) \underset{\sigma}{\longrightarrow} \hat{g}(\mathcal{T}, \xi, t, \sigma), \quad \mathfrak{b}(\xi, t, \sigma) \underset{\sigma}{\longrightarrow} \hat{\beta}(\xi, t, \sigma)
$$

where $\hat{g}$ and $\hat{\beta}$ are respectively the group orbifold elements and the twisted tangent-space coordinates of sector $\hat{h}_{\sigma}$. Both of these twisted fields inherit the index structure and the constraints of the corresponding eigenfields

$$
\begin{gathered}
\hat{g}(\mathcal{T}, \xi, \sigma)=\left\{\hat{g}(\mathcal{T}, \xi, \sigma)_{N(r) \mu u}^{N(s) \nu v}\right\} \\
{\left[\mathcal{M} \otimes \mathbb{1}_{2}, \hat{g}(\mathcal{T}, \xi, \sigma)\right]=0} \\
{\left[\tau_{1}, \hat{g}(\mathcal{T}, \xi, \sigma)\right]=0} \\
\hat{g}(\mathcal{T},-\xi, \sigma)=\tau_{3} \hat{g}^{-1}(\mathcal{T}, \xi, \sigma) \tau_{3} \\
\hat{g}(\mathcal{T},-\xi, \sigma)_{N(r) \mu u}{ }^{N(s) \nu v}=(-1)^{u-v} \hat{g}^{-1}(\mathcal{T}, \xi, \sigma)_{N(r) \mu u}{ }^{N(s) \nu v} \\
\hat{g}(\mathcal{T}, \xi, \sigma)=e^{i \hat{\beta}^{n(r) \mu u}(\xi, \sigma) \mathcal{T}_{n(r) \mu u}(T, \sigma)} \\
\hat{\beta}^{n(r) \mu u}(-\xi, \sigma)=(-1)^{u+1} \hat{\beta}^{n(r) \mu u}(\xi, \sigma), \quad \bar{u}=0,1
\end{gathered}
$$

where Eq. (2.22a) is the twisted tangent-space form of the group orbifold element. The tangent-space form guarantees that the group orbifold elements form a group because the total matrices $\mathcal{T}$ satisfy the total orbifold Lie algebra $\hat{g}_{O}(\sigma)$. Moreover, Eqs. (2.22a) and (2.22b) together satisfy all the conditions on $\hat{g}$ in Eq. (2.21). Finally, the world-sheet parities 
(2.21d ) and (2.22b) tell us that the natural range of $\xi$ is the strip $0 \leq \xi \leq \pi$, and restriction to the strip is equivalent to the conventional orbifold identification $-\xi \sim \xi$.

The relations (2.21) also give the more explicit two-component structure of the group orbifold elements

$$
\begin{aligned}
\hat{g}(\mathcal{T}, \xi, \sigma)=\left(\begin{array}{cc}
\hat{g}_{(0)}(\mathcal{T}, \xi, \sigma) & \hat{g}_{(1)}(\mathcal{T}, \xi, \sigma) \\
\hat{g}_{(1)}(\mathcal{T}, \xi, \sigma) & \hat{g}_{(0)}(\mathcal{T}, \xi, \sigma)
\end{array}\right), \quad\left[\mathcal{M}, \hat{g}_{(u)}(\mathcal{T}, \xi, \sigma)\right]=0 \\
\hat{g}^{-1}(\mathcal{T}, \xi, \sigma)=\left(\begin{array}{cc}
\hat{g}_{(0)}(\mathcal{T},-\xi, \sigma) & -\hat{g}_{(1)}(\mathcal{T},-\xi, \sigma) \\
-\hat{g}_{(1)}(\mathcal{T},-\xi, \sigma) & \hat{g}_{(0)}(\mathcal{T},-\xi, \sigma)
\end{array}\right) \\
\hat{g}(\mathcal{T}, \xi, \sigma)_{N(r) \mu u}{ }^{N(s) \nu v}=\hat{g}_{(u-v)}(\mathcal{T}, \xi, \sigma)_{N(r) \mu} N(s) \nu \\
\hat{g}^{-1}(\mathcal{T}, \xi, \sigma)_{N(r) \mu u}{ }^{N(s) \nu v}=(-1)^{u-v} \hat{g}_{(u-v)}(\mathcal{T},-\xi, \sigma)
\end{aligned}
$$

and the form (2.23b) of the inverse implies the further non-local quadratic relations

$$
\begin{aligned}
& \hat{g}_{(0)}(\mathcal{T}, \xi, \sigma) \hat{g}_{(0)}(\mathcal{T},-\xi, \sigma)-\hat{g}_{(1)}(\mathcal{T}, \xi, \sigma) \hat{g}_{(1)}(\mathcal{T},-\xi, \sigma)=\mathbb{1} \\
& \hat{g}_{(0)}(\mathcal{T}, \xi, \sigma) \hat{g}_{(1)}(\mathcal{T},-\xi, \sigma)-\hat{g}_{(1)}(\mathcal{T}, \xi, \sigma) \hat{g}_{(0)}(\mathcal{T},-\xi, \sigma)=0
\end{aligned}
$$

among the reduced components $\hat{g}_{(u)}$ of the group orbifold elements.

In addition to these properties, one may attempt to impose definite monodromies on $\hat{g}$ and $\hat{\beta}$, as they would follow from Eqs. (2.17a), (2.18g) by a standard application of local isomorphisms

$$
\begin{gathered}
\text { automorphic responses of } \mathcal{G}, \mathfrak{b} \underset{\sigma}{\longrightarrow} \text { monodromies of } \hat{g}, \hat{\beta} \\
\hat{g}(\mathcal{T}(T, \sigma), \xi+2 \pi, \sigma)=\tau_{3} E(T, \sigma) \hat{g}(\mathcal{T}(T, \sigma), \xi, \sigma) E^{*}(T, \sigma) \tau_{3} \\
\hat{g}^{-1}(\mathcal{T}(T, \sigma), \xi+2 \pi, \sigma)=\tau_{3} E(T, \sigma) \hat{g}^{-1}(\mathcal{T}(T, \sigma), \xi, \sigma) E^{*}(T, \sigma) \tau_{3} \\
\hat{g}(\mathcal{T}, \xi+2 \pi, \sigma)_{N(r) \mu u}{ }^{N(s) \nu v}=e^{-2 \pi i\left(\frac{N(r)-N(s)}{R(\sigma)}+\frac{u-v}{2}\right)} \hat{g}(\mathcal{T}, \xi, \sigma)_{N(r) \mu u}{ }^{N(s) \nu v} \\
\hat{g}_{(u)}(\mathcal{T}(T, \sigma), \xi+2 \pi, \sigma)=(-1)^{u} E(T, \sigma) \hat{g}_{(u)}(\mathcal{T}(T, \sigma), \xi, \sigma) E^{*}(T, \sigma) \\
\hat{\beta}^{n(r) \mu u}(\xi+2 \pi, \sigma)=\hat{\beta}^{n(r) \mu u}(\xi, \sigma) e^{2 \pi i\left(\frac{n(r)}{\rho(\sigma)}+\frac{u}{2}\right)}
\end{gathered}
$$

where the eigenvalue matrix $E(T, \sigma)$ is defined in Eq. (2.11). As above, Eqs. (2.25f) and the $\mathcal{T}$-selection rule (2.14d $)$ imply Eq. (2.25b), and moreover, all these monodromies are consistent with each other. 
In Ref. 25] however, we argued from the twisted currents (see Subsec. 3.3) that the group orbifold elements in the open-string sectors of the WZW orientation orbifolds have the definite monodromy (2.25b) only in the case

$$
h_{\sigma}^{2}=1, \quad E^{2}(T, \sigma)=1, \quad \frac{\bar{n}(r)}{\rho(\sigma)} \in\left\{0, \frac{1}{2}\right\}
$$

and will have mixed monodromy when $h_{\sigma}^{2} \neq 1$.

We present here another version of this argument which shows that the definite monodromies in Eq. (2.25) are not consistent with the world-sheet parities (2.21d), (2.22b) beyond the case $h_{\sigma}^{2}=1$.

To find this inconsistency, consider generic orbits of the world-sheet parity and monodromy, following the steps:

$$
\begin{aligned}
\hat{g}(\mathcal{T}(T, \sigma), \xi, \sigma) & =\tau_{3} E(T, \sigma) \hat{g}(\mathcal{T}(T, \sigma), \xi-2 \pi, \sigma) E^{*}(T, \sigma) \tau_{3} \\
= & E(T, \sigma) \hat{g}^{-1}(\mathcal{T}(T, \sigma), 2 \pi-\xi, \sigma) E^{*}(T, \sigma) \\
= & \tau_{3} E^{2}(T, \sigma) \hat{g}^{-1}(\mathcal{T}(T, \sigma),-\xi, \sigma) E^{* 2}(T, \sigma) \tau_{3} \\
= & E^{2}(T, \sigma) \hat{g}(\mathcal{T}(T, \sigma), \xi, \sigma) E^{* 2}(T, \sigma) \\
\hat{\beta}^{n(r) \mu u}(\xi) & =\hat{\beta}^{n(r) \mu u}(\xi-2 \pi) e^{2 \pi i\left(\frac{n(r)}{\rho(\sigma)}+\frac{u}{2}\right)} \\
& =-\hat{\beta}^{n(r) \mu u}(2 \pi-\xi) e^{2 \pi i \frac{n(r)}{\rho(\sigma)}} \\
& =-\hat{\beta}^{n(r) \mu u}(-\xi) e^{4 \pi i \frac{n(r)}{\rho(\sigma)}}(-1)^{u} \\
& =\hat{\beta}^{n(r) \mu u}(\xi) e^{4 \pi i \frac{n(r)}{\rho(\sigma)}} .
\end{aligned}
$$

These relations are easily expressed as the selection rules

$$
\begin{gathered}
\hat{g}(\mathcal{T}(T, \sigma), \xi, \sigma)_{N(r) \mu u}^{N(s) \nu v}\left(1-e^{-4 \pi i\left(\frac{N(r)-N(s)}{R(\sigma)}\right)}\right)=0 \\
\Rightarrow \hat{g}(\mathcal{T}(T, \sigma), \xi, \sigma)_{N(r) \mu u}^{N(s) \nu v}=0 \text { unless } \frac{N(r)-N(s)}{R(\sigma)} \in \frac{1}{2} \mathbb{Z} \\
\hat{\beta}^{n(r) \mu u}(\xi, \sigma)\left(1-e^{4 \pi i \frac{n(r)}{\rho(\sigma)}}\right)=0 \Rightarrow \hat{\beta}^{n(r) \mu u}(\xi, \sigma)=0 \text { unless } \frac{n(r)}{\rho(\sigma)} \in \frac{1}{2} \mathbb{Z}
\end{gathered}
$$

which are again consistent with each other by the $\mathcal{T}$-selection rule (2.14d). The selection rules tell us however that the twisted fields with world-sheet parity and definite monodromy can have no support beyond the case $h_{\sigma}^{2}=1$ in Eq. (2.26).

This conclusion is a restriction [25] on that part of the principle of local isomorphisms which deals with the monodromies of open-string group orbifold elements, so that the 
correct form of Eq. (2.25a) for the open-string sectors of the orientation orbifolds is:

$$
h_{\sigma}^{2}=1: \quad \text { automorphic responses of } \mathcal{G}, \mathfrak{b} \underset{\sigma}{\longrightarrow} \text { monodromies of } \hat{g}, \hat{\beta} \text {. }
$$

We emphasize with Ref. [25] that all the other results obtained by eigenfields and local isomorphisms hold for all $\hat{h}_{\sigma}$ - except for the definite $2 \pi$-monodromy (2.25), which holds only for $h_{\sigma}^{2}=1$.

In spite of the absence of definite $2 \pi$-monodromy for $\hat{g}$ when $h_{\sigma}^{2} \neq 1$, each sector $\hat{h}_{\sigma}=$ $\tau_{1} \times h_{\sigma}$ is a twisted open string because each sector contains at least some fractionally-moded twisted currents (see Subsec. 3.4).

For completeness, we finally note that the class of $h_{\sigma}^{2}=1$ open-string sectors can be subdivided into two cases 25]

$$
\begin{aligned}
h_{\sigma}=1: & \rho(\sigma)=1, \quad \omega\left(h_{\sigma}\right)=1, \quad \bar{n}(r)=\bar{N}(r)=0 \\
h_{\sigma}^{2}=1, & h_{\sigma} \neq 1: \quad \rho(\sigma)=2, \quad \omega\left(h_{\sigma}\right)^{2}=1, \quad \bar{n}(r), \bar{N}(r) \in\{0,1\}
\end{aligned}
$$

where (2.30a a is the basic open-string sector, and (2.30b collects the generic open-string sectors with $h_{\sigma}^{2}=1$. We will return to further discuss the special properties of the class $h_{\sigma}^{2}=1$ in Subsec. 3.5.

\section{Actions and Boundary Conditions on the Solid Half Cylinder}

Our next task is to find the action which describes the open-string WZW orientationorbifold sectors above. In order to apply the method of eigenfields and the principle of local isomorphisms to this problem, we will first rewrite the standard WZW action in an unconventional but equivalent form on the solid half cylinder $\Gamma_{1 / 2}$ (see Fig. 22).

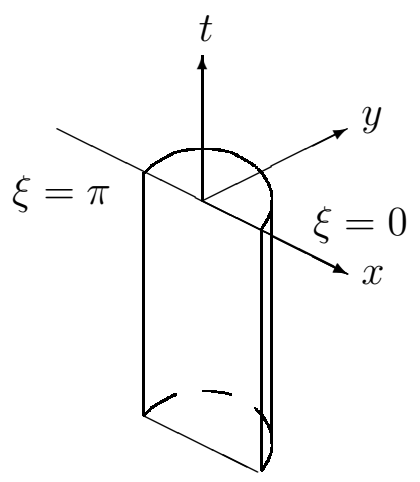

Fig.2, The Solid Half Cylinder 


\subsection{WZW Action on the Solid Half Cylinder}

The standard untwisted WZW action [29, 30] on the solid cylinder $\Gamma$ is easily generalized to semisimple $g$ [14]

$$
\begin{aligned}
S_{W Z W}[M, g ; \Gamma]= & -\frac{1}{8 \pi} \int d t \int_{0}^{2 \pi} d \xi \operatorname{Tr}\left(M\left(g^{-1}(T, \xi) \partial_{+} g(T, \xi) g^{-1}(T, \xi) \partial_{-} g(T, \xi)\right)\right) \\
& -\frac{1}{12 \pi} \int_{\Gamma} \operatorname{Tr}\left(M\left(g^{-1}(T, \xi) d g(T, \xi)\right)^{3}\right), \quad \partial_{ \pm}=\partial_{t} \pm \partial_{\xi}
\end{aligned}
$$

where the data matrix $M$ is defined in Eq. (2.4). As usual, the integration over $\Gamma$ in the

WZW term involves $\int d t \int_{0}^{2 \pi} d \xi \int d \rho$, where $\rho$ is the radial coordinate. Using the properties of $M$ in Eqs. (2.5) and (2.11), cyclicity of $T r$, and $2 \pi$-periodicity in the form

$$
f(\xi+2 \pi)=f(\xi) \Rightarrow \int_{0}^{2 \pi} d \xi f(-\xi)=\int_{0}^{2 \pi} d \xi f(\xi)
$$

it is then straightforward to check that the WZW action is invariant

$$
S_{W Z W}\left[M, g^{\prime} ; \Gamma\right]=S_{W Z W}[M, g ; \Gamma]
$$

under the general orientation-reversing automorphism in Eq. (2.8).

The WZW action can in fact be reexpressed in terms of the matrix group element $\tilde{g}$ on the solid half cylinder $\Gamma_{1 / 2}$

$$
\begin{aligned}
& S_{W Z W}\left[M \otimes \mathbb{1}_{2}, \tilde{g} ; \Gamma_{\frac{1}{2}}\right] \\
& \equiv-\frac{1}{8 \pi} \int d t \int_{0}^{\pi} d \xi \widehat{\operatorname{Tr}}\left(\left(M \otimes \mathbb{1}_{2}\right)\left(\tilde{g}^{-1}(T, \xi) \partial_{+} \tilde{g}(T, \xi) \tilde{g}^{-1}(T, \xi) \partial_{-} \tilde{g}(T, \xi)\right)\right) \\
& \quad-\frac{1}{12 \pi} \int_{\Gamma_{\frac{1}{2}}} \widehat{\operatorname{Tr}}\left(\left(M \otimes \mathbb{1}_{2}\right)\left(\tilde{g}^{-1}(T, \xi) d \tilde{g}(T, \xi)\right)^{3}\right)=S_{W Z W}[M, g ; \Gamma]
\end{aligned}
$$

where integration over $\Gamma_{1 / 2}$ involves $\int d t \int_{0}^{\pi} d \xi \int d \rho$. To see this equality for the WZW term in particular, follow the steps

$$
\begin{gathered}
\gamma(t, \xi, \rho) \equiv \epsilon^{A B C} \operatorname{Tr}\left(M g^{-1}(T, \xi) \partial_{A} g(T, \xi) g^{-1}(T, \xi) \partial_{B} g(T, \xi) g^{-1}(T, \xi) \partial_{C} g(T, \xi)\right) \\
\{A, B, C\}=\{t, \xi, \rho\}, \quad \epsilon^{t \xi \rho}=1
\end{gathered}
$$




$$
\begin{aligned}
\int_{\Gamma} \operatorname{Tr}\left(M\left(g^{-1}(T, \xi) d g(T, \xi)\right)^{3}\right) & =\int d t \int_{0}^{2 \pi} d \xi \int d \rho \gamma(t, \xi, \rho) \\
& =\int d t \int_{0}^{\pi} d \xi \int d \rho(\gamma(t, \xi, \rho)+\gamma(t,-\xi, \rho)) \\
& =\int_{\Gamma_{\frac{1}{2}}} \widehat{\operatorname{Tr}}\left(\left(M \otimes \mathbb{1}_{2}\right)\left(\tilde{g}^{-1}(T, \xi) d \tilde{g}(T, \xi)\right)^{3}\right)
\end{aligned}
$$

where $2 \pi$-periodicity of the quantity $\gamma(t, \xi, \rho)$ was used to obtain (3.5c). For the last equality, the reader may find it helpful to use the form (2.9a) of the matrix group element to write out (3.5d) as a sum of two traces $\operatorname{Tr}$ and use cyclicity. A similar argument suffices to rewrite the kinetic term of the WZW action as

$$
-\frac{1}{8 \pi} \int d t \int_{0}^{\pi} d \xi\left[\operatorname{Tr}\left(M\left(g^{-1}(T, \xi) \partial_{+} g(T, \xi) g^{-1}(T, \xi) \partial_{-} g(T, \xi)\right)\right)+(\xi \rightarrow-\xi)\right]
$$

which sums to the $\tilde{g}$ form of the kinetic term in Eq. (3.4).

The $\Gamma_{1 / 2}$ form (3.4) of the WZW action is transparently invariant under the twocomponent form (2.9) of the general orientation-reversing automorphism, this time without use of periodicity. For reference below, we also note that both integrands in the action (3.4) are even under world-sheet parity $\xi \leftrightarrow-\xi$.

The next step in the orbifold program is to express the $\Gamma_{1 / 2}$ form (3.4) of the action in terms of the eigengroup elements

$$
\begin{aligned}
S_{W Z W}\left[\mathcal{M} \otimes \mathbb{1}_{2}, \mathcal{G} ; \Gamma_{\frac{1}{2}}\right] \\
\equiv-\frac{1}{8 \pi} \int d t \int_{0}^{\pi} d \xi \widehat{\operatorname{Tr}}\left(\left(\mathcal{M} \otimes \mathbb{1}_{2}\right)\left(\mathcal{G}^{-1}(\mathcal{T}, \xi) \partial_{+} \mathcal{G}(\mathcal{T}, \xi) \mathcal{G}^{-1}(\mathcal{T}, \xi) \partial_{-} \mathcal{G}(\mathcal{T}, \xi)\right)\right) \\
\quad-\frac{1}{12 \pi} \int_{\Gamma_{\frac{1}{2}}} \widehat{\operatorname{Tr}}\left(\left(\mathcal{M} \otimes \mathbb{1}_{2}\right)\left(\mathcal{G}^{-1}(\mathcal{T}, \xi) d \mathcal{G}(\mathcal{T}, \xi)\right)^{3}\right)=S_{W Z W}\left[M \otimes \mathbb{1}_{2}, \tilde{g}(\mathcal{G}) ; \Gamma_{\frac{1}{2}}\right]
\end{aligned}
$$

where the eigengroup element $\mathcal{G}$ and the twisted data matrix $\mathcal{M}$ are defined in Eqs. (2.16a) and (2.14f) respectively. Using the properties of $\mathcal{M}$ in Eq. (2.14g), this form of the WZW action is invariant under the diagonal action (2.17) of the orientation-reversing automorphism. 


\subsection{The WZW Orientation-Orbifold Action}

We are now prepared to apply the principle of local isomorphisms to the eigengroup elements $\mathcal{G}$ in the context of the half-cylinder WZW action (3.7)

$$
\begin{gathered}
\mathcal{G}(\mathcal{T}, \xi, t, \sigma) \underset{\sigma}{\longrightarrow} \hat{g}(\mathcal{T}, \xi, t, \sigma) \\
S_{W Z W}\left[\mathcal{M} \otimes \mathbb{1}_{2}, \mathcal{G} ; \Gamma_{\frac{1}{2}}\right] \underset{\sigma}{\longrightarrow} \hat{S}_{\hat{g}_{O}(\sigma)}\left[\mathcal{M} \otimes \mathbb{1}_{2}, \hat{g} ; \Gamma_{\frac{1}{2}}\right]
\end{gathered}
$$

where $\hat{g}(\mathcal{T}, \xi, t, \sigma)$ are the group orbifold elements of Sec. 2. This gives the $W Z W$ orientationorbifold action of open-string sector $\hat{h}_{\sigma}$ on the solid half cylinder $\Gamma_{1 / 2}$

$$
\begin{gathered}
\hat{S}_{\hat{g}_{O}(\sigma)}\left[\mathcal{M} \otimes \mathbb{1}_{2}, \hat{g} ; \Gamma_{\frac{1}{2}}\right] \equiv \\
-\frac{1}{8 \pi} \int d t \int_{0}^{\pi} d \xi \widehat{\operatorname{Tr}}\left(\left(\mathcal{M} \otimes \mathbb{1}_{2}\right)\left(\hat{g}^{-1}(\mathcal{T}, \xi) \partial_{+} \hat{g}(\mathcal{T}, \xi) \hat{g}^{-1}(\mathcal{T}, \xi) \partial_{-} \hat{g}(\mathcal{T}, \xi)\right)\right) \\
\quad-\frac{1}{12 \pi} \int_{\Gamma_{\frac{1}{2}}} \widehat{\operatorname{Tr}}\left(\left(\mathcal{M} \otimes \mathbb{1}_{2}\right)\left(\hat{g}^{-1}(\mathcal{T}, \xi) d \hat{g}(\mathcal{T}, \xi)\right)^{3}\right) \\
\hat{g}(\mathcal{T},-\xi, \sigma)=\tau_{3} \hat{g}^{-1}(\mathcal{T}, \xi, \sigma) \tau_{3} \\
{\left[\tau_{1}, \hat{g}(\mathcal{T}(T, \sigma), \xi, \sigma)\right]=\left[\mathcal{M} \otimes \mathbb{1}_{2}, \hat{g}(\mathcal{T}(T, \sigma), \xi, \sigma)\right]=0}
\end{gathered}
$$

where the second term in Eq. (3.9a) is the orientation-orbifold WZW term. Here we have followed the convention of the orbifold program [14, 15, 23, 24, in labelling the orientationorbifold action of sector $\hat{h}_{\sigma}$ by its associated orbifold Lie algebra $\hat{g}_{O}(\sigma)$ in (2.19a). It is not difficult to check with Eq. (3.9b) that each integrand in this action is symmetric under world-sheet parity $\xi \leftrightarrow-\xi$.

Although the WZW action (3.4) and the WZW orientation-orbifold action (3.9) are both defined on the solid half cylinder $\Gamma_{1 / 2}$, only the latter truly describes an open string. The fundamental reason for this is that the orientation-orbifold sector has only a single untwisted Virasoro algebra [25, which we will review at the classical level in Subsec. 3.4.

The WZW orientation-orbifold action (3.9), which describes all open-string WZW orientation-orbifold sectors, is a central result of this paper. Closed-string sectors of the WZW orientation orbifolds are described by the standard WZW orbifold action [14.

\subsection{Variation of the Half-Cylinder Actions}

In this subsection, we study the variation (with special attention to boundaries) of the halfcylinder form of the WZW action (3.4) and the WZW orientation-orbifold action (3.9). 
For brevity, we will study only the WZW orientation-orbifold action explicitly, but the same steps can be followed for the simpler case of the WZW action by the substitution $\mathcal{M} \rightarrow M, \hat{g} \rightarrow \tilde{g}$. For this discussion, we will need the general variations

$$
\begin{aligned}
& \widehat{\operatorname{Tr}}\left(\left(\mathcal{M} \otimes \mathbb{1}_{2}\right) \hat{g}^{-1} \partial_{+} \hat{g} \hat{g}^{-1} \partial_{-} \hat{g}\right) \\
& =-\widehat{\operatorname{Tr}}\left[\left(\mathcal{M} \otimes \mathbb{1}_{2}\right)\left(\partial_{-}\left(\hat{g}^{-1} \partial_{+} \hat{g}\right)+\partial_{+}\left(\hat{g}^{-1} \partial_{-} \hat{g}\right)\right) \hat{g}^{-1} \delta \hat{g}\right] \\
& +2 \widehat{\operatorname{Tr}}\left[\left(\mathcal{M} \otimes \mathbb{1}_{2}\right)\left(\partial_{t}\left(\hat{g}^{-1} \partial_{t} \hat{g} \hat{g}^{-1} \delta \hat{g}\right)-\partial_{\xi}\left(\hat{g}^{-1} \partial_{\xi} \hat{g} \hat{g}^{-1} \delta \hat{g}\right)\right)\right] \\
& \epsilon^{A B C} \delta \widehat{\operatorname{Tr}}\left(\left(\mathcal{M} \otimes \mathbb{1}_{2}\right) \hat{g}^{-1} \partial_{A} \hat{g} \hat{g}^{-1} \partial_{B} \hat{g} \hat{g}^{-1} \partial_{C} \hat{g}\right)=\partial_{A} \hat{R}^{A} \\
& \hat{R}^{A} \equiv 3 \epsilon^{A B C} \widehat{\operatorname{Tr}}\left(\left(\mathcal{M} \otimes \mathbb{1}_{2}\right) \hat{g}^{-1} \partial_{B} \hat{g} \hat{g}^{-1} \partial_{C} \hat{g} \hat{g}^{-1} \delta \hat{g}\right) \\
& \hat{R}^{\rho}=\frac{3}{2} \widehat{\operatorname{Tr}}\left[\left(\mathcal{M} \otimes \mathbb{1}_{2}\right)\left(\partial_{-}\left(\hat{g}^{-1} \partial_{+} \hat{g}\right)-\partial_{+}\left(\hat{g}^{-1} \partial_{-} \hat{g}\right)\right)\right]
\end{aligned}
$$

which are standard in WZW theory, except that we have kept the total derivative terms in Eq. (3.10a).

Using the world-sheet parity (3.9b) and cyclicity of $\widehat{T r}$, we can now verify the following behavior under world-sheet parity

$$
\begin{aligned}
\hat{K}(\xi) \equiv & \widehat{\operatorname{Tr}}\left(\left(\mathcal{M} \otimes \mathbb{1}_{2}\right) \hat{g}^{-1}(\xi) \partial_{\xi} \hat{g}(\xi) \hat{g}^{-1}(\xi) \delta \hat{g}(\xi)\right) \\
= & \widehat{\operatorname{Tr}}\left(\left(\mathcal{M} \otimes \mathbb{1}_{2}\right) \hat{g}(-\xi) \partial_{\xi} \hat{g}^{-1}(-\xi) \hat{g}(-\xi) \delta \hat{g}^{-1}(-\xi)\right) \\
= & \widehat{\operatorname{Tr}}\left(\left(\mathcal{M} \otimes \mathbb{1}_{2}\right) \hat{g}^{-1}(-\xi) \partial_{\xi} \hat{g}(-\xi) \hat{g}^{-1}(-\xi) \delta \hat{g}(-\xi)\right) \\
= & -\hat{K}(-\xi) \\
& \hat{R}^{t, \rho}(\xi)=\hat{R}^{t, \rho}(-\xi), \quad \hat{R}^{\xi}(\xi)=-\hat{R}^{\xi}(-\xi)
\end{aligned}
$$

where $\hat{K}(\xi)$ appears in the variation (3.10a of the kinetic term, and $\hat{R}(\xi)$ is defined in Eq. (3.10c).

The variation of the WZW orientation-orbifold action now proceeds as usual, requiring that the bulk and boundary terms cancel independently.

The boundary contribution of the total derivative term in Eq. (3.10a) to the variation of the kinetic term in the action (3.9) is

$$
\begin{aligned}
\frac{1}{4 \pi} \int d t \int_{0}^{\pi} d \xi \widehat{\operatorname{Tr}}\left(\partial_{\xi}\left(\hat{g}^{-1} \partial_{\xi} \hat{g} \hat{g}^{-1} \delta \hat{g}\right)\right) & =\frac{1}{4 \pi} \int d t(\hat{K}(\pi)-\hat{K}(0)) \equiv 0 \\
\rightarrow \hat{K}(\pi) & =\hat{K}(0)=0
\end{aligned}
$$


where Eq. (3.12b) is the local form of the variational boundary condition. The boundary condition at $\xi=0$ can also be obtained immediately from the world-sheet parity (3.11b).

For the variation of the orientation-orbifold $W Z W$ term in (3.9), it is convenient to introduce the standard Cartesian coordinates $(x, y, t)$ shown in Fig. 2. In these coordinates, the flat side of $\Gamma_{1 / 2}$ is the plane $y=0$, which is composed of the two half-planes at $\xi=0$ and at $\xi=\pi$. Then the boundary contribution from the flat side of $\Gamma_{1 / 2}$ is

$$
\begin{gathered}
\left.\vec{e}_{y}\right|_{\xi=0}=\vec{e}_{\xi},\left.\quad \vec{e}_{y}\right|_{\xi=\pi}=-\vec{e}_{\xi} \\
\int_{\Gamma_{\frac{1}{2}}} \partial_{A} \hat{R}^{A}=\int_{\partial \Gamma_{\frac{1}{2}}} d \vec{S} \cdot \overrightarrow{\hat{R}}=\int d t \int_{0}^{\pi} d \xi \hat{R}^{\rho}+\int_{y=0} d t d x\left(-\vec{e}_{y} \cdot \overrightarrow{\hat{R}}\right) \\
\int_{y=0} d t d x\left(-\vec{e}_{y} \cdot \overrightarrow{\hat{R}}\right)=\int_{y=0} d t d x\left(\hat{R}^{\xi}(\pi)-\hat{R}^{\xi}(0)\right) \equiv 0 \\
\rightarrow \hat{R}^{\xi}(\pi)=\hat{R}^{\xi}(0)=0
\end{gathered}
$$

where $\vec{e}_{i}$ denotes the unit vector in the $i$ direction. At $\xi=0$, the local form of this variational boundary condition also follows immediately from the world-sheet parity (3.11C).

The local forms of the boundary conditions in Eqs. (3.12b) and (3.13d) are important parts of the description of the WZW orientation-orbifold branes, on which the twisted open WZW strings end (see also Subsecs. 3.4 and 3.5).

Having dealt with the boundary terms, this leaves only the total bulk contribution

$$
\delta \hat{S}_{\hat{g}_{O}(\sigma)}\left[\mathcal{M} \otimes \mathbb{1}_{2}, \hat{g} ; \Gamma_{\frac{1}{2}}\right]=\frac{1}{4 \pi} \int d t \int_{0}^{\pi} d \xi \widehat{\operatorname{Tr}}\left(\left(\mathcal{M} \otimes \mathbb{1}_{2}\right) \partial_{-}\left(\hat{g}^{-1} \partial_{+} \hat{g}\right) \hat{g}^{-1} \delta \hat{g}\right)
$$

to the variation of the WZW orientation-orbifold action. Then, because the invertible matrix $\mathcal{M}$ commutes with the group orbifold elements, we find the open-string equations of motion

$$
\begin{array}{r}
\partial_{-} \hat{J}(\mathcal{T}, \xi, t)=0, \quad \hat{J}(\mathcal{T}, \xi, t) \equiv-\frac{i}{2} \hat{g}^{-1}(\mathcal{T}, \xi, t) \partial_{+} \hat{g}(\mathcal{T}, \xi, t) \\
\longrightarrow \partial_{+} \hat{\bar{J}}(\mathcal{T}, \xi, t)=0 \\
\hat{\bar{J}}(\mathcal{T}, \xi, t) \equiv-\frac{i}{2} \hat{g}(\mathcal{T}, \xi, t) \partial_{-} \hat{g}^{-1}(\mathcal{T}, \xi, t)=\tau_{3} \hat{J}(\mathcal{T},-\xi, t) \tau_{3} \\
{\left[\mathcal{M} \otimes \mathbb{1}_{2}, \hat{J}(\mathcal{T})\right]=\left[\mathcal{M} \otimes \mathbb{1}_{2}, \hat{\bar{J}}(\mathcal{T})\right]=\left[\tau_{1}, \hat{J}(\mathcal{T})\right]=\left[\tau_{1}, \hat{\bar{J}}(\mathcal{T})\right]=0}
\end{array}
$$

where $\hat{J}(\mathcal{T}), \hat{\bar{J}}(\mathcal{T})$ are the twisted open-string matrix currents. As indicated in (3.15b), $\hat{\bar{J}}(\mathcal{T})$ conservation follows from the conservation of $\hat{J}(\mathcal{T})$. The world-sheet parity relation 
(3.15c) between $\hat{J}$ and $\hat{\bar{J}}$ follows from Eq. (2.21d $)$, and this relation tells us that the twisted currents share the same set of twisted current modes (see the following subsection), as is appropriate for open strings. The twisted matrix currents are also two-component fields, and we find in particular

$$
\begin{gathered}
\hat{J}(\mathcal{T}, \xi, t)=\left(\begin{array}{cc}
\hat{J}_{(0)}(\mathcal{T}, \xi, t) & \hat{J}_{(1)}(\mathcal{T}, \xi, t) \\
\hat{J}_{(1)}(\mathcal{T}, \xi, t) & \hat{J}_{(0)}(\mathcal{T}, \xi, t)
\end{array}\right) \\
\hat{\bar{J}}(\mathcal{T}, \xi, t)=\left(\begin{array}{cc}
\hat{J}_{(0)}(\mathcal{T},-\xi, t) & -\hat{J}_{(1)}(\mathcal{T},-\xi, t) \\
-\hat{J}_{(1)}(\mathcal{T},-\xi, t) & \hat{J}_{(0)}(\mathcal{T},-\xi, t)
\end{array}\right) \\
\hat{J}_{(u)}(\mathcal{T}, \xi, t) \equiv-\frac{i}{2} \sum_{v=0}^{1}(-1)^{v} \hat{g}_{(v)}(\mathcal{T},-\xi, t) \partial_{+} \hat{g}_{(u-v)}(\mathcal{T}, \xi, t), \quad \bar{u}=0,1 .
\end{gathered}
$$

where the reduced components $\hat{g}_{(u)}$ of the group orbifold elements were defined in Eq. (2.23).

Returning to the simpler case of the $\Gamma_{1 / 2}$ form (3.4) of the untwisted WZW action, we find that the bulk contributions to the variation give the classical equation of motion

$$
\partial_{-}\left(\tilde{g}^{-1}(T, \xi, t) \partial_{+} \tilde{g}(T, \xi, t)\right)=0
$$

which implies the conservation of the usual untwisted left- and right-mover WZW currents. We can also explicitly verify the required local boundary conditions

$$
K(\pi)=K(0)=0, \quad R^{\xi}(\pi)=R^{\xi}(0)=0
$$

from the world-sheet parities

$$
K(\xi)=-K(-\xi), \quad R^{t, \rho}(\xi)=R^{t, \rho}(-\xi), \quad R^{\xi}(\xi)=-R^{\xi}(-\xi)
$$

and the $2 \pi$-periodicity of the untwisted fields. Here the quantities $K, R$ are obtained from those in Eqs. (3.10), (3.11) by the substitution $\mathcal{M} \rightarrow M$ and $\hat{g} \rightarrow \tilde{g}$.

The variational boundary conditions (3.12b), (3.13d) can also be explicitly verified from the monodromies in the case $h_{\sigma}^{2}=1$ (see Subsec. 3.5 ).

\subsection{More about the Twisted Open-String Currents}

In this subsection, we further discuss the classical forms of the twisted open-string currents and their corresponding twisted open-string stress tensors.

We begin this discussion by noting that the functional form of the twisted currents in $(3.15 \mathrm{a}, \mathrm{c})$ can be rewritten as the classical equations of motion of the group orbifold 
elements:

$$
\begin{gathered}
\partial_{+} \hat{g}(\mathcal{T}, \xi, t)=2 i \hat{g}(\mathcal{T}, \xi, t) \hat{J}(\mathcal{T}, \xi, t), \quad \partial_{-} \hat{g}(\mathcal{T}, \xi, t)=-2 i \hat{\bar{J}}(\mathcal{T}, \xi, t) \hat{g}(\mathcal{T}, \xi, t) \\
\hat{J}(\mathcal{T}, \xi, t)=\hat{J}_{n(r) \mu u}(\xi, t) \mathcal{G}^{n(r) \mu u ; n(s) \nu v}(\sigma) \mathcal{T}_{n(s) \nu} \tau_{v} \\
\hat{\bar{J}}(\mathcal{T}, \xi, t)=\hat{J}_{n(r) \mu u}(-\xi, t) \mathcal{G}^{n(r) \mu u ; n(s) \nu v}(\sigma) \mathcal{T}_{n(s) \nu}(-1)^{v} \tau_{v} \\
\mathcal{G}^{n(r) \mu u ; n(s) \nu v}(\sigma)=\frac{1}{2} \delta_{u+v, 0 \bmod 2{ }_{2} \mathcal{G}^{n(r) \mu ; n(s) \nu}(\sigma)} \\
\mathcal{G}_{n(r) \mu ; n(t) \delta}(\sigma) \mathcal{G}^{n(t) \delta ; n(s) \nu}(\sigma)=\delta_{\mu}{ }^{\nu} \delta_{n(r)-n(s), 0 \bmod \rho(\sigma)} .
\end{gathered}
$$

In this system, the index-current expansions $(3.20 \mathrm{~b}, \mathrm{c})$ of each matrix current are taken from the high-level limit of the corresponding twisted vertex operator equations in Eq. (6.1) of Ref. [25]. As expected, the index-current expansions of $\hat{J}$ and $\hat{\bar{J}}$ satisfy the world-sheet parity relation in Eq. (3.15c).

For completeness, we also read off from Ref. [25] the bracket form of some important algebraic relations

$$
\begin{gathered}
\hat{J}_{n(r) \mu u}(\xi, t, \sigma)=\sum_{m} \hat{J}_{n(r) \mu u}\left(m+\frac{n(r)}{\rho(\sigma)}+\frac{u}{2}\right) e^{-i\left(m+\frac{n(r)}{\rho(\sigma)}+\frac{u}{2}\right)(t+\xi)}, \quad \bar{u}=0,1 \\
\hat{J}_{n(r) \mu u}(\xi+2 \pi, t, \sigma)=e^{-2 \pi i\left(\frac{n(r)}{\rho(\sigma)}+\frac{u}{2}\right)} \hat{J}_{n(r) \mu u}(\xi, t, \sigma) \\
\left\{\hat{J}_{n(r) \mu u}\left(m+\frac{n(r)}{\rho(\sigma)}+\frac{u}{2}\right), \hat{J}_{n(s) \nu v}\left(n+\frac{n(s)}{\rho(\sigma)}+\frac{v}{2}\right)\right\} \\
\left.=i \mathcal{F}_{n(r) \mu ; n(s) \nu}\right) \\
+\left(m+\frac{n(r)+n(s), \delta}{\rho(\sigma)}+\frac{u}{2}\right) \delta_{m+n+\frac{n(r)+n(s)}{\rho(\sigma)}+\frac{u+v}{2}, 0}\left(2 \delta_{u+v, 0 \bmod 2} \mathcal{G}_{n(r) \mu ; n(s) \nu}(\sigma)\right) \\
\left\{\hat{J}_{n(r) \mu u}\left(m+\frac{n(r)}{\rho(\sigma)}+\frac{u}{2}\right), \hat{g}(\mathcal{T}(T), \xi, t)\right\}=\hat{g}(\mathcal{T}(T), \xi, t)\left(\mathcal{T}_{n(r) \mu}(T) \tau_{u}\right) e^{i\left(m+\frac{n(r)}{\rho(\sigma)}+\frac{u}{2}\right)(t+\xi)} \\
-\left(\mathcal{T}_{n(r) \mu}(T)(-1)^{u} \tau_{u}\right) \hat{g}(\mathcal{T}(T), \xi, t) e^{i\left(m+\frac{n(r)}{\rho(\sigma)}+\frac{u}{2}\right)(t-\xi)}
\end{gathered}
$$

where the mode expansion (3.21a) and the monodromy (3.21b) of the currents are valid for all $\hat{h}_{\sigma}$, without restriction to $h_{\sigma}^{2}=1$. Note that the open-string twisted current algebra $\hat{\mathfrak{g}}_{O}(\sigma)$ in (3.21C) shares the same twisted structure constants $\mathcal{F}(\sigma)$ with the orbifold Lie algebra $\hat{g}_{O}(\sigma)$ in (2.19a), and that as expected for open strings, the twisted currents act simultaneously as left- and right-movers in Eq. (3.21d). Moreover, because $\bar{u}$ takes the values 0 and 1 , each open-string sector $\hat{h}_{\sigma}$ contains fractionally-moded currents.

We also note the monodromy of the twisted open-string matrix currents [25]

$$
\begin{aligned}
& \hat{J}(\mathcal{T}(T, \sigma), \xi+2 \pi, t)=\tau_{3} E(T, \sigma) \hat{J}(\mathcal{T}(T, \sigma), \xi, t) E^{*}(T, \sigma) \tau_{3} \\
& \hat{\bar{J}}(\mathcal{T}(T, \sigma), \xi+2 \pi, t)=\tau_{3} E^{*}(T, \sigma) \hat{\bar{J}}(\mathcal{T}(T, \sigma), \xi, t) E(T, \sigma) \tau_{3}
\end{aligned}
$$


which follow (by the $\mathcal{T}$-selection rule $(2.14 \mathrm{~d})$ ) from the monodromy $(3.21 \mathrm{~b})$ of the index currents - and hence are also true for all $\hat{h}_{\sigma}$.

With Ref. [25], we emphasize that the definite but clashing monodromies (3.22) of the twisted open-string matrix currents are precisely the reason that the group orbifold elements $\hat{g}$ cannot have definite monodromy beyond $h_{\sigma}^{2}=1$.

Using the general monodromies (3.22) and the world-sheet parity (3.15c), we may now give the boundary conditions of the twisted currents on the strip:

$$
\begin{gathered}
\hat{\bar{J}}(\mathcal{T}(T), 0)=\tau_{3} \hat{J}(\mathcal{T}(T), 0) \tau_{3} \\
\hat{\bar{J}}(\mathcal{T}(T), \pi)=E^{*}(T, \sigma) \hat{J}(\mathcal{T}(T), \pi) E(T, \sigma)
\end{gathered}
$$

In addition to the variational boundary conditions of the previous subsection, these twisted current boundary conditions should be included as another important part of the description of the orientation-orbifold branes in open-string sector $\hat{h}_{\sigma}$.

As promised, we finally mention the classical form of the twisted open-string stress tensors

$$
\begin{gathered}
\hat{\Theta}_{u}(\xi, t, \sigma)=\frac{1}{8 \pi} \mathcal{G}^{n(r) \mu ; n(s) \nu}(\sigma) \sum_{v=0}^{1} \hat{J}_{n(r) \mu v}(\xi, t, \sigma) \hat{J}_{n(s) \nu, u-v}(\xi, t, \sigma), \quad \bar{u}=0,1 \\
\partial_{-} \hat{\Theta}_{u}(\xi, t, \sigma)=0 \\
\hat{\Theta}_{u}(\xi+2 \pi, t, \sigma)=(-1)^{u} \hat{\Theta}_{u}(\xi, t, \sigma) \\
\hat{\Theta}_{u}(\xi, t, \sigma)=\frac{1}{2 \pi} \sum_{m} \hat{L}_{u}\left(m+\frac{u}{2}\right) e^{-i\left(m+\frac{u}{2}\right)(t+\xi)} \\
\left\{\hat{L}_{u}\left(m+\frac{u}{2}\right), \hat{L}_{v}\left(n+\frac{v}{2}\right)\right\}=\left(m-n+\frac{u-v}{2}\right) \hat{L}_{u+v}\left(m+n+\frac{u+v}{2}\right)
\end{gathered}
$$

where Eq. (3.24a) is the classical (high-level) limit of the twisted operator stress tensors of Ref. [25]. The unique physical stress tensor of sector $\hat{h}_{\sigma}$ is the component $\hat{\Theta}_{0}(\xi, t, \sigma)$ with trivial monodromy. Following Ref. 25], we emphasize that the classical form (3.24e) of the orbifold Virasoro algebra ${ }^{\ddagger 3}$ correspondingly contains only the single classical untwisted Virasoro subalgebra generated by $\left\{\hat{L}_{0}(m)\right\}$ - which tells us that sector $\hat{h}_{\sigma}$ is a twisted open string.

\footnotetext{
${ }^{\ddagger 3}$ The operator form of the orbifold Virasoro algebra is given in Ref. [25], where we discuss the quantum doubling $\hat{c}=2 c$ of the closed-string central charge $c$.
} 


\subsection{More about the Branes when $h_{\sigma}^{2}=1$}

In our discussion above, we have given two important parts of the description of the WZW orientation-orbifold branes for all $\hat{h}_{\sigma}$, namely the variational boundary conditions in Subsec. 3.3 and the twisted current boundary conditions in Subsec. 3.4. In what follows, we consider further structure of the branes, concentrating primarily but not exclusively on the case $h_{\sigma}^{2}=1$.

For the case $h_{\sigma}^{2}=1$, the monodromies in (2.25) give us a simple way to determine the boundary conditions of the twisted fields $\hat{g}, \hat{\beta}$ on the strip $0 \leq \xi \leq \pi$. As in Subsec. 2.3, we consider the combined action of the world-sheet parity (2.21d) and the monodromy (2.25b) of the group orbifold elements, this time for the special orbits associated to $\xi=0$ and $\pi$. This gives the boundary conditions on the group orbifold elements

$$
\begin{gathered}
\hat{g}^{-1}(\mathcal{T}(T, \sigma), 0, \sigma)=\tau_{3} \hat{g}(\mathcal{T}(T, \sigma), 0, \sigma) \tau_{3} \\
h_{\sigma}^{2}=1: \quad \hat{g}^{-1}(\mathcal{T}(T, \sigma), \pi, \sigma)=E(T, \sigma) \hat{g}(\mathcal{T}(T, \sigma), \pi, \sigma) E^{*}(T, \sigma)
\end{gathered}
$$

which describe further structure of the branes at the ends of each twisted open string. The boundary condition (3.25a at $\xi=0$ follows immediately from the world-sheet parity, and hence holds for all $\hat{h}_{\sigma}$, while the following steps for $h_{\sigma}^{2}=1$

$$
\hat{g}^{-1}(\mathcal{T}(T), \pi)=\tau_{3} E(T) \hat{g}^{-1}(\mathcal{T}(T),-\pi) E^{*}(T) \tau_{3}=E(T) \hat{g}(\mathcal{T}(T), \pi) E^{*}(T)
$$

were used to obtain the boundary condition at $\xi=\pi$.

Moreover, the $\tau_{1}$-constraint (2.21c) holds for all $\hat{h}_{\sigma}$ in the bulk and at the boundary

$$
\left[\tau_{1}, \hat{g}(\mathcal{T}(T, \sigma), 0, \sigma)\right]=0, \quad\left[\tau_{1}, \hat{g}(\mathcal{T}(T, \sigma), \pi, \sigma)\right]=0
$$

which allows us to rewrite the boundary conditions in Eq. (3.25) in terms of the reduced components $\hat{g}_{(u)}, \bar{u}=0,1$ of the group orbifold elements:

$$
\begin{aligned}
& {\left[\hat{g}_{(0)}(\mathcal{T}), \hat{g}_{(1)}(\mathcal{T})\right]=0, \quad \hat{g}_{(0)}(\mathcal{T})^{2}-\hat{g}_{(1)}(\mathcal{T})^{2}=\mathbb{1} \text { at } \xi=0}
\end{aligned}
$$

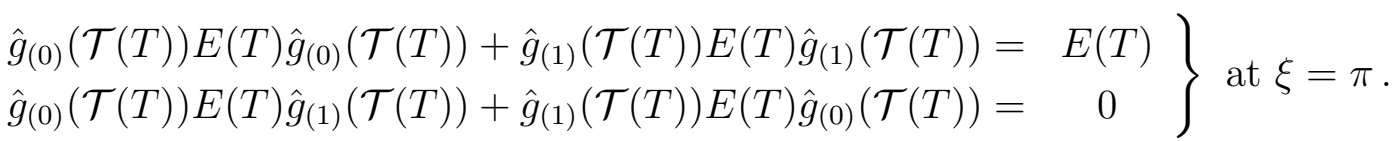

To see Eq. (3.28), begin by multiplying both sides of (3.25a and (3.25b) by $\hat{g}$; this set of boundary conditions can also be understood as the residuals at the boundaries of the non-local quadratic relations in Eq. (2.24). As above, the relations in (3.28b) apply only to the case $h_{\sigma}^{2}=1$. 
For the twisted tangent-space coordinates $\hat{\beta}$, we find the corresponding boundary conditions:

$$
\begin{gathered}
\hat{\beta}^{n(r) \mu 0}(0, \sigma)=0 \\
h_{\sigma}^{2}=1: \quad \hat{\beta}^{n(r) \mu u}(\pi, \sigma)=0 \text { unless } \frac{n(r)}{\rho(\sigma)} \in \mathbb{Z}+\frac{1}{2} .
\end{gathered}
$$

The result in Eq. (3.29a) holds for all $\hat{h}_{\sigma}$ because it is obtained directly from the world-sheet parity (2.22b) at $\xi=0$, while the following steps

$$
\hat{\beta}^{n(r) \mu u}(\pi, \sigma)=\hat{\beta}^{n(r) \mu u}(-\pi, \sigma) e^{2 \pi i\left(\frac{n(r)}{\rho(\sigma)}+\frac{u}{2}\right)}=-\hat{\beta}^{n(r) \mu u}(\pi, \sigma) e^{2 \pi i \frac{n(r)}{\rho(\sigma)}}
$$

give the result in Eq. (3.29b) for $h_{\sigma}^{2}=1$. We remark that the result in Eq. (3.29b) can be solved simply as $\hat{\beta}^{0 \mu u}(\pi, \sigma)=0$, but the more abstract language of (3.29b) will be useful below. With the tangent-space form of $\hat{g}$ in (2.22a $)$ and the $\mathcal{T}$-selection rule (2.14d), it is not difficult to check that the $\hat{\beta}$ boundary conditions (3.29) imply those of $\hat{g}(\mathcal{T})$ in Eq. (3.25).

The special orbits also allow us to derive boundary conditions for derivatives of the twisted fields, for example:

$$
\begin{gathered}
\left(\partial_{t} \hat{\beta}^{n(r) \mu 0}\right)(0, \sigma)=0 \\
h_{\sigma}^{2}=1: \quad\left(\partial_{t} \hat{\beta}^{n(r) \mu u}\right)(\pi, \sigma)=0 \text { unless } \frac{n(r)}{\rho(\sigma)} \in \mathbb{Z}+\frac{1}{2} \\
\left(\partial_{\xi} \hat{\beta}^{n(r) \mu 1}\right)(0, \sigma)=0 \\
h_{\sigma}^{2}=1: \quad\left(\partial_{\xi} \hat{\beta}^{n(r) \mu u}\right)(\pi, \sigma)=0 \text { unless } \frac{n(r)}{\rho(\sigma)} \in \mathbb{Z} .
\end{gathered}
$$

We note in particular that the $\partial_{t} \hat{\beta}$ boundary conditions are the same as those of $\hat{\beta}$. The boundary conditions obtained in this way for $\partial_{t} \hat{g}$ and $\partial_{\xi} \hat{g}$ are more complicated however, except when taken in the combinations which correspond to the twisted currents (see Subsec. 3.4) and the variational boundary conditions (see Subsec. 3.3).

We may also use these $h_{\sigma}^{2}=1$ monodromies to explicitly verify, for this specific case, various properties given above for all $\hat{h}_{\sigma}$. For example, the monodromies of the twisted currents

$$
\begin{aligned}
\hat{J}(\mathcal{T}(T, \sigma), \xi+2 \pi, t) & =\tau_{3} E(T, \sigma) \hat{J}(\mathcal{T}(T, \sigma), \xi, t) E^{*}(T, \sigma) \tau_{3} \\
\hat{\bar{J}}(\mathcal{T}(T, \sigma), \xi+2 \pi, t) & =\tau_{3} E(T, \sigma) \hat{\bar{J}}(\mathcal{T}(T, \sigma), \xi, t) E^{*}(T, \sigma) \tau_{3}
\end{aligned}
$$

follow from the $\hat{g}$ monodromies (2.25) and the $\hat{g}$ forms of the twisted currents in Eq. (3.15). The monodromies (3.32) agree with the general monodromies (3.22) of the twisted currents when $h_{\sigma}^{2}=1, E(T, \sigma)^{2}=1$. 
Finally, we explicitly check the variational boundary conditions (3.12b) and (3.13d) at $\xi=\pi$ :

$$
\hat{K}(\pi)=\hat{R}^{\xi}(\pi)=0
$$

In fact, these boundary conditions follow immediately when $h_{\sigma}^{2}=1$ from the world-sheet parities in Eq. (3.11) and the trivial monodromies

$$
\hat{K}(\xi+2 \pi)=\hat{K}(\xi), \quad \hat{R}^{A}(\xi+2 \pi)=\hat{R}^{A}(\xi)
$$

which themselves are obtained from the $\hat{g}$ monodromy. ${ }^{\ddagger 4}$

In what follows we will find, as illustrated above, that all boundary conditions at $\xi=0$ follow from world-sheet parity alone, and hence are true for all $\hat{h}_{\sigma}$.

\section{Einstein Geometry on the Solid Half Cylinder}

Our task in this section is to study the Einstein geometry, including the twisted Einstein tensors [24, of the open-string sectors of the WZW orientation orbifolds above.

\subsection{Two-Component WZW Geometry}

We begin this discussion in the untwisted theory, with the familiar ${ }^{\ddagger 5}$ geometric quantities of ordinary WZW models on the solid cylinder $\Gamma$ :

$$
\begin{gathered}
g(T, x(\xi))=e^{i x^{i}(\xi) e_{i}{ }^{a}(0) T_{a}}, \quad e_{i}{ }^{a}(0)=\delta_{i}{ }^{a}, \quad \partial_{i}=\frac{\partial}{\partial x^{i}} \\
e_{i}(T, x(\xi))=-i g^{-1}(T, x(\xi)) \partial_{i} g(T, x(\xi))=e(x(\xi))_{i}^{a} T_{a} \\
\bar{e}_{i}(T, x(\xi))=-i g(T, x(\xi)) \partial_{i} g^{-1}(T, x(\xi))=\bar{e}(x(\xi))_{i}{ }^{a} T_{a} \\
\bar{e}(x(\xi))_{i}{ }^{a}=-e(x(\xi))_{i}{ }^{b} \Omega(x(\xi))_{b}{ }^{a}, \quad g(T, x(\xi)) T_{a} g^{-1}(T, x(\xi))=\Omega(x(\xi))_{a}{ }^{b} T_{b} \\
\Omega(x(\xi))=g^{-1}\left(T^{a d j}, x(\xi)\right) \\
G_{i j}(x(\xi))=e(x(\xi))_{i}{ }^{a} G_{a b} e(x(\xi))_{j}{ }^{b}=\bar{e}(x(\xi))_{i}{ }^{a} G_{a b} \bar{e}(x(\xi))_{j}{ }^{b}, \quad \operatorname{Tr}\left(M T_{a} T_{b}\right)=G_{a b} \\
H_{i j k}(x(\xi))=\partial_{i} B_{j k}(x(\xi))+\operatorname{cyclic}=-i T r\left(M e_{i}(T, x(\xi))\left[e_{j}(T, x(\xi)), e_{k}(T, x(\xi))\right]\right) \\
=i \operatorname{Tr}\left(M \bar{e}_{i}(T, x(\xi))\left[\bar{e}_{j}(T, x(\xi)), \bar{e}_{k}(T, x(\xi))\right]\right)=e_{i}{ }^{a} e_{j}{ }^{b} e_{k}{ }^{c} f_{a b}{ }^{d} G_{d c}
\end{gathered}
$$

\footnotetext{
${ }^{\ddagger 4}$ Similarly, one easily checks from the $\hat{g}$ monodromies and Eq. (2.14g) that both integrands of the WZW orientation-orbifold action (3.9) are $2 \pi$-periodic; this conclusion holds of course only for $h_{\sigma}^{2}=1$.

${ }^{\ddagger}$ See for example Refs. 31, 32, 24].
} 


$$
\begin{gathered}
\gamma(\xi)=\epsilon^{A B C} \operatorname{Tr}\left(M g^{-1} \partial_{A} g g^{-1} \partial_{B} g g^{-1} \partial_{C} g\right)=\partial_{A} j^{A}(\xi) \\
j^{A}(\xi) \equiv \frac{3}{2} \epsilon^{A B C} \partial_{B} x^{i}(\xi) \partial_{C} x^{j}(\xi) B_{i j}(x(\xi)) \\
A=\{t, \xi, \rho\}, \quad \epsilon^{t \xi \rho}=1
\end{gathered}
$$

Here $e, \bar{e}, \Omega, G$ and $H$ are respectively the left- and right-invariant vielbeins, the adjoint action, the Einstein metric and the torsion field. Note that we have chosen the Einstein coordinates

$$
\left\{x^{i}=\beta^{a} e(0)_{a}^{i}, i=1 \ldots \operatorname{dim} g\right\}
$$

which correspond to $e(0)=\mathbb{1}$ at the origin. The identities in Eqs. (4.1h), (4.1i) are the standard Gauss' law which relates the winding-number current $j^{a}(\xi)$ to the density $\gamma(\xi)$ defined earlier in Eq. (3.5b).

Following Ref. 25] and our development above, we next define the corresponding twocomponent fields:

$$
\begin{aligned}
& x^{i \dot{I}}, \dot{I}=0,1: \quad x^{i 0}(\xi) \equiv x^{i}(\xi), \quad x^{i 1}(\xi) \equiv-x^{i}(-\xi) \\
& \partial_{i \dot{I}}(\xi) \equiv \frac{\partial}{\partial x^{i \dot{I}}(\xi)}, \quad \partial_{i \dot{I}}(\xi) x^{j \dot{J}}(\xi)=\delta_{i}^{j} \delta_{\dot{I}}^{j} \\
& \tilde{g}(T, x)=\left(\begin{array}{cc}
g\left(T, x^{0}(\xi)\right) & 0 \\
0 & g\left(T, x^{1}(\xi)\right)
\end{array}\right), \quad \partial_{ \pm} \tilde{g}(T, x)=\partial_{i \dot{I}} \tilde{g}(T, x) \partial_{ \pm} x^{i \dot{I}}(\xi) \\
& e_{i \dot{I}}(T, x) \equiv-i \tilde{g}^{-1}(T, x) \partial_{i \dot{I}} \tilde{g}(T, x) \equiv e(x)_{i \dot{I}}^{a \dot{J}} T_{a} \rho_{j} \\
& e(x)_{i \dot{I}}^{a \dot{J}}=-i \widehat{T r}\left(\left(M \otimes \mathbb{1}_{2}\right) \tilde{g}^{-1} \partial_{i \dot{I}} \tilde{g} G^{a b} \delta^{j \dot{K}} T_{b} \rho_{\dot{K}}\right)=\delta_{\dot{I}}^{\dot{J}} e\left(x^{\dot{I}}\right)_{i}{ }^{a} \\
& \tilde{g}(T, x) T_{a} \rho_{\dot{I}} \tilde{g}^{-1}(T, x)=\tilde{\Omega}(x)_{a \dot{I}}{ }^{b j} T_{b} \rho_{j}, \quad \tilde{\Omega}(x)=\tilde{g}^{-1}\left(T^{a d j}, x\right) \\
& \tilde{\Omega}(x)_{a \dot{I}}^{b \dot{J}}=\delta_{\dot{I}}^{\dot{J}} \Omega\left(x^{\dot{I}}\right)_{a}{ }^{b} \\
& G_{i \dot{I} ; j \dot{j}}(x) \equiv-\widehat{\operatorname{Tr}}\left(\left(M \otimes \mathbb{1}_{2}\right) \tilde{g}^{-1}(T, x) \partial_{i \dot{I}} \tilde{g}(T, x) \tilde{g}^{-1}(T, x) \partial_{j j} \tilde{g}(T, x(\xi))\right) \\
& =e(x)_{i \dot{I}}^{a \dot{K}} e(x)_{j \dot{J}}^{b \dot{L}} G_{a \dot{K}, b \dot{L}}, \quad G_{a \dot{K} ; b \dot{L}}=\delta_{\dot{K} \dot{L}} G_{a b} \\
& =\delta_{\dot{I} j} G_{i j}\left(x^{\dot{I}}\right)
\end{aligned}
$$




$$
\begin{aligned}
& B_{i \dot{I} ; j \dot{J}}(x)=\delta_{\dot{I} j} B_{i j}\left(x^{\dot{I}}\right) \\
& H_{i \dot{I} ; j \dot{j} ; k \dot{K}}(x) \equiv \partial_{i \dot{I}} B_{j \dot{j} ; k \dot{K}}(x)+\text { cyclic } \\
& =-i \widehat{\operatorname{Tr}}\left(\left(M \otimes \mathbb{1}_{2}\right) e_{i \dot{I}}(T, x)\left[e_{j \dot{j}}(T, x), e_{k \dot{K}}(T, x)\right]\right) \\
& =e_{i \dot{I}}^{a \dot{L}} e_{j \dot{J}}^{b \dot{M}} e_{k \dot{K}}{ }^{c \dot{N}} f_{a \dot{L} ; b \dot{M} ; c \dot{N}}, \quad f_{a \dot{I} ; b \dot{j} ; c \dot{K}}=\delta_{\dot{I} \dot{j}} \delta_{\dot{J} \dot{K}} f_{a b}{ }^{d} G_{d c} \\
& =e\left(x^{\dot{I}}\right)_{i}{ }^{a} e\left(x^{\dot{I}}\right)_{j}{ }^{b} e\left(x^{\dot{I}}\right)_{k}{ }^{c} f_{a b}{ }^{d} G_{d c}=\delta_{\dot{I} j} \delta_{j \dot{K} \dot{K}} H_{i j k}\left(x^{\dot{I}}\right) \\
& \epsilon^{A B C} \widehat{\operatorname{Tr}}\left(\left(M \otimes \mathbb{1}_{2}\right) \tilde{g}^{-1}(T, x) \partial_{A} \tilde{g}(T, x) \tilde{g}^{-1}(T, x) \partial_{B} \tilde{g}(T, x) \tilde{g}^{-1}(T, x) \partial_{C} \tilde{g}(T, x)\right) \\
& =\partial_{A} \tilde{j}^{A}(\xi) \\
& \tilde{j}^{A}(\xi) \equiv \frac{3}{2} \epsilon^{A B C} \partial_{B} x^{i \dot{I}}(\xi) \partial_{C} x^{j \dot{J}}(\xi) B_{i \dot{I} ; j \dot{j}}(x(\xi)) \text {. }
\end{aligned}
$$

The matrices $\rho_{\dot{I}}$ are defined in Eq. (2.9b). We emphasize that, although many of the fields carry more than one two-component index $\dot{I}, \dot{J}$, each field is diagonal in these indices and therefore has only two independent components in the two-dimensional space.

From the definitions of these two-component fields, one finds the following behavior under world-sheet parity $\xi \rightarrow-\xi$ :

$$
\begin{aligned}
& x^{i \dot{I}}(-\xi)=-x^{i \dot{J}}(\xi)\left(\tau_{1}\right)_{\dot{J}}^{\dot{I}}, \quad \partial_{i \dot{I}}(-\xi)=-\left(\tau_{1}\right)_{\dot{I}}^{\dot{j}} \partial_{i \dot{j}}(\xi) \\
& \tilde{\Omega}(x(-\xi))_{a \dot{I}}^{b \dot{J}}=\left(\tau_{1}\right)_{\dot{I}}^{\dot{K}} \tilde{\Omega}^{-1}(x(\xi))_{a \dot{K}}{ }^{b \dot{L}}\left(\tau_{1}\right)_{\dot{L}}{ }^{j} \\
& e(x(-\xi))_{i \dot{I}}^{a \dot{J}}=\left(\tau_{1}\right)_{\dot{I}}^{\dot{K}}(e(x(\xi)) \tilde{\Omega}(x(\xi)))_{i \dot{K}}^{a \dot{L}}\left(\tau_{1}\right)_{\dot{L}}^{\dot{J}} \\
& G_{i \dot{I} ; j \dot{J}}(x(-\xi))=\left(\tau_{1}\right)_{\dot{I}}^{\dot{K}}\left(\tau_{1}\right)_{\dot{J}}^{\dot{L}} G_{i \dot{K} ; j \dot{L}}(x(\xi)) \\
& H_{i \dot{I} ; j \dot{j} ; k \dot{K}}(x(-\xi))=\left(\tau_{1}\right)_{\dot{I}}{ }^{\dot{L}}\left(\tau_{1}\right)_{\dot{J}}^{\dot{M}}\left(\tau_{1}\right)_{\dot{K}}{ }^{\dot{N}} H_{i \dot{L} ; j \dot{M} ; k \dot{N}}(x(\xi)) \\
& B_{i \dot{I} ; j \dot{J}}(x(-\xi))=-\left(\tau_{1}\right)_{\dot{I}}^{\dot{K}}\left(\tau_{1}\right)_{\dot{J}}^{\dot{L}} B_{i \dot{K} ; j \dot{L}}(x(\xi)) \\
& \tilde{j}^{\xi}(-\xi)=-\tilde{j}^{\xi}(\xi), \quad \tilde{j}^{t, \rho}(-\xi)=\tilde{j}^{t, \rho}(\xi) .
\end{aligned}
$$

In particular, the results in Eqs. (4.4c-g) follow by using the world-sheet parity (2.9d) in the relevant trace formulae in Eq. (4.3).

Taken together, the diagonal forms in Eq. (4.3) and the world-sheet parities (4.4) imply consistency relations such as

$$
G_{i j}\left(x^{\dot{I}}(-\xi)\right)=\left(\tau_{1}\right)_{\dot{I}}^{\dot{j}} G_{i j}\left(x^{\dot{j}}(\xi)\right), \quad B_{i j}\left(x^{\dot{I}}(-\xi)\right)=-\left(\tau_{1}\right)_{\dot{I}}^{\dot{j}} B_{i j}\left(x^{\dot{J}}(\xi)\right)
$$


which record in our notation (see Eq. (4.3a)) the following behavior of the original fields under (target-space) space-time parity $x \leftrightarrow-x$ :

$$
\begin{gathered}
g(T,-x)=g^{-1}(T, x), \quad \Omega(-x)=\Omega^{-1}(x), \quad e(-x)_{i}{ }^{a}=-\bar{e}(x)_{i}{ }^{a} \\
G_{i j}(-x)=G_{i j}(x), \quad B_{i j}(-x)=-B_{i j}(x), \quad H_{i j k}(-x)=H_{i j k}(x) .
\end{gathered}
$$

These space-time parities hold in the general WZW model, as is easily checked from the following explicit forms [32, 24] of the geometric quantities:

$$
\begin{gathered}
\Omega(x)=e^{-i Y(x)} \\
e(x)_{i}{ }^{a}=e(0)_{i}^{b}\left(\frac{e^{i Y(x)}-1}{i Y(x)}\right)_{b}^{a}, \quad \bar{e}(x)_{i}{ }^{a}=e(0)_{i}{ }^{b}\left(\frac{e^{-i Y(x)}-1}{i Y(x)}\right)_{b}^{a} \\
G_{i j}(x)=e(0)_{i}{ }^{a}\left(\frac{e^{i Y(x)}+e^{-i Y(x)}-2}{(i Y(x))^{2}}\right)_{a}^{b} G_{b c} e(0)_{j}{ }^{c} \\
B_{i j}(x)=e(0)_{i}{ }^{a}\left(\frac{e^{i Y(x)}-e^{-i Y(x)}-2 i Y(x)}{(i Y(x))^{2}}\right)_{a}^{b} G_{b c} e(0)_{j}{ }^{c} \\
Y(x) \equiv x^{i} e(0)_{i}{ }^{a} T_{a}^{a d j}, \quad\left(T_{a}^{a d j}\right)_{b}{ }^{c}=-i f_{a b}{ }^{c} .
\end{gathered}
$$

Although the space-time parities (4.6) appear here as a byproduct of our formulation, we will find below and in Sec. 5 that space-time parity in fact plays a fundamental role in the construction of orientation orbifolds.

\subsection{From the Solid Half Cylinder to the Strip}

Using the two component fields of the previous subsection, we turn next to find the twodimensional or sigma-model form of the half-cylinder action in Eq. (3.4).

For this discussion, we note first the boundary conditions on the two-component windingnumber current $\tilde{j}(\xi)$ in Eq. (4.31)

$$
\tilde{j}^{\xi}(0)=\tilde{j}^{\xi}(\pi)=0
$$

which are obtained from $2 \pi$-periodicity of the untwisted fields and the world-sheet parity (4.4g) of $\tilde{j}(\xi)$. It follows that

$$
\int_{y=0} d t d x\left(-\vec{e}_{y} \cdot \tilde{j}\right)=\int_{y=0} d t d x\left(\tilde{j}^{\xi}(\pi)-\tilde{j}^{\xi}(0)\right)=0
$$

so, with the Gauss' law (4.3k), one sees for the WZW term that the contribution from the flat side of the solid half cylinder vanishes. Then one finds the two-dimensional form of the 
WZW term on $\Gamma_{1 / 2}$ :

$$
\int_{\Gamma_{\frac{1}{2}}} \widehat{\operatorname{Tr}}\left(\left(M \otimes \mathbb{1}_{2}\right)\left(\tilde{g}^{-1}(T, \xi) d \tilde{g}(T, \xi)\right)^{3}\right)=-\frac{3}{2} \int d t \int_{0}^{\pi} d \xi B_{i \dot{i} ; j \dot{j}}(x) \partial_{+} x^{i \dot{I}} \partial_{-} x^{j \dot{j}} .
$$

With this result and Eq. (4.3h) for the kinetic term, we finally obtain the sigma-model form of the WZW action on the strip

$$
\begin{aligned}
S_{W Z W}^{\text {strip }} & =\frac{1}{8 \pi} \int d t \int_{0}^{\pi} d \xi\left(G_{i \dot{I} ; j \dot{j}}(x)+B_{i \dot{I} ; j \dot{j}}(x)\right) \partial_{+} x^{i \dot{I}} \partial_{-} x^{j \dot{j}} \\
& =\frac{1}{8 \pi} \int d t \int_{0}^{\pi} d \xi \sum_{\dot{I}=0}^{1}\left(G_{i j}\left(x^{\dot{I}}\right)+B_{i j}\left(x^{\dot{I}}\right)\right) \partial_{+} x^{i \dot{I}} \partial_{-} x^{j \dot{J}} \\
& =S_{W Z W}\left[M \otimes \mathbb{1}_{2}, \tilde{g} ; \Gamma_{\frac{1}{2}}\right]
\end{aligned}
$$

where $G_{i j}(x)$ and $B_{i j}(x)$ are the original WZW fields.

It is instructive to check that the two-component action (4.11) on the strip is equivalent to the standard sigma-model form of the WZW action on the cylinder:

$$
S_{W Z W}=\frac{1}{8 \pi} \int d t \int_{0}^{2 \pi} d \xi\left(G_{i j}(x(\xi))+B_{i j}(x(\xi))\right) \partial_{+} x^{i}(\xi) \partial_{-} x^{j}(\xi) .
$$

To see this equivalence, begin with the form of the strip action in Eq. (4.11b) and use the definition of $x^{\dot{I}}$ in Eq. (4.3a) to follow the steps

$$
\begin{gathered}
S_{W Z W}^{\text {strip }}=\frac{1}{8 \pi} \int d t \int_{0}^{\pi} d \xi\left[\left(G_{i j}(x(\xi))+B_{i j}(x(\xi))\right) \partial_{+} x^{i}(\xi) \partial_{-} x^{j}(\xi)\right. \\
\left.+\left(G_{i j}(-x(-\xi))+B_{i j}(-x(-\xi))\right) \partial_{+} x^{i}(-\xi) \partial_{-} x^{j}(-\xi)\right] \\
=\frac{1}{8 \pi} \int d t \int_{0}^{\pi} d \xi\left[\left(G_{i j}(x(\xi))+B_{i j}(x(\xi))\right) \partial_{+} x^{i}(\xi) \partial_{-} x^{j}(\xi)\right. \\
\left.+\left(G_{i j}(x(-\xi))-B_{i j}(x(-\xi))\right) \partial_{+} x^{i}(-\xi) \partial_{-} x^{j}(-\xi)\right] \\
=\frac{1}{8 \pi} \int d t \int_{0}^{\pi} d \xi\left[\left(G_{i j}(x(\xi))+B_{i j}(x(\xi))\right) \partial_{+} x^{i}(\xi) \partial_{-} x^{j}(\xi)\right. \\
\left.+\left(G_{i j}(x(-\xi))+B_{i j}(x(-\xi))\right) \partial_{-} x^{i}(-\xi) \partial_{+} x^{j}(-\xi)\right] \\
=\frac{1}{8 \pi} \int d t\left(\int_{0}^{\pi}+\int_{-\pi}^{0}\right) d \xi\left(G_{i j}(x(\xi))+B_{i j}(x(\xi))\right) \partial_{+} x^{i}(\xi) \partial_{-} x^{j}(\xi)=S_{W Z W}
\end{gathered}
$$

where (4.13b) is obtained from (4.13a) by the space-time parities (4.6b). The last form in Eq. (4.13d) is indeed equal to the standard form (4.12) of the sigma model action on the cylinder - because the integrands of both are $2 \pi$-periodic. 


\subsection{Automorphic Responses of the Geometric Quantities}

We turn next to the action of the general orientation-reversing automorphism $\hat{h}_{\sigma}$ on the two-component geometric quantities:

$$
\begin{aligned}
& \omega\left(h_{\sigma}\right)_{i}{ }^{j} \equiv e(0)_{i}{ }^{a} \omega\left(h_{\sigma}\right)_{a}{ }^{b} e(0)_{b}{ }^{j}, \quad \omega\left(h_{\sigma}\right) \in \operatorname{Aut}(g) \\
& x^{i \dot{I}}(\xi)^{\prime}=x^{j \dot{J}}(\xi)\left(\tau_{1}\right)_{j}{ }_{j}^{\dot{I}} \omega^{\dagger}\left(h_{\sigma}\right)_{j}{ }^{i}, \quad \partial_{i \dot{I}}(\xi)^{\prime}=\omega\left(h_{\sigma}\right)_{i}{ }^{j}\left(\tau_{1}\right)_{\dot{I}}{ }^{j} \partial_{j \dot{J}}, \quad \dot{I}=0,1 \\
& \tilde{\Omega}(x)_{a \dot{I}}{ }^{b j \prime}=\tilde{\Omega}\left(x^{\prime}\right)_{a \dot{I}}{ }^{b j}=\omega\left(h_{\sigma}\right)_{a}{ }^{c}\left(\tau_{1}\right)_{\dot{I}}{ }^{\dot{K}} \tilde{\Omega}(x)_{c \dot{K}}{ }^{d \dot{L}}\left(\tau_{1}\right)_{\dot{L}}{ }^{j} \omega^{\dagger}\left(h_{\sigma}\right)_{d}{ }^{b} \\
& e_{i \dot{I}}(T, x)^{\prime}=e_{i \dot{I}}\left(T, x^{\prime}\right)=\omega\left(h_{\sigma}\right)_{i}^{j}\left(\tau_{1}\right)_{\dot{I}}{ }^{j} \tau_{1} W\left(h_{\sigma} ; T\right) e_{j j}(T, x) W^{\dagger}\left(h_{\sigma} ; T\right) \tau_{1} \\
& e(x)_{i \dot{I}}^{a j \prime}=e\left(x^{\prime}\right)_{i \dot{I}}^{a j}=\omega\left(h_{\sigma}\right)_{i}{ }^{j}\left(\tau_{1}\right)_{\dot{I}}^{\dot{K}} e(x)_{j \dot{K}}{ }^{b \dot{L}}\left(\tau_{1}\right)_{\dot{L}}{ }^{j} \omega^{\dagger}\left(h_{\sigma}\right)_{b}{ }^{a} \\
& G_{i \dot{i} ; j \dot{j}}(x)^{\prime}=G_{i \dot{i} ; j \dot{j}}\left(x^{\prime}\right)=\omega\left(h_{\sigma}\right)_{i}{ }^{k} \omega\left(h_{\sigma}\right)_{j}{ }^{l}\left(\tau_{1}\right)_{\dot{I}}{ }^{\dot{K}}\left(\tau_{1}\right)_{\dot{J}}{ }^{\dot{L}} G_{k \dot{K} ; l \dot{L}}(x) \\
& B_{i \dot{i} ; j \dot{j}}(x)^{\prime}=B_{i \dot{i} ; j \dot{j}}\left(x^{\prime}\right)=\omega\left(h_{\sigma}\right)_{i}{ }^{k} \omega\left(h_{\sigma}\right)_{j}{ }^{l}\left(\tau_{1}\right)_{\dot{I}}{ }^{\dot{K}}\left(\tau_{1}\right)_{j}{ }^{\dot{ }} B_{k \dot{K} ; \dot{L}}(x) \\
& H_{i \dot{i} ; j j ; j \dot{K}}(x)^{\prime}=H_{i \dot{I} ; j j ; j \dot{K}}\left(x^{\prime}\right) \\
& =\omega\left(h_{\sigma}\right)_{i}{ }^{l} \omega\left(h_{\sigma}\right)_{j}{ }^{m} \omega\left(h_{\sigma}\right)_{k}{ }^{n}\left(\tau_{1}\right)_{\dot{I}}^{\dot{L}}\left(\tau_{1}\right)_{\dot{J}}^{\dot{M}}\left(\tau_{1}\right)_{\dot{K}}{ }^{\dot{N}} H_{l \dot{L} ; m \dot{M} ; n \dot{N}}(x) .
\end{aligned}
$$

These automorphic responses follow by substitution of Eqs. (2.9g-i) into (4.2) and the $\widehat{T r}$ formulae in Eq. (4.3). As expected, the strip form (4.11a) of the WZW sigma-model action is invariant under the general orientation-reversing automorphism above.

Finally, it is instructive to consider the action of $\hat{h}_{\sigma}$ on the original fields:

$$
\begin{gathered}
x^{i}(\xi)^{\prime}=-x^{j}(-\xi) \omega^{\dagger}\left(h_{\sigma}\right)_{j}{ }^{i} \\
g(T, x(\xi))^{\prime}=g\left(T, x^{\prime}(\xi)\right)=g\left(T,-x(-\xi) \omega^{\dagger}\right)=W\left(h_{\sigma} ; T\right) g(T,-x(-\xi)) W^{\dagger}\left(h_{\sigma} ; T\right) \\
\Omega(x(\xi))_{a}{ }^{b \prime}=\Omega\left(-x(-\xi) \omega^{\dagger}\right)_{a}{ }^{b}=\omega\left(h_{\sigma}\right)_{a}{ }^{c} \Omega(-x(-\xi))_{c}{ }^{d} \omega^{\dagger}\left(h_{\sigma}\right)_{d}{ }^{b} \\
e(x(\xi))_{i}{ }^{\prime \prime}=e\left(-x(-\xi) \omega^{\dagger}\right)_{i}{ }^{a}=\omega\left(h_{\sigma}\right)_{i}{ }^{j} e(-x(-\xi))_{j}{ }^{b} \omega^{\dagger}\left(h_{\sigma}\right)_{b}{ }^{a} \\
G_{i j}(x(\xi))^{\prime}=G_{i j}\left(-x(-\xi) \omega^{\dagger}\right)=\omega\left(h_{\sigma}\right)_{i}{ }^{k} \omega\left(h_{\sigma}\right)_{j}{ }^{l} G_{k l}(-x(-\xi)) \\
B_{i j}(x(\xi))^{\prime}=B_{i j}\left(-x(-\xi) \omega^{\dagger}\right)=\omega\left(h_{\sigma}\right)_{i}{ }^{k} \omega\left(h_{\sigma}\right)_{j}{ }^{l} B_{k l}(-x(-\xi)) \\
H_{i j k}(x(\xi))^{\prime}=H_{i j k}\left(-x(-\xi) \omega^{\dagger}\right)=\omega\left(h_{\sigma}\right)_{i}{ }^{l} \omega\left(h_{\sigma}\right)_{j}{ }^{m} \omega\left(h_{\sigma}\right)_{k}{ }^{n} H_{l m n}(-x(-\xi)) .
\end{gathered}
$$

We note in particular another set of consistency relations on the original fields

$$
\begin{gathered}
g\left(T, x \omega^{\dagger}\right)=W(T) g(T, x) W^{\dagger}(T) \\
\Omega\left(x \omega^{\dagger}\right)_{a}{ }^{b}=\omega_{a}{ }^{c} \Omega(x)_{c}{ }^{d}\left(\omega^{\dagger}\right)_{d}{ }^{b}, \quad e\left(x \omega^{\dagger}\right)_{i}{ }^{a}=\omega_{i}{ }^{j} e(x)_{j}{ }^{b}\left(\omega^{\dagger}\right)_{b}{ }^{a}
\end{gathered}
$$




$$
\begin{array}{r}
G_{i j}\left(x \omega^{\dagger}\right)=\omega_{i}^{k} \omega_{j}^{l} G_{k l}(x), \quad B_{i j}\left(x \omega^{\dagger}\right)=\omega_{i}^{k} \omega_{j}^{l} B_{k l}(x) \\
H_{i j k}\left(x \omega^{\dagger}\right)=\omega_{i}^{l} \omega_{j}^{m} \omega_{k}^{n} H_{l m n}(x) \\
\omega \equiv \omega\left(h_{\sigma}\right), \quad W(T) \equiv W\left(h_{\sigma} ; T\right), \quad h_{\sigma} \in \operatorname{Aut}(g)
\end{array}
$$

which are implied by comparing the last two entries of each line in Eq. (4.15). This set of consistency relations expresses the Lie symmetry of the WZW models, and these relations can be easily checked from the explicit forms given in Eq. (4.7). In fact, as we will emphasize in Subsec. 5.1, such consistency relations together with the behavior of the fields under space-time parity can be considered as the fundamental symmetries which allow orientation-reversing automorphisms.

\subsection{Geometric Eigenfields}

Following the familiar procedure [3, 5, 14, 24, 25] of the orbifold program, we now define the eigenfields associated to the two-component Einstein coordinates and tensors

$$
\begin{aligned}
& U(\sigma)_{n(r) \mu}{ }^{i}=U(\sigma)_{n(r) \mu}{ }^{a} e(0)_{a}{ }^{i}, \quad U^{\dagger}(\sigma)_{i}{ }^{n(r) \mu}=e(0)_{i}{ }^{a} U^{\dagger}(\sigma)_{a}{ }^{n(r) \mu} \\
& X_{\sigma}^{n(r) \mu u}(\xi) \equiv x^{i \dot{I}}(\xi) \chi(\sigma)_{n(r) \mu}^{-1} U^{\dagger}(\sigma)_{i}^{n(r) \mu}\left(\frac{1}{\sqrt{2}} U^{\dagger}{ }^{u}\right), \quad \bar{u}=0,1 \\
& \partial_{n(r) \mu u}(\xi) \equiv \frac{\partial}{\partial X^{n(r) \mu u}(\xi)}=\chi(\sigma)_{n(r) \mu} U(\sigma)_{n(r) \mu}{ }^{i}\left(\sqrt{2} U_{u}{ }^{\dot{I}}\right) \partial_{i \dot{I}}(\xi) \\
& \partial_{n(r) \mu u}(\xi) X_{\sigma}^{n(s) \nu v}(\xi)=\delta_{n(r)-n(s), 0 \bmod \rho(\sigma)} \delta_{\mu}^{\nu} \delta_{u-v, 0 \bmod 2} \\
& \mathcal{W}(X)_{n(r) \mu u}{ }^{n(s) \nu v} \equiv \chi(\sigma)_{n(r) \mu} U(\sigma)_{n(r) \mu}{ }^{a}\left(\sqrt{2} U_{u}{ }^{\dot{I}}\right) \tilde{\Omega}(x(X))_{a \dot{I}}{ }^{b j} \\
& \times \chi(\sigma)_{n(s) \nu}^{-1} U^{\dagger}(\sigma)_{b}{ }^{n(s) \nu}\left(\frac{1}{\sqrt{2}}\left(U^{\dagger}\right)_{j}{ }^{v}\right) \\
& \mathcal{E}_{n(r) \mu u}(\mathcal{T}, \mathcal{X}) \equiv \chi(\sigma)_{n(r) \mu} U(\sigma)_{n(r) \mu}{ }^{i}\left(\sqrt{2} U_{u}{ }^{\dot{I}}\right) U(T, \sigma) U e_{i \dot{I}}(T, x(X)) U^{\dagger} U^{\dagger}(T, \sigma) \\
& \mathcal{E}(X)_{n(r) \mu u^{n(s) \nu v}} \equiv \chi(\sigma)_{n(r) \mu} U(\sigma)_{n(r) \mu}{ }^{i}\left(\sqrt{2} U_{u}{ }^{\dot{I}}\right) e(x(X))_{i \dot{I}}{ }^{a j} \\
& \times \chi(\sigma)_{n(s) \nu}^{-1} U^{\dagger}(\sigma)_{a}^{n(s) \nu}\left(\frac{1}{\sqrt{2}}\left(U^{\dagger}\right)_{j}{ }^{v}\right) \\
& \mathcal{G}_{n(r) \mu u ; n(s) \nu v}(X) \equiv \chi(\sigma)_{n(r) \mu} \chi(\sigma)_{n(s) \nu} U(\sigma)_{n(r) \mu}^{i} U(\sigma)_{n(s) \nu}{ }^{j}\left(\sqrt{2} U_{u}^{\dot{I}}\right)\left(\sqrt{2} U_{v}^{j}\right) G_{i \dot{i} ; j j}(x(X)) \\
& \mathcal{B}_{n(r) \mu u ; n(s) \nu v}(X) \equiv \chi(\sigma)_{n(r) \mu} \chi(\sigma)_{n(s) \nu} U(\sigma)_{n(r) \mu}{ }^{i} U(\sigma)_{n(s) \nu}{ }^{j}\left(\sqrt{2} U_{u}^{\dot{I}}\right)\left(\sqrt{2} U_{v}^{j}\right) B_{i \dot{I} ; j j}(x(X)) \\
& \mathcal{H}_{n(r) \mu u ; n(s) \nu v ; n(t) \delta w}(X) \equiv \chi(\sigma)_{n(r) \mu} \chi(\sigma)_{n(s) \nu} \chi(\sigma)_{n(t) \delta} U(\sigma)_{n(r) \mu}{ }^{i} U(\sigma)_{n(s) \nu}{ }^{j} U(\sigma)_{n(t) \delta}{ }^{k} \\
& \times\left(\sqrt{2} U_{u}^{\dot{I}}\right)\left(\sqrt{2} U_{v}{ }^{j}\right)\left(\sqrt{2} U_{w}{ }^{\dot{K}}\right) H_{i \dot{i} ; j \dot{j} ; k \dot{K}}(x(X))
\end{aligned}
$$


where the unitary matrices $U(\sigma), U(T, \sigma)$ and $U$ are defined in Eqs. (2.11), (2.16e) and $\chi(\sigma)_{n(r) \mu}$ are the same normalization constants which appear in Eq. (2.14).

Relations among these eigenfields include the following

$$
\begin{aligned}
& \mathcal{G}(\mathcal{T}, X) \mathcal{T}_{n(r) \mu u} \mathcal{G}^{-1}(\mathcal{T}, X)=\mathcal{W}(X)_{n(r) \mu u}{ }^{n(s) \nu v} \mathcal{T}_{n(s) \nu v} \\
& \mathcal{E}_{n(r) \mu u}(\mathcal{T}, X)=-i \mathcal{G}^{-1}(\mathcal{T}, X) \partial_{n(r) \mu u} \mathcal{G}(\mathcal{T}, X)=\mathcal{E}(X)_{n(r) \mu u}{ }^{n(s) \nu v} \mathcal{T}_{n(s) \nu v} \\
& \mathcal{G}_{n(r) \mu u ; n(s) \nu v}(\mathcal{X})=-\widehat{\operatorname{Tr}}\left(\left(\mathcal{M} \otimes \mathbb{1}_{2}\right)\left(\mathcal{G}^{-1} \partial_{n(r) \mu u} \mathcal{G G}^{-1} \partial_{n(s) \nu v} \mathcal{G}\right)\right) \\
& =\mathcal{E}(X)_{n(r) \mu u}{ }^{n\left(r^{\prime}\right) \mu^{\prime} u^{\prime}} \mathcal{E}(\mathcal{X})_{n(s) \nu v}{ }^{n\left(s^{\prime}\right) \nu^{\prime} v^{\prime}} \mathcal{G}_{n\left(r^{\prime}\right) \mu^{\prime} u^{\prime} ; n\left(s^{\prime}\right) \nu^{\prime} v^{\prime}}(\sigma) \\
& \mathcal{H}_{n(r) \mu u ; n(s) \nu v ; n(t) \delta w}(\mathcal{X})=\partial_{n(r) \mu u} \mathcal{B}_{n(s) \nu v ; n(t) \delta w}(\mathcal{X})+\text { cyclic }
\end{aligned}
$$

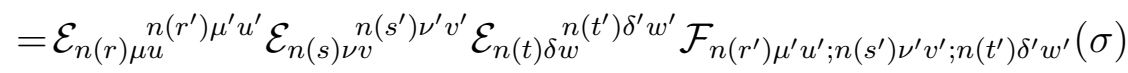

$$
\begin{aligned}
& \mathcal{F}_{n(r) \mu u ; n(s) \nu v ; n(t) \delta w}(\sigma)=2 \delta_{u+v+w, 0 \bmod 2} \mathcal{F}_{n(r) \mu ; n(s) \nu ; n(t) \delta}(\sigma) \\
& \mathcal{F}_{n(r) \mu ; n(s) \nu ; n(t) \delta}(\sigma)=\mathcal{F}_{n(r) \mu ; n(s) \nu}{ }^{n\left(t^{\prime}\right), \delta^{\prime}}(\sigma) \mathcal{G}_{n\left(t^{\prime}\right), \delta^{\prime} ; n(t) \delta}(\sigma) \\
& \epsilon^{A B C} \widehat{\operatorname{Tr}}\left((\mathcal{M} \otimes \mathbb{1})\left(\mathcal{G}^{-1} \partial_{A} \mathcal{G G}^{-1} \partial_{B} \mathcal{G} \mathcal{G}^{-1} \partial_{C} \mathcal{G}\right)\right)=\partial_{A} \tilde{j}^{A}(\xi) \\
& \tilde{j}^{A}(\xi)=\frac{3}{2} \epsilon^{A B C} \partial_{B} X^{n(r) \mu u}(\xi) \partial_{C} X^{n(s) \nu v}(\xi) \mathcal{B}_{n(r) \mu u ; n(s) \nu v}(X(\xi))
\end{aligned}
$$

where the eigengroup element, the total twisted metric and the ordinary twisted metric were defined in Eqs. (2.16a), (2.19b) and (2.14) respectively. The $\mathcal{F}(\sigma)$ on the right side of Eq. (4.18ed) is the totally antisymmetric form of the ordinary twisted structure constants defined in Eq. (2.14).

As above, these eigenfields are constructed to have diagonal responses to the orientationreversing automorphism:

$$
\begin{gathered}
X_{\sigma}^{n(r) \mu u}(\xi)^{\prime}=X_{\sigma}^{n(r) \mu u}(\xi) e^{2 \pi i\left(\frac{n(r)}{\rho(\sigma)}+\frac{u}{2}\right)}, \quad \partial_{n(r) \mu u}(\xi)^{\prime}=e^{-2 \pi i\left(\frac{n(r)}{\rho(\sigma)}+\frac{u}{2}\right)} \partial_{n(r) \mu u}(\xi) \\
\mathcal{E}_{n(r) \mu u}(\mathcal{T}, X)^{\prime}=\mathcal{E}_{n(r) \mu u}\left(\mathcal{T}, X^{\prime}\right)=e^{-2 \pi i\left(\frac{n(r)}{\rho(\sigma)}+\frac{u}{2}\right)} E(T, \sigma) \mathcal{E}_{n(r) \mu u}(\mathcal{T}, X) E^{*}(T, \sigma) \\
\mathcal{E}(X)_{n(r) \mu u}^{n(s) \nu v \prime}=\mathcal{E}\left(X^{\prime}\right)_{n(r) \mu u}^{n(s) \nu v}=e^{-2 \pi i\left(\frac{n(r)-n(s)}{\rho(\sigma)}+\frac{u-v}{2}\right)} \mathcal{E}(X)_{n(r) \mu u}{ }^{n(s) \nu v} \\
\mathcal{G}_{n(r) \mu u ; n(s) \nu v}(X)^{\prime}=\mathcal{G}_{n(r) \mu u ; n(s) \nu v}\left(X^{\prime}\right)=e^{-2 \pi i\left(\frac{n(r)+n(s)}{\rho(\sigma)}+\frac{u+v}{2}\right)} \mathcal{G}_{n(r) \mu u ; n(s) \nu v}(X) \\
\mathcal{B}_{n(r) \mu u ; n(s) \nu v}(X)^{\prime}=\mathcal{B}_{n(r) \mu u ; n(s) \nu v}\left(X^{\prime}\right)=e^{-2 \pi i\left(\frac{n(r)+n(s)}{\rho(\sigma)}+\frac{u+v}{2}\right)} \mathcal{B}_{n(r) \mu u ; n(s) \nu v}(X) \\
\mathcal{H}_{n(r) \mu u ; n(s) \nu v ; n(t) \delta w}(X)^{\prime}=\mathcal{H}_{n(r) \mu u ; n(s) \nu v ; n(t) \delta w}\left(X^{\prime}\right) \\
=e^{-2 \pi i\left(\frac{n(r)+n(s)+n(t)}{\rho(\sigma)}+\frac{u+v+w}{2}\right)} \mathcal{H}_{n(r) \mu u ; n(s) \nu v ; n(t) \delta w}(X)
\end{gathered}
$$


To obtain these responses, we have used the action (4.14) of $\hat{h}_{\sigma}$, the definitions in Eq. (4.17) and the $H$-eigenvalue problems in Eq. (2.11).

Using the explicit forms (4.7) of the original fields, these eigenfields can also be explicitly evaluated as

$$
\begin{gathered}
\mathcal{W}(X)=\mathcal{G}^{-1}\left(\tilde{\mathcal{T}}^{a d j}, X\right)=e^{-i \mathcal{Y}(X)}, \quad \mathcal{Y}(\mathcal{X}) \equiv X_{\sigma}^{n(r) \mu u} \tilde{\mathcal{T}}_{n(r) \mu}^{a d j} \tau_{u} \\
\left(\tilde{\mathcal{T}}_{n(r) \mu}^{a d j}\right)_{n(s) \nu}{ }^{n(t) \delta} \equiv-i \mathcal{F}_{n(r) \mu ; n(s) \nu}{ }^{n(t) \delta}(\sigma)=\chi(\sigma)_{n(s) \nu} \chi(\sigma)_{n(t) \delta}^{-1} \mathcal{T}_{n(r) \mu}\left(T^{a d j}, \sigma\right)_{n(s) \nu}{ }^{n(t) \delta} \\
{\left[\tilde{\mathcal{T}}_{n(r) \mu}^{a d j}, \tilde{\mathcal{T}}_{n(s) \nu}^{a d j}\right]=i \mathcal{F}_{n(r) \mu ; n(s) \nu}{ }^{n(r)+n(s), \delta}(\sigma) \tilde{\mathcal{T}}_{n(r)+n(s), \delta}^{a d j}} \\
\mathcal{E}(\mathcal{X})_{n(r) \mu u}^{n(s) \nu v}=\left(\frac{e^{i \mathcal{Y}(X)}-1}{i \mathcal{Y}(x)}\right)_{n(r) \mu u}^{n(s) \nu v}, \quad \mathcal{E}(0)=\mathbb{1} \\
\mathcal{G}_{n(r) \mu u ; n(s) \nu v}(\mathcal{X})=\left(\frac{e^{i \mathcal{Y}(x)+e^{-i \mathcal{Y}(x)}-2}}{(i \mathcal{Y}(x))^{2}}\right)_{n(r) \mu u}^{n(t) \delta w} \mathcal{G}_{n(t) \delta w ; n(s) \nu v}(\sigma) \\
\mathcal{B}_{n(r) \mu u ; n(s) \nu v}(\mathcal{X})=\left(\frac{e^{i \mathcal{Y}(x)}-e^{-i \mathcal{Y}(x)}-2 i \mathcal{Y}(x)}{(i \mathcal{Y}(x))^{2}}\right)_{n(r) \mu u}^{n(t) \delta w} \mathcal{G}_{n(t) \delta w ; n(s) \nu v}(\sigma)
\end{gathered}
$$

where the matrices $\tilde{\mathcal{T}}$ in Eq. (4.20b) are the so-called rescaled twisted representation matrices 24]. Because of the Pauli matrices in $\mathcal{Y}$, it is not difficult to check that all these eigenfields have only two independent components in the two-dimensional space:

$$
\begin{gathered}
\mathcal{W}(X)_{n(r) \mu u}{ }^{n(s) \nu v}=\mathcal{W}^{(u-v)}(X)_{n(r) \mu}{ }^{n(s) \nu}, \quad \mathcal{E}(X)_{n(r) \mu u}{ }^{n(s) \nu v}=\mathcal{E}^{(u-v)}(X)_{n(r) \mu}{ }^{n(s) \nu} \\
\mathcal{G}_{n(r) \mu u ; n(s) \nu v}(X)=\mathcal{G}_{n(r) \mu ; n(s) \nu}^{(u+v)}(X), \quad \mathcal{B}_{n(r) \mu u ; n(s) \nu v}(X)=\mathcal{B}_{n(r) \mu ; n(s) \nu}^{(u+v)}(X) \\
\mathcal{H}_{n(r) \mu u ; n(s) \nu v ; n(t) \delta w}(X)=\mathcal{H}_{n(r) \mu ; n(s) \nu ; n(t) \delta}^{(u+v+w)}(X), \quad \bar{u}, \bar{v}, \bar{w} \in\{0,1\} .
\end{gathered}
$$

This fact is also apparent when the eigenfields are expressed in terms of the standard eigenfields 24] of space-time orbifold theory.

$$
\begin{gathered}
X_{\sigma}^{n(r) \mu u}(\xi)=\frac{1}{2}\left(X_{\sigma}^{n(r) \mu}(\xi)-(-1)^{u} X_{\sigma}^{n(r) \mu}(-\xi)\right) \\
\mathcal{W}(X(x))_{n(r) \mu u}{ }^{n(s) \nu v}=\frac{1}{2}\left(\mathcal{W}\left(x^{0}\right)_{n(r) \mu}^{n(s) \nu}+(-1)^{u-v} \mathcal{W}\left(x^{1}\right)_{n(r) \mu}{ }^{n(s) \nu}\right) \\
\mathcal{E}(X(x))_{n(r) \mu u}{ }^{n(s) \nu v}=\frac{1}{2}\left(\mathcal{E}\left(x^{0}\right)_{n(r) \mu}{ }^{n(s) \nu}+(-1)^{u-v} \mathcal{E}\left(x^{1}\right)_{n(r) \mu}{ }^{n(s) \nu}\right) \\
\mathcal{G}_{n(r) \mu u ; n(s) \nu v}(X(x))=\mathcal{G}_{n(r) \mu ; n(s) \nu}\left(x^{0}\right)+(-1)^{u+v} \mathcal{G}_{n(r) \mu ; n(s) \nu}\left(x^{1}\right) \\
\mathcal{B}_{n(r) \mu u ; n(s) \nu v}(X(x))=\mathcal{B}_{n(r) \mu ; n(s) \nu}\left(x^{0}\right)+(-1)^{u+v} \mathcal{B}_{n(r) \mu ; n(s) \nu}\left(x^{1}\right) \\
\mathcal{H}_{n(r) \mu u n(s) \nu v ; n(t) \delta w}(X(x))=\mathcal{H}_{n(r) \mu ; n(s) \nu ; n(t) \delta}\left(x^{0}\right)+(-1)^{u+v+w} \mathcal{H}_{n(r) \mu ; n(s) \nu ; n(t) \delta}\left(x^{1}\right)
\end{gathered}
$$




$$
\begin{aligned}
& X_{\sigma}^{n(r) \mu}(\xi) \equiv x^{i}(\xi) \chi(\sigma)_{n(r) \mu}^{-1} U^{\dagger}(\sigma)_{i}{ }^{n(r) \mu} \\
& \mathcal{W}(x)_{n(r) \mu}{ }^{n(s) \nu} \equiv \chi(\sigma)_{n(r) \mu} U(\sigma)_{n(r) \mu}{ }^{a} \Omega(x)_{a}{ }^{b} \chi(\sigma)_{n(s) \nu}^{-1} U^{\dagger}(\sigma)_{b}{ }^{n(s) \nu} \\
& \mathcal{E}(x)_{n(r) \mu}{ }^{n(s) \nu} \equiv \chi(\sigma)_{n(r) \mu} U(\sigma)_{n(r) \mu}{ }^{i} e(x)_{i}{ }^{a} \chi(\sigma)_{n(s) \nu}^{-1} U^{\dagger}(\sigma)_{a}{ }^{n(s) \nu} \\
& \mathcal{G}_{n(r) \mu ; n(s) \nu}(x) \equiv \chi(\sigma)_{n(r) \mu} \chi(\sigma)_{n(s) \nu} U(\sigma)_{n(r) \mu}{ }^{i} U(\sigma)_{n(s) \nu}{ }^{j} G_{i j}(x) \\
& \mathcal{B}_{n(r) \mu ; n(s) \nu}(x) \equiv \chi(\sigma)_{n(r) \mu} \chi(\sigma)_{n(s) \nu} U(\sigma)_{n(r) \mu}{ }^{i} U(\sigma)_{n(s) \nu}{ }^{j} B_{i j}(x) \\
& \mathcal{H}_{n(r) \mu ; n(s) \nu ; n(t) \delta}(x) \equiv \chi(\sigma)_{n(r) \mu} \chi(\sigma)_{n(s) \nu} \chi(\sigma)_{n(t) \delta} U(\sigma)_{n(r) \mu}{ }^{i} U(\sigma)_{n(s) \nu}{ }^{j} U(\sigma)_{n(t) \delta}{ }^{k} H_{i j k}(x)
\end{aligned}
$$

which are obtained by using Eq. (A.3C) to do the internal sums on the two-component indices $\dot{I}$ in Eq. (4.17).

Many of the eigenfields also diagonalize the behavior under world-sheet parity:

$$
\begin{gathered}
X_{\sigma}^{n(r) \mu u}(-\xi)=-X_{\sigma}^{n(r) \mu v}(\xi)\left(\tau_{3}\right)_{v}{ }^{u}=(-1)^{u+1} X_{\sigma}^{n(r) \mu u}(\xi) \\
\partial_{n(r) \mu u}(-\xi)=(-1)^{u+1} \partial_{n(r) \mu u}(\xi) \\
\mathcal{Y}_{n(r) \mu u}{ }^{n(s) \nu v}(X(-\xi))=(-1)^{u+v+1} \mathcal{Y}_{n(r) \mu u}{ }^{n(s) \nu v}(X(\xi)) \\
\mathcal{G}_{n(r) \mu u ; n(s) \nu v}(X(-\xi))=(-1)^{u+v} \mathcal{G}_{n(r) \mu u ; n(s) \nu v}(X(\xi)) \\
\mathcal{B}_{n(r) \mu u ; n(s) \nu v}(X(-\xi))=(-1)^{u+v+1} \mathcal{B}_{n(r) \mu u ; n(s) \nu v}(X(\xi)) \\
\mathcal{H}_{n(r) \mu u ; n(s) \nu v ; n(t) \delta w}(X(-\xi))=(-1)^{u+v+w} \mathcal{H}_{n(r) \mu u ; n(s) \nu v ; n(t) \delta w}(X(\xi)) .
\end{gathered}
$$

These relations follow from the corresponding world-sheet parities in Eq. (4.4), but the relations in (4.23d,e) also follow from (4.23c) and the explicit forms in Eq. (4.20). More complicated behavior is obtained for other eigenfields

$$
\begin{gathered}
\mathcal{W}(X(-\xi))_{n(r) \mu u}{ }^{n(s) \nu v}=(-1)^{u-v} \mathcal{W}^{-1}(X(\xi))_{n(r) \mu u}{ }^{n(s) \nu v} \\
\mathcal{E}(X(-\xi))_{n(r) \mu u}{ }^{n(s) \nu v}=(-1)^{u-v}(\mathcal{E}(X(\xi)) \mathcal{W}(X(\xi)))_{n(r) \mu u}{ }^{n(s) \nu v}
\end{gathered}
$$

where we have used Eqs. (4.4), (4.17e) and (4.17g).

Finally, we may reexpress the strip form (4.11a) of the WZW action in terms of the 
eigenfields:

$$
\begin{gathered}
S_{W Z W}^{\text {strip }}=\int d t \int_{0}^{\pi} d \xi \mathcal{L} \\
\mathcal{L}=\frac{1}{8 \pi}\left(\mathcal{G}_{n(r) \mu u ; n(s) \nu v}(X)+\mathcal{B}_{n(r) \mu u ; n(s) \nu v}(X)\right) \partial_{+} X^{n(r) \mu u} \partial_{-} X^{n(s) \nu v} \\
=\frac{1}{8 \pi}\left(\mathcal{G}_{n(r) \mu ; n(s) \nu}^{(u+v)}(X)+\mathcal{B}_{n(r) \mu ; n(s) \nu}^{(u+v)}(X)\right) \partial_{+} X_{\sigma}^{n(r) \mu u} \partial_{-} X_{\sigma}^{n(s) \nu v} \\
=\frac{1}{8 \pi} \sum_{w=0}^{1}\left(\mathcal{G}_{n(r) \mu ; n(s) \nu}^{(w)}(X)+\mathcal{B}_{n(r) \mu ; n(s) \nu}^{(w)}(X)\right) \sum_{u=0}^{1} \partial_{+} X^{n(r) \mu u} \partial_{-} X^{n(s) \nu, w-u}
\end{gathered}
$$

This form can also be obtained from the WZW action (3.7) on $\Gamma_{1 / 2}$ by using the identity (4.18c), the Gauss' law (4.18g) and Eq. (4.9).

\subsection{Einstein Geometry of the Twisted Open WZW Strings}

We now move to the twisted fields, using the principle of local isomorphisms [3, 5, 14, 24]

$$
\begin{gathered}
\mathcal{G} \underset{\sigma}{\longrightarrow} \hat{g}, \quad x \underset{\sigma}{\longrightarrow} \hat{x}, \quad \mathcal{W} \underset{\sigma}{\longrightarrow} \hat{\Omega}, \quad \mathcal{E} \underset{\sigma}{\longrightarrow} \hat{e} \\
\mathcal{G}, \quad \mathcal{B} \underset{\sigma}{\longrightarrow} \hat{B}, \quad \mathcal{H} \underset{\sigma}{\longrightarrow} \hat{H}, \quad \tilde{j} \underset{\sigma}{\longrightarrow} \hat{j} \\
S_{W Z W}^{\operatorname{strip}} \underset{\sigma}{\longrightarrow} \hat{S}_{\hat{g}_{O}(\sigma)}^{\operatorname{strip}}
\end{gathered}
$$

to map the eigenfields and the action to the twisted fields and the action formulation of the twisted open-string sector.

We begin with some useful relations among the twisted fields

$$
\begin{gathered}
\hat{x}_{\sigma}^{n(r) \mu u} \equiv \hat{x}_{\sigma}^{n(r) \mu u}(\xi, t), \quad \hat{\partial}_{n(r) \mu u}(\xi) \equiv \frac{\partial}{\partial \hat{x}^{n(r) \mu u}(\xi)}, \quad \bar{u}=0,1 \\
\hat{g}(\mathcal{T}, \hat{x}) \mathcal{T}_{n(r) \mu u} \hat{g}^{-1}(\mathcal{T}, \hat{x})=\hat{\Omega}(\hat{x})_{n(r) \mu u}{ }^{n(s) \nu v} \mathcal{T}_{n(s) \nu v} \\
\hat{e}_{n(r) \mu u}(\mathcal{T}, \hat{x})=-i \hat{g}^{-1}(\mathcal{T}, \hat{x}) \hat{\partial}_{n(r) \mu u} \hat{g}(\mathcal{T}, \hat{x})=\hat{e}(\hat{x})_{n(r) \mu u}{ }^{n(s) \nu v} \mathcal{T}_{n(s) \nu v} \\
\hat{e}(\hat{x})_{n(r) \mu u}{ }^{n(s) \nu v}=-i \widehat{T r}\left(\left(\mathcal{M} \otimes \mathbb{1}_{2}\right) \hat{g}^{-1}(\mathcal{T}, \hat{x}) \hat{\partial}_{n(r) \mu u} \hat{g}(\mathcal{T}, \hat{x}) \mathcal{G}^{n(s) \nu v ; n(t) \delta w}(\sigma) \mathcal{T}_{n(t) \delta w}\right)
\end{gathered}
$$




$$
\begin{gathered}
\hat{G}_{n(r) \mu u ; n(s) \nu v}(\hat{x})=-\widehat{\operatorname{Tr}}\left(\left(\mathcal{M} \otimes \mathbb{1}_{2}\right) \hat{g}^{-1} \hat{\partial}_{n(r) \mu u} \hat{g} \hat{g}^{-1} \hat{\partial}_{n(s) \nu v} \hat{g}\right) \\
=\hat{e}(\hat{x})_{n(r) \mu u}{ }^{n\left(r^{\prime}\right) \mu^{\prime} u^{\prime}} \hat{e}(\hat{x})_{n(s) \nu v} n\left(s^{\prime}\right) \nu^{\prime} v^{\prime} \mathcal{G}_{n\left(r^{\prime}\right) \mu^{\prime} u^{\prime} ; n\left(s^{\prime}\right) \nu^{\prime} v^{\prime}}(\sigma) \\
=\hat{e}(\hat{x})_{n(r) \mu u}{ }^{n\left(r^{\prime}\right) \mu^{\prime} u^{\prime}} \hat{e}(\hat{x})_{n(s) \nu v}{ }^{-n\left(r^{\prime}\right), \nu^{\prime},-u^{\prime}} \mathcal{G}_{n\left(r^{\prime}\right) \mu^{\prime} u^{\prime} ;-n\left(r^{\prime}\right), \nu^{\prime},-u^{\prime}}(\sigma) \\
\hat{H}_{n(r) \mu u ; n(s) \nu v ; n(t) \delta w}(\hat{x})=\hat{\partial}_{n(r) \mu u} \hat{B}_{n(s) \nu v ; n(t) \delta w}(\hat{x})+\operatorname{cyclic} \\
=\hat{e}_{n(r) \mu u}^{n\left(r^{\prime}\right) \mu^{\prime} u^{\prime}} \hat{e}_{n(s) \nu v}^{n\left(s^{\prime}\right) \nu^{\prime} v^{\prime}} \hat{e}_{n(t) \delta w}^{n\left(t^{\prime}\right) \delta^{\prime} w^{\prime}} \mathcal{F}_{n\left(r^{\prime}\right) \mu^{\prime} u^{\prime} ; n\left(s^{\prime}\right) \nu^{\prime} v^{\prime} ; n\left(t^{\prime}\right) \delta^{\prime} w^{\prime}}(\sigma) \\
\epsilon^{A B C} \widehat{\operatorname{Tr}}\left((\mathcal{M} \otimes \mathbb{1})\left(\hat{g}^{-1} \partial_{A} \hat{g} \hat{g}^{-1} \partial_{B} \hat{g} \hat{g}^{-1} \partial_{C} \hat{g}\right)\right)=\partial_{A} \hat{j}^{A}(\xi) \\
\hat{j}^{A}(\xi)=\frac{3}{2} \epsilon^{A B C} \partial_{B} \hat{x}_{\sigma}^{n(r) \mu u}(\xi) \partial_{C} \hat{x}_{\sigma}^{n(s) \nu v}(\xi) \hat{B}_{n(r) \mu u ; n(s) \nu v}(\hat{x}(\xi))
\end{gathered}
$$

which follow by local isomorphisms from the corresponding relations among the eigenfields. Here $\mathcal{G}^{\bullet}(\sigma)$ in (4.27d $)$ is the total inverse twisted metric defined in Eq. (3.20d), and we have used the selection rule (2.14a) for the tangent-space metric $\mathcal{G}_{\bullet}(\sigma)$ to obtain the reduced form (4.27f) of the twisted Einstein metric $\hat{G}(\hat{x})$. A similar reduced form of the twisted torsion $\hat{H}(\hat{x})$ can be obtained by using the selection rules $(2.13 \mathrm{a}, \mathrm{b})$, (4.18e). The quantity $\hat{j}^{A}(\xi)$ in Eq. (4.27i) will be called the twisted winding-number current.

From Eqs. (4.23), (4.24), the principle of local isomorphisms also gives us the worldsheet parities of the twisted fields:

$$
\begin{gathered}
\hat{x}_{\sigma}^{n(r) \mu u}(-\xi)=-\hat{x}_{\sigma}^{n(r) \mu v}(\xi)\left(\tau_{3}\right)_{v}^{u}=(-1)^{u+1} \hat{x}_{\sigma}^{n(r) \mu u}(\xi) \\
\hat{\partial}_{n(r) \mu u}(-\xi)=(-1)^{u+1} \hat{\partial}_{n(r) \mu u}(\xi) \\
\hat{\Omega}(\hat{x}(-\xi))_{n(r) \mu u}{ }^{n(s) \nu v}=(-1)^{u-v} \hat{\Omega}^{-1}(\hat{x}(\xi))_{n(r) \mu u} n(s) \nu v \\
\hat{e}(\hat{x}(-\xi))_{n(r) \mu u}{ }^{n(s) \nu v}=(-1)^{u-v}(\hat{e}(\hat{x}(\xi)) \hat{\Omega}(\hat{x}(\xi)))_{n(r) \mu u}{ }^{n(s) \nu v} \\
\hat{G}_{n(r) \mu u ; n(s) \nu v}(\hat{x}(-\xi))=(-1)^{u+v} \hat{G}_{n(r) \mu u ; n(s) \nu v}(\hat{x}(\xi)) \\
\hat{B}_{n(r) \mu u ; n(s) \nu v}(\hat{x}(-\xi))=(-1)^{u+v+1} \hat{B}_{n(r) \mu u ; n(s) \nu v}(\hat{x}(\xi)) \\
\hat{H}_{n(r) \mu u ; n(s) \nu v ; n(t) \delta w}(\hat{x}(-\xi))=(-1)^{u+v+w} \hat{H}_{n(r) \mu u ; n(s) \nu v ; n(t) \delta w}(\hat{x}(\xi)) \\
\hat{j} \xi(-\xi)=-\hat{j}^{\xi}(\xi), \quad \hat{j}^{t, \rho}(-\xi)=\hat{j}^{t, \rho}(\xi) .
\end{gathered}
$$

We also give the explicit functional forms

$$
\begin{gathered}
\hat{\Omega}(\hat{x})=e^{-i \hat{Y}(\hat{x})}, \quad \hat{Y}(\hat{x}) \equiv \hat{x}_{\sigma}^{n(r) \mu u} \tilde{\mathcal{T}}_{n(r) \mu}^{a d j} \tau_{u} \\
\hat{e}(\hat{x})_{n(r) \mu u}^{n(s) \nu v}=\left(\frac{e^{i \hat{Y}(\hat{x})-1}}{i \hat{Y}(\hat{x})}\right)_{n(r) \mu u}^{n(s) \nu v}, \hat{e}(0)_{n(r) \mu u}^{n(s) \nu v}=\delta_{\mu}^{\nu} \delta_{n(r)+n(s), 0 \bmod \rho(\sigma)} \delta_{u+v, 0 \bmod 2}
\end{gathered}
$$




$$
\begin{aligned}
& \hat{G}_{n(r) \mu u ; n(s) \nu v}(\hat{x})=\left(\frac{e^{i \hat{Y}(\hat{x})+e^{-i \hat{Y}(\hat{x})}-2}}{(i \hat{Y}(\hat{x}))^{2}}\right)_{n(r) \mu u}^{n(t) \delta w} \mathcal{G}_{n(t) \delta w ; n(s) \nu v}(\sigma) \\
& =\left(\frac{e^{i \hat{Y}(\hat{x})+e^{-i \hat{Y}(\hat{x})}-2}}{(i \hat{Y}(\hat{x}))^{2}}\right)_{n(s) \nu v}^{n(t) \delta w} \mathcal{G}_{n(t) \delta w ; n(r) \mu u}(\sigma) \\
& =\left(\frac{e^{i \hat{Y}(\hat{x})}+e^{-i \hat{Y}(\hat{x})}-2}{(i \hat{Y}(\hat{x}))^{2}}\right)_{n(r) \mu u}^{-n(s), \nu,-v} \mathcal{G}_{-n(s), \nu,-v ; n(s) \nu v}(\sigma) \\
& \hat{B}_{n(r) \mu u ; n(s) \nu v}(\hat{x})=\left(\frac{e^{i \hat{Y}(\hat{x})}-e^{-i \hat{Y}(\hat{x})}-2 i \hat{Y}(\hat{x})}{(i \hat{Y}(\hat{x}))^{2}}\right)_{n(r) \mu u}^{n(t) \delta w} \mathcal{G}_{n(t) \delta w ; n(s) \nu v}(\sigma) \\
& =-\left(\frac{e^{i \hat{Y}(\hat{x})}-e^{-i \hat{Y}(\hat{x})}-2 i \hat{Y}(\hat{x})}{(i \hat{Y}(\hat{x}))^{2}}\right)_{n(s) \nu v}^{n(t) \delta w} \mathcal{G}_{n(t) \delta w ; n(r) \mu u}(\sigma) \\
& =\left(\frac{e^{i \hat{Y}(\hat{x})}-e^{-i \hat{Y}(\hat{x})}-2 i \hat{Y}(\hat{x})}{(i \hat{Y}(\hat{x}))^{2}}\right)_{n(r) \mu u}^{-n(s), \nu,-v} \mathcal{G}_{-n(s), \nu,-v ; n(s) \nu v}(\sigma)
\end{aligned}
$$

which follow by local isomorphisms from Eq. (4.20). The rescaled matrices $\tilde{\mathcal{T}}^{\text {adj }}$ which appear in these formulae were defined in Eq. (4.20b).

Like the group orbifold elements in Eq. (2.23) and the twisted Einstein coordinates (4.27a), the explicit functional forms in Eq. (4.29) tell us that the other twisted fields are also two-component fields

$$
\begin{gathered}
\hat{\Omega}(\hat{x})_{n(r) \mu u}^{n(s) \nu v}=\hat{\Omega}^{(u-v)}(\hat{x})_{n(r) \mu}^{n(s) \nu}, \quad \hat{\Omega}^{-1}(\hat{x})_{n(r) \mu u}^{n(s) \nu v}=\hat{\Omega}_{(u-v)}^{-1}(\hat{x})_{n(r) \mu}^{n(s) \nu} \\
\hat{e}(\hat{x})_{n(r) \mu u}{ }^{n(s) \nu v}=\hat{e}^{(u-v)}(\hat{x})_{n(r) \mu} n(s) \nu \\
\hat{G}_{n(r) \mu u ; n(s) \nu v}(\hat{x})=\hat{G}_{n(r) \mu ; n(s) \nu}^{(u+v)}(\hat{x}), \quad \hat{B}_{n(r) \mu u ; n(s) \nu v}(\hat{x})=\hat{B}_{n(r) \mu ; n(s) \nu}^{(u+v)}(\hat{x}) \\
\hat{H}_{n(r) \mu u ; n(s) \nu v ; n(t) \delta w}(\hat{x})=\hat{H}_{n(r) \mu ; n(s) \nu ; n(t) \delta}^{(u+v+w)}(\hat{x}), \quad \bar{u}, \bar{v}, \bar{w} \in\{0,1\}
\end{gathered}
$$

and all of the relations above simplify accordingly. For example, the following world-sheet parities of the reduced components

$$
\begin{aligned}
& \hat{\Omega}^{(w)}(\hat{x}(-\xi))_{n(r) \mu}{ }^{n(s) \nu}=(-1)^{w} \hat{\Omega}_{(w)}^{-1}(\hat{x}(\xi))_{n(r) \mu}{ }^{n(s) \nu} \\
& \hat{e}^{(w)}(\hat{x}(-\xi))_{n(r) \mu}{ }^{n(s) \nu}=(-1)^{w} \sum_{v=0}^{1} \hat{e}^{(v)}(\hat{x}(\xi))_{n(r) \mu}{ }^{n(t) \delta} \hat{\Omega}^{(w-v)}(\hat{x}(\xi))_{n(t) \delta} n(s) \nu \\
& \hat{G}_{n(r) \mu ; n(s) \nu}^{(w)}(\hat{x}(-\xi))=(-1)^{w} \hat{G}_{n(r) \mu ; n(s) \nu}^{(w)}(\hat{x}(\xi)) \\
& \hat{B}_{n(r) \mu ; n(s) \nu}^{(w)}(\hat{x}(-\xi))=(-1)^{w+1} \hat{B}_{n(r) \mu ; n(s) \nu}^{(w)}(\hat{x}(\xi)) \\
& \hat{H}_{n(r) \mu ; n(s) \nu ; n(t) \delta}^{(w)}(\hat{x}(-\xi))=(-1)^{w} \hat{H}_{n(r) \mu ; n(s) \nu ; n(t) \delta}^{(w)}(\hat{x}(\xi))
\end{aligned}
$$

are obtained by substitution of the reduced forms (4.30) into Eq. (4.28). 
The open-string sigma-model form of the WZW orientation-orbifold action can be obtained by derived local isomorphisms from the (eigenfield) sigma-model form (4.25) of the WZW action:

$$
\begin{gathered}
\hat{S}_{\hat{g}_{O}(\sigma)}^{\text {strip }} \equiv \int d t \int_{0}^{\pi} d \xi \hat{\mathcal{L}}_{\sigma}^{W Z W} \\
\hat{\mathcal{L}}_{\sigma}^{W Z W}=\frac{1}{8 \pi}\left(\hat{G}_{n(r) \mu u ; n(s) \nu v}(\hat{x})+\hat{B}_{n(r) \mu u ; n(s) \nu v}(\hat{x})\right) \partial_{+} \hat{x}_{\sigma}^{n(r) \mu u} \partial_{-} \hat{x}_{\sigma}^{n(s) \nu v} \\
=\frac{1}{8 \pi}\left(\hat{G}_{n(r) \mu ; n(s) \nu}^{(u+v)}(\hat{x})+\hat{B}_{n(r) \mu ; n(s) \nu}^{(u+v)}(\hat{x})\right) \partial_{+} \hat{x}_{\sigma}^{n(r) \mu u} \partial_{-} \hat{x}_{\sigma}^{n(s) \nu v} \\
=\frac{1}{8 \pi} \sum_{w=0}^{1}\left(\hat{G}_{n(r) \mu ; n(s) \nu}^{(w)}(\hat{x})+\hat{B}_{n(r) \mu ; n(s) \nu}^{(w)}(\hat{x})\right) \sum_{u=0}^{1} \partial_{+} \hat{x}_{\sigma}^{n(r) \mu u} \partial_{-} \hat{x}_{\sigma}^{n(s) \nu, w-u} \\
\hat{S}_{\hat{g}_{O}(\sigma)}^{\text {strip }}=\hat{S}_{\hat{g}_{O}(\sigma)}\left[\mathcal{M} \otimes \mathbb{1}_{2}, \hat{g} ; \Gamma_{\frac{1}{2}}\right] .
\end{gathered}
$$

We finally comment on the equivalence (4.32c) of the WZW orientation-orbifold action (3.9) on $\Gamma_{1 / 2}$ and this open-string sigma-model form on the strip, both of which followed above for all $\hat{h}_{\sigma}$ by local isomorphisms - without the use of monodromy. Using the twisted Gauss' law (4.27h) and the form of the twisted Einstein metric in Eq. (4.27e) to compare these two actions, we find the $(3 \leftrightarrow 2)$-dimensional consistency relation

$$
\begin{gathered}
\int_{\Gamma_{\frac{1}{2}}} \widehat{\operatorname{Tr}}\left(\left(\mathcal{M} \otimes \mathbb{1}_{2}\right)\left(\hat{g}^{-1}(\mathcal{T}, \xi) d \hat{g}(\mathcal{T}, \xi)\right)^{3}\right) \\
=-\frac{3}{2} \int d t \int_{0}^{\pi} d \xi \hat{B}_{n(r) \mu u ; n(s) \nu v}(\hat{x}) \partial_{+} \hat{x}^{n(r) \mu u} \partial_{-} \hat{x}^{n(s) \nu v} \\
\Rightarrow \int_{y=0} d t d x\left(-\vec{e}_{y} \cdot \hat{j}\right)=\int_{y=0} d t d x\left(\hat{j}^{\xi}(\pi)-\hat{j}^{\xi}(0)\right)=0
\end{gathered}
$$

which requires (as in Subsec. 4.2) a vanishing contribution from the flat side of the solid half cylinder for all $\hat{h}_{\sigma}$. The local form of this boundary condition on the twisted windingnumber current

$$
\hat{j}^{\xi}(0)=\hat{j}^{\xi}(\pi)=0
$$

is verified explicitly for the case $h_{\sigma}^{2}=1$ in the following subsection. Another set of boundary conditions can be obtained in the usual fashion by variation of the open-string sigma-model form (4.32) of the action, but we will discuss these variational boundary conditions more generally in Subsec. 5.2. 


\subsection{Monodromies and Extra Boundary Conditions}

As above, we can obtain further structure in the case $h_{\sigma}^{2}=1$, for which local isomorphisms gives us the monodromies of all the twisted open-string fields:

$$
\begin{gathered}
h_{\sigma}^{2}=1: \quad \text { automorphic responses } \underset{\sigma}{\longrightarrow} \text { monodromies } \\
\hat{g}(\mathcal{T}, \hat{x}(\xi+2 \pi))=\tau_{3} E(T, \sigma) \hat{g}(\mathcal{T}, \hat{x}(\xi)) E^{*}(T, \sigma) \tau_{3} \\
\hat{x}_{\sigma}^{n(r) \mu u}(\xi+2 \pi)=\hat{x}_{\sigma}^{n(r) \mu u}(\xi) e^{2 \pi i\left(\frac{n(r)}{\rho(\sigma)}+\frac{u}{2}\right)}, \quad \bar{u}=0,1 \\
\hat{\partial}_{n(r) \mu u}(\xi) \equiv \frac{\partial}{\partial \hat{x}^{n(r) \mu u}(\xi)}, \quad \hat{\partial}_{n(r) \mu u}(\xi+2 \pi)=e^{-2 \pi i\left(\frac{n(r)}{\rho(\sigma)}+\frac{u}{2}\right)} \hat{\partial}_{n(r) \mu u}(\xi) \\
\hat{\partial}_{n(r) \mu u}(\xi) \hat{x}_{\sigma}^{n(s) \nu v}(\xi)=\delta_{\mu}^{\nu} \delta_{n(r)-n(s), 0 \bmod \rho(\sigma)} \delta_{u-v, 0 \bmod 2} \\
\hat{\Omega}(\hat{x}(\xi+2 \pi))_{n(r) \mu u}{ }^{n(s) \nu v}=e^{-2 \pi i\left(\frac{n(r)-n(s)}{\rho(\sigma)}+\frac{u-v}{2}\right)} \hat{\Omega}(\hat{x}(\xi))_{n(r) \mu u} n(s) \nu v \\
\hat{e}_{n(r) \mu u}(\mathcal{T}, \hat{x}(\xi+2 \pi))=e^{-2 \pi i\left(\frac{n(r)}{\rho(\sigma)}+\frac{u}{2}\right)} \tau_{3} E(T, \sigma) \hat{e}_{n(r) \mu u}(\mathcal{T}, \hat{x}(\xi)) E^{*}(T, \sigma) \tau_{3} \\
\hat{e}(\hat{x}(\xi+2 \pi))_{n(r) \mu u}{ }^{n(s) \nu v}=e^{-2 \pi i\left(\frac{n(r)-n(s)}{\rho(\sigma)}+\frac{u-v}{2}\right)} \hat{e}(\hat{x}(\xi))_{n(r) \mu u} n(s) \nu v \\
\hat{G}_{n(r) \mu u ; n(s) \nu v}(\hat{x}(\xi+2 \pi))=e^{-2 \pi i\left(\frac{n(r)+n(s)}{\rho(\sigma)}+\frac{u+v}{2}\right)} \hat{G}_{n(r) \mu u ; n(s) \nu v}(\hat{x}(\xi)) \\
\hat{B}_{n(r) \mu u ; n(s) \nu v}(\hat{x}(\xi+2 \pi))=e^{-2 \pi i\left(\frac{n(r)+n(s)}{\rho(\sigma)}+\frac{u+v}{2}\right)} \hat{B}_{n(r) \mu u ; n(s) \nu v}(\hat{x}(\xi)) \\
\hat{H}_{n(r) \mu u ; n(s) \nu v ; n(t) \delta w}(\hat{x}(\xi+2 \pi))=e^{-2 \pi i\left(\frac{n(r)+n(s)+n(t)}{\rho(\sigma)}+\frac{u+v+w}{2}\right)} \hat{H}_{n(r) \mu u ; n(s) \nu v ; n(t) \delta w}(\hat{x}(\xi)) \\
\hat{j}^{A}(\xi+2 \pi)=\hat{j}^{A}(\xi) \\
\hat{\mathcal{L}}_{\sigma}^{W Z W}(\hat{x}(\xi+2 \pi))=\hat{\mathcal{L}}_{\sigma}^{W Z W}(\hat{x}(\xi))
\end{gathered}
$$

The restriction (4.35a) to $h_{\sigma}^{2}=1$ follows from the discussion of Subsec. 3.2 (and in particular, from Eq. (2.29) ) and our choice of coordinate system $x=\beta$ in Eq. (4.2).

For the reduced components $\hat{A}^{(w)}$ of the twisted fields, one finds that the monodromies

$$
\begin{aligned}
& \hat{\Omega}^{(w)}(\hat{x}(\xi+2 \pi))_{n(r) \mu}{ }^{n(s) \nu}=e^{-2 \pi i\left(\frac{n(r)-n(s)}{\rho(\sigma)}+\frac{w}{2}\right)} \hat{\Omega}^{(w)}(\hat{x}(\xi))_{n(r) \mu}{ }^{n(s) \nu} \\
& \hat{e}^{(w)}(\hat{x}(\xi+2 \pi))_{n(r) \mu} n(s) \nu=e^{-2 \pi i\left(\frac{n(r)-n(s)}{\rho(\sigma)}+\frac{w}{2}\right)} \hat{e}^{(w)}(\hat{x}(\xi))_{n(r) \mu}{ }^{n(s) \nu} \\
& \hat{G}_{n(r) \mu ; n(s) \nu}^{(w)}(\hat{x}(\xi+2 \pi))=e^{-2 \pi i\left(\frac{n(r)+n(s)}{\rho(\sigma)}+\frac{w}{2}\right)} \hat{G}_{n(r) \mu ; n(s) \nu}^{(w)}(\hat{x}(\xi)) \\
& \hat{B}_{n(r) \mu ; n(s) \nu}^{(w)}(\hat{x}(\xi+2 \pi))=e^{-2 \pi i\left(\frac{n(r)+n(s)}{\rho(\sigma)}+\frac{w}{2}\right)} \hat{B}_{n(r) \mu ; n(s) \nu}^{(w)}(\hat{x}(\xi)) \\
& \hat{H}_{n(r) \mu ; n(s) \nu ; n(t) \delta}^{(w)}(\hat{x}(\xi+2 \pi))=e^{-2 \pi i\left(\frac{n(r)+n(s)+n(t)}{\rho(\sigma)}+\frac{w}{2}\right)} \hat{H}_{n(r) \mu ; n(s) \nu ; n(t) \delta}^{(w)}(\hat{x}(\xi))
\end{aligned}
$$


follow from Eqs. (4.35) and (4.30).

Then following steps analogous to those given in Eq. (3.30), the monodromies (4.35c), (4.36) and the world-sheet parities (4.28a), (4.31) give the boundary conditions for the twisted fields on the strip:

$$
\begin{aligned}
& \hat{x}_{\sigma}^{n(r) \mu 0}(0)=0, \quad \hat{x}_{\sigma}^{n(r) \mu u}(\pi)=0 \text { unless } \frac{n(r)}{\rho(\sigma)} \in \mathbb{Z}+\frac{1}{2} \\
& \left(\partial_{t} \hat{x}_{\sigma}^{n(r) \mu 0}\right)(0)=0, \quad\left(\partial_{t} \hat{x}_{\sigma}^{n(r) \mu u}\right)(\pi)=0 \text { unless } \frac{n(r)}{\rho(\sigma)} \in \mathbb{Z}+\frac{1}{2} \\
& \left(\partial_{\xi} \hat{x}_{\sigma}^{n(r) \mu 1}\right)(0)=0, \quad\left(\partial_{\xi} \hat{x}_{\sigma}^{n(r) \mu u}\right)(\pi)=0 \text { unless } \frac{n(r)}{\rho(\sigma)} \in \mathbb{Z} \\
& {\left[\hat{\Omega}^{(0)}(\hat{x}), \hat{\Omega}^{(1)}(\hat{x})\right]=0, \quad \hat{\Omega}^{(0)}(\hat{x})^{2}-\hat{\Omega}^{(1)}(\hat{x})^{2}=\mathbb{1} \text { at } \xi=0} \\
& \left.\begin{array}{l}
\hat{\Omega}^{(0)}(\hat{x}) E(\sigma) \hat{\Omega}^{(0)}(\hat{x})+\hat{\Omega}^{(1)}(\hat{x}) E(\sigma) \hat{\Omega}^{(1)}(\hat{x})=E(\sigma) \\
\hat{\Omega}^{(0)}(\hat{x}) E(\sigma) \hat{\Omega}^{(1)}(\hat{x})+\hat{\Omega}^{(1)}(\hat{x}) E(\sigma) \hat{\Omega}^{(0)}(\hat{x})= \\
0
\end{array}\right\} \text { at } \xi=\pi \\
& \sum_{v=0}^{1} \hat{e}^{(v)}(\hat{x}(0))_{n(r) \mu}{ }^{n(t) \delta}\left(\delta_{v}^{w} \delta_{n(t) \delta}^{n(s) \nu}-(-1)^{w} \hat{\Omega}^{(w-v)}(\hat{x}(0))_{n(t) \delta} n(s) \nu\right)=0 \\
& \sum_{v=0}^{1} \hat{e}^{(v)}(\hat{x}(\pi))_{n(r) \mu}{ }^{n(t) \delta}\left(\delta_{v}^{w} \delta_{n(t) \delta}^{n(s) \nu} e^{2 \pi i\left(\frac{n(r)-n(t)}{\rho(\sigma)}\right)}-\hat{\Omega}^{(w-v)}(\hat{x}(\pi))_{n(t) \delta}{ }^{n(s) \nu}\right)=0 \\
& \hat{G}_{n(r) \mu ; n(s) \nu}^{(1)}(\hat{x}(0))=0, \quad \hat{G}_{n(r) \mu ; n(s) \nu}^{(w)}(\hat{x}(\pi))=0 \text { unless } \frac{n(r)+n(s)}{\rho(\sigma)} \in \mathbb{Z} \\
& \left(\partial_{\xi} \hat{G}_{n(r) \mu ; n(s) \nu}^{(0)}\right)(\hat{x}(0))=0, \quad\left(\partial_{\xi} \hat{G}_{n(r) \mu ; n(s) \nu}^{(w)}\right)(\hat{x}(\pi))=0 \text { unless } \frac{n(r)+n(s)}{\rho(\sigma)} \in \mathbb{Z}+\frac{1}{2} \\
& \hat{B}_{n(r) \mu ; n(s) \nu}^{(0)}(\hat{x}(0))=0, \quad \hat{B}_{n(r) \mu ; n(s) \nu}^{(w)}(\hat{x}(\pi))=0 \text { unless } \frac{n(r)+n(s)}{\rho(\sigma)} \in \mathbb{Z}+\frac{1}{2} \\
& \left(\partial_{\xi} \hat{B}_{n(r) \mu ; n(s) \nu}^{(1)}\right)(\hat{x}(0))=0, \quad\left(\partial_{\xi} \hat{B}_{n(r) \mu ; n(s) \nu}^{(w)}\right)(\hat{x}(\pi))=0 \text { unless } \frac{n(r)+n(s)}{\rho(\sigma)} \in \mathbb{Z} \\
& \hat{H}_{n(r) \mu ; n(s) \nu ; n(t) \delta}^{(1)}(\hat{x}(0))=0, \quad \hat{H}_{n(r) \mu ; n(s) \nu ; n(t) \delta}^{(w)}(\hat{x}(\pi))=0 \text { unless } \frac{n(r)+n(s)+n(t)}{\rho(\sigma)} \in \mathbb{Z} \\
& \left(\partial_{\xi} \hat{H}_{n(r) \mu ; n(s) \nu ; n(t) \delta}^{(0)}\right)(\hat{x}(0))=0 \\
& \left(\partial_{\xi} \hat{H}_{n(r) \mu ; n(s) \nu ; n(t) \delta}^{(w)}\right)(\hat{x}(\pi))=0 \text { unless } \frac{n(r)+n(s)+n(t)}{\rho(\sigma)} \in \mathbb{Z}+\frac{1}{2} .
\end{aligned}
$$

The eigenvalue matrix $E(\sigma)$ which appears in Eq. (4.37e) was defined in Eq. (2.11). Here we gave the $\partial_{t}$ boundary conditions explicitly for $\hat{x}$ only, but the $\partial_{t}$ boundary conditions for $\hat{G}, \hat{B}$ and $\hat{H}$ are also the same as those for the fields themselves. 
We also remark that the trivial monodromy (4.351) and the world-sheet parity (4.28h) of the twisted winding-number current $\hat{j}$ show that the $(3 \leftrightarrow 2)$-dimensional consistency relation (4.33b) is satisfied explicitly

$$
\text { monodromy }+ \text { world-sheet parity } \Rightarrow \hat{j}^{\xi}(0)=\hat{j}^{\xi}(\pi)=0
$$

in parallel with the discussion of the untwisted case in Subsec. 4.1.

As noted above, all the boundary conditions of this subsection at $\xi=\pi$ are true only for $h_{\sigma}^{2}=1$, while all the boundary conditions at $\xi=0$ follow from the world-sheet parity alone - and hence are valid for all $\hat{h}_{\sigma}$. Moreover, the boundary conditions of this subsection should be considered as further substructure of the WZW orientation-orbifold branes.

\section{$5 \quad$ Sigma-Model Orientation Orbifolds}

\subsection{Sigma Models with Orientation-Reversing Symmetry}

We consider here the nonlinear sigma model $A_{M}$ on a general target-space manifold $M$ with local ${ }^{\ddagger 6}$ Einstein coordinates $x^{i}, i=1 \ldots \operatorname{dim} M$ :

$$
\begin{gathered}
S=\frac{1}{8 \pi} \int d t \int_{0}^{2 \pi} d \xi\left(G_{i j}(x)+B_{i j}(x)\right) \partial_{+} x^{i} \partial_{-} x^{j}, \quad \partial_{ \pm}=\partial_{t} \pm \partial_{\xi} \\
H_{i j k}(x)=\partial_{i} B_{j k}(x)+\partial_{j} B_{k i}(x)+\partial_{k} B_{i j}(x), \quad x^{i}(\xi+2 \pi)=x^{i}(\xi) .
\end{gathered}
$$

We begin by requiring that the sigma model action is invariant under the basic orientationreversing automorphism:

$$
\begin{gathered}
\hat{h}_{\sigma}=\tau_{1} \times \mathbb{1} \\
x^{i}(\xi)^{\prime}=-x^{i}(-\xi), \quad G_{i j}(x)^{\prime}=G_{i j}\left(x^{\prime}\right), \quad B_{i j}(x)^{\prime}=B_{i j}\left(x^{\prime}\right), \quad H_{i j k}(x)^{\prime}=H_{i j k}\left(x^{\prime}\right) .
\end{gathered}
$$

Then one finds

$$
\begin{aligned}
S^{\prime} & =\frac{1}{8 \pi} \int d t \int_{0}^{2 \pi} d \xi\left(G_{i j}(-x(-\xi))+B_{i j}(-x(-\xi))\right) \partial_{+} x^{i}(-\xi) \partial_{-} x^{j}(-\xi) \\
& =\frac{1}{8 \pi} \int d t \int_{-2 \pi}^{0} d \xi\left(G_{i j}(-x(\xi))+B_{i j}(-x(\xi))\right) \partial_{-} x^{i}(\xi) \partial_{+} x^{j}(\xi)
\end{aligned}
$$

\footnotetext{
${ }^{\ddagger 6} \mathrm{~A}$ complete treatment of the nonlinear sigma model should include discussion of global issues (in which the discussion of the text pertains to a coordinate patch), but we will not do so here.
} 


$$
\begin{aligned}
& =\frac{1}{8 \pi} \int d t \int_{0}^{2 \pi} d \xi\left(G_{i j}(-x(\xi))+B_{i j}(-x(\xi))\right) \partial_{-} x^{i}(\xi) \partial_{+} x^{j}(\xi) \\
& =\frac{1}{8 \pi} \int d t \int_{0}^{2 \pi} d \xi\left(G_{i j}(-x(\xi))-B_{i j}(-x(\xi))\right) \partial_{+} x^{i}(\xi) \partial_{-} x^{j}(\xi) \equiv S
\end{aligned}
$$

where we needed the space-time parities

$$
G_{i j}(-x)=G_{i j}(x), \quad B_{i j}(-x)=-B_{i j}(x) \longrightarrow H_{i j k}(-x)=H_{i j k}(x)
$$

in the last step to obtain the invariance $S^{\prime}=S$. It follows that the space-time parity (5.4) is a necessary and sufficient condition for invariance of the sigma model under the basic orientation-reversing automorphism. In other words, orientation reversal is in the automorphism group of the isometry group of the manifold $\mathrm{M}$

$$
\tau_{1} \times \mathbb{1} \in A u t(\operatorname{Iso} M)
$$

iff M satisfies the space-time parities in Eq. (5.4).

We recall that the space-time parity (5.4) was verified explicitly in Subsec. 5.1 for the special case of the general WZW model, and hence follows as well for any principal chiral model. We have also checked (see App. B) that the space-time parity (5.4) holds for all reductive $g / h$ coset conformal field theories. In what follows, our discussion is restricted to such orientation-symmetric sigma models, although the classification of these models is beyond the scope of this paper.

As discussed above for WZW, the following two-component form of the sigma model on the strip

$$
\begin{gathered}
S=\int d t \int_{0}^{\pi} d \xi \mathcal{L} \\
\mathcal{L}=\frac{1}{8 \pi}\left(G_{i \dot{I} ; j \dot{j}}(x)+B_{i \dot{I} ; j \dot{J}}(x)\right) \partial_{+} x^{i \dot{I}} \partial_{-} x^{j \dot{J}} \\
x^{i \dot{I}}(\xi) \equiv(-1)^{\dot{I}} x^{i}\left((-1)^{\dot{I}} \xi\right), \quad x^{i \dot{I}}(\xi+2 \pi)=x^{i \dot{I}}(\xi) \\
G_{i \dot{I} ; j \dot{J}}(x) \equiv \delta_{\dot{I} \dot{J}} G_{i j}\left(x^{\dot{I}}\right), \quad B_{i \dot{I} ; j \dot{J}}(x) \equiv \delta_{\dot{I} \dot{J}} B_{i j}\left(x^{\dot{I}}\right) \\
H_{i \dot{I} ; j \dot{J} ; k \dot{K}}(x) \equiv \partial_{i \dot{I}} B_{j \dot{j} ; k \dot{K}}(x)+\operatorname{cyclic}=\delta_{\dot{I} \dot{j}} \delta_{\dot{j} \dot{K}} H_{i j k}\left(x^{\dot{I}}\right), \quad \dot{I}, \dot{J}, \dot{K} \in\{0,1\}
\end{gathered}
$$

is an equivalent form of the sigma model if and only if the space-time parity (5.4) holds.

Using the space-time parities in Eq. (5.4) and the definitions in Eq. (5.6), we also find 
the world-sheet parities of all the two-component fields of the sigma model (5.6b):

$$
\begin{gathered}
x^{i \dot{I}}(-\xi)=-x^{i \dot{J}}(\xi)\left(\tau_{1}\right)_{\dot{J}} \dot{I}, \quad \partial_{i \dot{I}}(-\xi)=-\left(\tau_{1}\right)_{\dot{I}}^{\dot{J}} \partial_{i \dot{J}}(\xi) \\
G_{i \dot{I} ; j \dot{J}}(x(-\xi))=\left(\tau_{1}\right)_{\dot{I}}^{\dot{K}}\left(\tau_{1}\right)_{\dot{J}}^{\dot{L}} G_{i \dot{K} ; j \dot{L}}(x(\xi)) \\
H_{i \dot{I} ; j \dot{J} ; k \dot{K}}(x(-\xi))=\left(\tau_{1}\right)_{\dot{I}}^{\dot{L}}\left(\tau_{1}\right)_{\dot{j}}^{\dot{M}}\left(\tau_{1}\right)_{\dot{K}}^{\dot{N}} H_{i \dot{L} ; j \dot{M} ; k \dot{N}}(x(\xi)) \\
B_{i \dot{I} ; j \dot{j}}(x(-\xi))=-\left(\tau_{1}\right)_{\dot{I}}^{\dot{K}}\left(\tau_{1}\right)_{\dot{j}}^{\dot{L}} B_{i \dot{K} ; j \dot{L}}(x(\xi)) .
\end{gathered}
$$

These forms are the same as those found above (see Eq. (4.4) for the corresponding twocomponent WZW fields.

In the two-component notation of Eq. (5.6), the basic orientation-reversing automorphism (5.2b) can be written as

$$
\begin{gathered}
x^{i \dot{I}}(\xi)^{\prime}=x^{i \dot{J}}(\xi)\left(\tau_{1}\right)_{\dot{j}}^{\dot{I}} \\
G_{i \dot{I} ; j \dot{J}}(x)^{\prime}=G_{i \dot{I} ; j \dot{J}}\left(x^{\prime}\right)=\left(\tau_{1}\right)_{\dot{I}}^{\dot{K}}\left(\tau_{1}\right)_{\dot{j}}^{\dot{L}} G_{i \dot{K} ; j \dot{L}}(x) \\
B_{i \dot{I} ; j \dot{J}}(x)^{\prime}=B_{i \dot{I} ; j \dot{J}}\left(x^{\prime}\right)=\left(\tau_{1}\right)_{\dot{I}}^{\dot{K}}\left(\tau_{1}\right)_{\dot{j}}^{\dot{L}} B_{i \dot{K} ; j \dot{L}}(x) \\
H_{i \dot{I} ; j \dot{j} ; k \dot{K}}(x)^{\prime}=H_{i \dot{I} ; j \dot{j} ; k \dot{K}}\left(x^{\prime}\right)=\left(\tau_{1}\right)_{\dot{I}}^{\dot{L}}\left(\tau_{1}\right)_{\dot{J}}^{\dot{M}}\left(\tau_{1}\right)_{\dot{K}}{ }^{\dot{N}} H_{i \dot{L} ; j \dot{M} ; k \dot{N}}(x)
\end{gathered}
$$

and the invariance of the strip action (5.6) under (5.8) is transparent.

We may further require that the more general orientation reversal

$$
\begin{gathered}
x^{i \dot{I}}(\xi)^{\prime}=x^{j \dot{J}}(\xi) \omega^{\dagger}\left(h_{\sigma}\right)_{j}{ }^{i}\left(\tau_{1}\right)_{\dot{j}}{ }^{\dot{I}} \\
G_{i \dot{I} ; j \dot{J}}(x)^{\prime}=G_{i \dot{I} ; j \dot{j}}\left(x^{\prime}\right)=\omega\left(h_{\sigma}\right)_{i}{ }^{k} \omega\left(h_{\sigma}\right)_{j}{ }^{l}\left(\tau_{1}\right)_{\dot{I}}{ }^{\dot{K}}\left(\tau_{1}\right)_{\dot{J}}{ }^{\dot{L}} G_{k \dot{K} ; l \dot{L}}(x) \\
B_{i \dot{I} ; j \dot{j}}(x)^{\prime}=B_{i \dot{I} ; j \dot{J}}\left(x^{\prime}\right)=\omega\left(h_{\sigma}\right)_{i}{ }^{k} \omega\left(h_{\sigma}\right)_{j}{ }^{l}\left(\tau_{1}\right)_{\dot{I}}{ }^{\dot{K}}\left(\tau_{1}\right)_{\dot{J}}{ }^{\dot{L}} B_{k \dot{K} ; l \dot{L}}(x) \\
H_{i \dot{I} ; j \dot{J} ; k \dot{K}}(x)^{\prime}=H_{i \dot{I} ; j \dot{j} ; k \dot{K}}\left(x^{\prime}\right) \\
=\omega\left(h_{\sigma}\right)_{i}{ }^{l} \omega\left(h_{\sigma}\right)_{j}{ }^{m} \omega\left(h_{\sigma}\right)_{k}{ }^{n}\left(\tau_{1}\right)_{\dot{I}} \dot{L}^{\dot{ }}\left(\tau_{1}\right)_{\dot{j}}{ }^{\dot{M}}\left(\tau_{1}\right)_{\dot{K}}{ }^{\dot{N}} H_{l \dot{L} ; m \dot{M} ; n \dot{N}}(x)
\end{gathered}
$$

is a symmetry $S^{\prime}=S$ of the strip form (5.6) of the sigma model. In particular, this requirement includes the following symmetry conditions on the original fields

$$
\begin{gathered}
G_{i j}\left(x \omega^{\dagger}\right)=\omega_{i}^{k} \omega_{j}^{l} G_{k l}(x), \quad B_{i j}\left(x \omega^{\dagger}\right)=\omega_{i}^{k} \omega_{j}^{l} B_{k l}(x) \\
H_{i j k}\left(x \omega^{\dagger}\right)=\omega_{i}^{l} \omega_{j}^{m} \omega_{k}^{n} H_{l m n}(x)
\end{gathered}
$$

which describe the class of nonlinear sigma models with a linear symmetry [24. Linearity of the symmetry conditions can be maintained only in certain preferred coordinate systems: 
In conventional terms, we limit ourselves to the special case of a symmetry $h_{\sigma}$ with one fixed point of $M$, and the preferred coordinate system is that in which the fixed point is at the origin. In a somewhat more general language

$$
h_{\sigma} \in A u t(\text { Iso } M)
$$

and $h_{\sigma}$ must also preserve the 2 -form $B$ on $M$.

When $M$ is a group manifold and $h_{\sigma} \in \operatorname{Aut}(g)$, we already know (see Eq. (4.16)) that the linear symmetry conditions (5.10) are satisfied for the WZW model on $g$, and hence for any principal chiral model on $g$. In the case of the coset CFTs, the symmetry conditions (5.10) will also be satisfied so long as the $g / h$ coset construction [6, [7, 33] is $h_{\sigma}$-invariant: This means in particular that the subalgebra $h \subset g$ is an $h_{\sigma}$-covariant subalgebra of $g$, as discussed for the $H$-invariant coset constructions in Refs. 4, [5, 23.

When the space-time parities (5.4) and the linear symmetry conditions (5.10) are satisfied for all

$$
\hat{h}_{\sigma}=\tau_{1} \times h_{\sigma} \in H_{-} \subset A u t(\text { Iso } M)
$$

we will say that the sigma model is $H_{-}$-symmetric and denote it by $A_{M}\left(H_{-}\right)$. The discussion below is limited to the sigma models in this class.

\subsection{The Fields of the Sigma-Model Orientation Orbifolds}

The sigma-model eigenfields $\mathcal{G}, \mathcal{B}, \mathcal{H}$ are constructed as shown in Eqs. (4.17h-j), but now with the WZW eigenvector matrix $U(\sigma)$ replaced by the eigenvector matrix of the Einsteinspace H-eigenvalue problem [24]

$$
\omega\left(h_{\sigma}\right)_{i}{ }^{j} U^{\dagger}(\sigma)_{j}{ }^{n(r) \mu}=U^{\dagger}(\sigma)_{i}{ }^{n(r) \mu} E_{n(r)}(\sigma), \quad U^{\dagger}(\sigma) U(\sigma)=\mathbb{1}, \quad E_{n(r)}(\sigma)=e^{-2 \pi i \frac{n(r)}{\rho(\sigma)}}
$$

where $\omega\left(h_{\sigma}\right)$ appears in the general orientation-reversing automorphism (5.9).

To go to the open-string sectors of the sigma-model orientation orbifold $A_{M}\left(H_{-}\right) / H_{-}$, we then apply the principle of local isomorphisms

$$
x \underset{\sigma}{\longrightarrow} \hat{x}, \quad \mathcal{G} \underset{\sigma}{\longrightarrow} \hat{G}, \quad \mathcal{B} \underset{\sigma}{\longrightarrow} \hat{B}, \quad \mathcal{H} \underset{\sigma}{\longrightarrow} \hat{H}
$$

as above. For the twisted fields of these open-string sectors, we find the two-component 
structure

$$
\begin{gathered}
\hat{x}_{\sigma}^{n(r) \mu u} \equiv \hat{x}_{\sigma}^{n(r) \mu u}(\xi, t), \quad \hat{\partial}_{n(r) \mu u}(\xi)=\frac{\partial}{\partial \hat{x}^{n(r) \mu u}(\xi)}, \quad \bar{u}=0,1 \\
\hat{G}_{n(r) \mu u ; n(s) \nu v}(\hat{x})=\hat{G}_{n(r) \mu ; n(s) \nu}^{(u+v)}(\hat{x}), \quad \hat{B}_{n(r) \mu u ; n(s) \nu v}(\hat{x})=\hat{B}_{n(r) \mu ; n(s) \nu}^{(u+v)}(\hat{x}) \\
\hat{H}_{n(r) \mu u ; n(s) \nu v ; n(t) \delta w}(\hat{x}) \\
=\hat{\partial}_{n(r) \mu u} \hat{B}_{n(s) \nu v ; n(t) \delta w}(\hat{x})+\hat{\partial}_{n(s) \nu v} \hat{B}_{n(t) \delta w ; n(r) \mu u}(\hat{x})+\hat{\partial}_{n(t) \delta w} \hat{B}_{n(r) \mu u ; n(s) \nu v}(\hat{x}) \\
=\hat{H}_{n(r) \mu ; n(s) \nu ; n(t) \delta}^{(u+v+w)}(\hat{x})
\end{gathered}
$$

and the world-sheet parities

$$
\begin{gathered}
\hat{x}_{\sigma}^{n(r) \mu u}(-\xi)=-\hat{x}_{\sigma}^{n(r) \mu v}(\xi)\left(\tau_{3}\right)_{v}{ }^{u}=(-1)^{u+1} \hat{x}_{\sigma}^{n(r) \mu u}(\xi) \\
\hat{G}_{n(r) \mu ; n(s) \nu}^{(w)}(\hat{x}(-\xi))=(-1)^{w} \hat{G}_{n(r) \mu ; n(s) \nu}^{(w)}(\hat{x}(\xi)) \\
\hat{B}_{n(r) \mu ; n(s) \nu}^{(w)}(\hat{x}(-\xi))=(-1)^{w+1} \hat{B}_{n(r) \mu ; n(s) \nu}^{(w)}(\hat{x}(\xi)) \\
\hat{H}_{n(r) \mu ; n(s) \nu ; n(t) \delta}^{(w)}(\hat{x}(-\xi))=(-1)^{w} \hat{H}_{n(r) \mu ; n(s) \nu ; n(t) \delta}^{(w)}(\hat{x}(\xi)) .
\end{gathered}
$$

Both of these results follow by local isomorphisms from the corresponding properties of the eigenfields.

To discuss the twisted fields in further detail, we again recall that the commuting diagrams [3, 24] of orbifold theory hold as well for the orientation orbifolds. We mention in particular the commuting diagram shown in Fig. 3 for the Einstein coordinates of any orbifold.

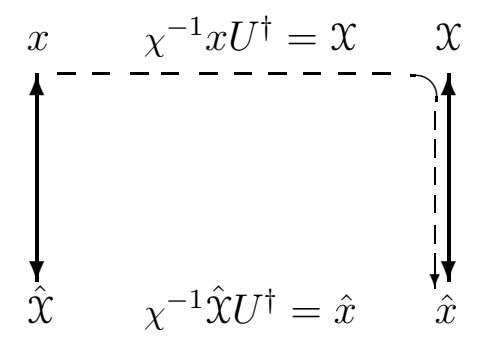

Each vertical double arrow is a local isomorphism

$x=$ coordinates: mixed under automorphisms

$X=$ eigencoordinates: diagonal under automorphisms

$\hat{x}=$ twisted coordinates

$\hat{X}=$ coordinates with twisted boundary conditions

Fig. 3. Coordinates and orbifold coordinates

The fields $\hat{x}$ in Fig. 3 , which are locally isomorphic to the original untwisted Einstein 
coordinates

$$
\begin{gathered}
x^{i \dot{I}}(\xi) \underset{\sigma}{\longrightarrow} \hat{x}_{\sigma}^{i \dot{I}}(\hat{x}(\xi)), \quad \dot{I}=0,1 \\
\hat{x}_{\sigma}^{i \dot{I}}(\hat{x}) \equiv \hat{x}_{\sigma}^{n(r) \mu u} \chi(\sigma)_{n(r) \mu} U(\sigma)_{n(r) \mu}{ }^{i}\left(\sqrt{2} U_{u}^{\dot{I}}\right) \\
\hat{x}_{\sigma}^{n(r) \mu u}(\hat{X})=\hat{x}_{\sigma}^{i \dot{I}} \chi(\sigma)_{n(r) \mu}^{-1} U^{\dagger}(\sigma)_{i}{ }^{n(r) \mu}\left(\frac{1}{\sqrt{2}} U^{\dagger}{ }_{\dot{I}}^{u}\right) \\
\frac{\partial}{\partial \hat{x}^{i \dot{I}}}=\chi(\sigma)_{n(r) \mu}^{-1} U^{\dagger}(\sigma)_{i}{ }^{n(r) \mu}\left(\frac{1}{\sqrt{2}} U^{\dagger} \dot{I}^{u}\right) \hat{\partial}_{n(r) \mu u} \\
\hat{\partial}_{n(r) \mu u}=\chi(\sigma)_{n(r) \mu} U(\sigma)_{n(r) \mu}{ }^{i}\left(\sqrt{2} U_{u}{ }^{\dot{I}}\right) \frac{\partial}{\partial \hat{x}^{i \dot{I}}} \\
\hat{x}_{\sigma}^{i \dot{I}}(\hat{x}(-\xi))=-\hat{x}_{\sigma}^{i \dot{J}}(\hat{x}(\xi))\left(\tau_{1}\right)_{\dot{J}}^{\dot{I}}, \quad \hat{x}^{i \dot{I}}(-\hat{x}(\xi))=-\hat{x}^{i \dot{I}}(\hat{x}(\xi))
\end{gathered}
$$

are generalizations of the Einstein coordinates with twisted boundary conditions [24] in space-time orbifold theory.

Using the diagram in Fig. 3] and following the construction of Ref. 24], we then obtain explicit formulas for the twisted fields of the open-string orientation-orbifold sectors

$$
\begin{aligned}
& \hat{G}_{n(r) \mu u ; n(s) \nu v}(\hat{x})=\chi(\sigma)_{n(r) \mu} \chi(\sigma)_{n(s) \nu} U(\sigma)_{n(r) \mu}{ }^{i} U(\sigma)_{n(s) \nu}{ }^{j} \\
& \times\left(\sqrt{2} U_{u}^{\dot{I}}\right)\left(\sqrt{2} U_{v}^{\dot{j}}\right) G_{i \dot{I} ; j \dot{j}}(\underset{\sigma}{x \rightarrow} \hat{X}(\hat{x})) \\
& \hat{B}_{n(r) \mu u ; n(s) \nu v}(\hat{x}) \equiv \chi(\sigma)_{n(r) \mu} \chi(\sigma)_{n(s) \nu} U(\sigma)_{n(r) \mu}{ }^{i} U(\sigma)_{n(s) \nu}{ }^{j} \\
& \times\left(\sqrt{2} U_{u}^{\dot{I}}\right)\left(\sqrt{2} U_{v}^{\dot{J}}\right) B_{i \dot{I} ; j \dot{j}}(\underset{\sigma}{\rightarrow} \hat{X}(\hat{x})) \\
& \hat{H}_{n(r) \mu u ; n(s) \nu v ; n(t) \delta w}(\hat{x}) \equiv \chi(\sigma)_{n(r) \mu} \chi(\sigma)_{n(s) \nu} \chi(\sigma)_{n(t) \delta} U(\sigma)_{n(r) \mu}{ }^{i} U(\sigma)_{n(s) \nu}{ }^{j} U(\sigma)_{n(t) \delta}{ }^{k} \\
& \times\left(\sqrt{2} U_{u}^{\dot{I}}\right)\left(\sqrt{2} U_{v}^{\dot{J}}\right)\left(\sqrt{2} U_{w}^{\dot{K}}\right) H_{i \dot{I} ; j \dot{j} ; k \dot{K}}(\underset{\sigma}{\rightarrow} \hat{X}(\hat{x}))
\end{aligned}
$$

in terms of the untwisted fields $G, B$, and $H$ of the symmetric sigma model. If we insert the explicit untwisted forms of $G, B$ and $H$ given in Eqs. $(4.7 \mathrm{c}, \mathrm{d})$ for the special case of WZW, the general results in Eq. (5.18) reduce to the explicit formulas $(4.29 \mathrm{c}, \mathrm{d})$ given above for the WZW orientation orbifolds.

In the general case, the explicit formulae (5.18) can be further evaluated as

$$
\hat{x}_{\sigma}^{i \dot{I}}(\hat{x})=\left(\hat{x}_{\sigma}^{n(r) \mu 0}+(-1)^{\dot{I}} \hat{x}_{\sigma}^{n(r) \mu 1}\right) \chi(\sigma)_{n(r) \mu} U(\sigma)_{n(r) \mu}{ }^{i}
$$




$$
\begin{aligned}
& \hat{G}_{n(r) \mu u ; n(s) \nu v}(\hat{x})=\hat{G}_{n(r) \mu ; n(s) \nu}^{(u+v)}(\hat{x}) \\
& \hat{G}_{n(r) \mu ; n(s) \nu}^{(w)}(\hat{x}) \\
& =\chi_{n(r) \mu} \chi_{n(s) \nu} U_{n(r) \mu}{ }^{i} U_{n(s) \nu}{ }^{j}\left(G_{i j}\left(x_{\sigma}^{0} \underset{\sigma}{\rightarrow} \hat{X}_{\sigma}^{0}(\hat{x})\right)+(-1)^{w} G_{i j}\left(x_{\sigma}^{1} \underset{\sigma}{\rightarrow} \hat{X}_{\sigma}^{1}(\hat{x})\right)\right) \\
& \hat{B}_{n(r) \mu u ; n(s) \nu v}(\hat{x})=\hat{B}_{n(r) \mu ; n(s) \nu}^{(u+v)}(\hat{x}) \\
& \hat{B}_{n(r) \mu ; n(s) \nu}^{(w)}(\hat{x}) \\
& =\chi_{n(r) \mu} \chi_{n(s) \nu} U_{n(r) \mu}{ }^{i} U_{n(s) \nu}{ }^{j}\left(B_{i j}\left(x_{\sigma}^{0} \underset{\sigma}{\rightarrow} \hat{x}_{\sigma}^{0}(\hat{x})\right)+(-1)^{w} B_{i j}\left(x^{1} \underset{\sigma}{\rightarrow} \hat{X}_{\sigma}^{1}(\hat{x})\right)\right) \\
& \hat{H}_{n(r) \mu u ; n(s) \nu v ; n(t) \delta w}(\hat{x})=\hat{H}_{n(r) \mu ; n(s) \nu}^{(u+v+w)}(\hat{x}) \\
& \hat{H}_{n(r) \mu ; n(s) \nu ; n(t) \delta}^{(w)}(\hat{x})=\chi_{n(r) \mu} \chi_{n(s) \nu} \chi_{n(t) \delta} U_{n(r) \mu}{ }^{i} U_{n(s) \nu}{ }^{j} U_{n(t) \delta}^{k} \\
& \times\left(H_{i j k}\left(x_{\sigma}^{0} \underset{\sigma}{\rightarrow} \hat{X}_{\sigma}^{0}(\hat{x})\right)+(-1)^{w} H_{i j k}\left(x_{\sigma}^{1} \underset{\sigma}{\rightarrow} \hat{X}_{\sigma}^{1}(\hat{x})\right)\right)
\end{aligned}
$$

where we have suppressed some $\sigma$-dependence. These results are easily obtained from the forms in Eq. (5.18) by doing the sums on the internal two-dimensional indices.

\subsection{The Sigma-Model Orientation-Orbifold Action}

The principle of local isomorphisms also gives us the sigma-model orientation-orbifold action

$$
\begin{gathered}
S \underset{\sigma}{\longrightarrow} \hat{S}_{\sigma} \\
\hat{S}_{\sigma}=\int d t \int_{0}^{\pi} d \xi \hat{\mathcal{L}}_{\sigma} \\
\hat{\mathcal{L}}_{\sigma}=\frac{1}{8 \pi}\left(\hat{G}_{n(r) \mu u ; n(s) \nu v}(\hat{x})+\hat{B}_{n(r) \mu u ; n(s) \nu v}(\hat{x})\right) \partial_{+} \hat{x}_{\sigma}^{n(r) \mu u} \partial_{-} \hat{x}_{\sigma}^{n(s) \nu v} \\
=\frac{1}{8 \pi} \sum_{w=0}^{1}\left(\hat{G}_{n(r) \mu ; n(s) \nu}^{(w)}(\hat{x})+\hat{B}_{n(r) \mu ; n(s) \nu}^{(w)}(\hat{x})\right) \sum_{u=0}^{1} \partial_{+} \hat{x}_{\sigma}^{n(r) \mu u} \partial_{-} \hat{x}_{\sigma}^{n(s) \nu, w-u} \\
\hat{\mathcal{L}}_{\sigma}(-\xi)=\hat{\mathcal{L}}_{\sigma}(\xi)
\end{gathered}
$$

by the standard derived isomorphism from the eigenfield form of $S$. This action, which describes all open-string sectors

$$
\hat{h}_{\sigma}=\tau_{1} \times h_{\sigma} \in H_{-}, \quad H_{-} \subset \operatorname{Aut}(\text { Iso } M)
$$


of the sigma-model orientation orbifold $A_{M}\left(H_{-}\right) / H_{-}$, is another central result of this paper.

For this action, the following variational boundary conditions which describe the sigmamodel orientation-orbifold branes

$$
\begin{aligned}
\delta \hat{x}^{n(r) \mu u}(\xi)\left(\hat{G}_{n(r) \mu u ; n(s) \nu v}(\hat{x}(\xi)) \partial_{\xi}-\hat{B}_{n(r) \mu u ; n(s) \nu v}(\hat{x}(\xi)) \partial_{t}\right) \hat{x}^{n(s) \nu v}(\xi) \\
=\sum_{u, v=0}^{1} \delta \hat{x}^{n(r) \mu u}(\xi)\left(\hat{G}_{n(r) \mu ; n(s) \nu}^{(u+v)}(\hat{x}(\xi)) \partial_{\xi}-\hat{B}_{n(r) \mu ; n(s) \nu}^{(u+v)}(\hat{x}(\xi)) \partial_{t}\right) \hat{x}^{n(s) \nu v}(\xi) \\
=0 \text { at } \xi=0, \pi
\end{aligned}
$$

are obtained in the standard manner by requiring that the bulk and boundary terms cancel separately in a general variation of the fields. The more explicit form of these boundary conditions

$$
\begin{aligned}
& {\left[\delta \hat{x}^{n(r) \mu 0}(\xi)\left(\hat{G}_{n(r) \mu ; n(s) \nu}^{(0)}(\hat{x}(\xi)) \partial_{\xi}-\hat{B}_{n(r) \mu ; n(s) \nu}^{(0)}(\hat{x}(\xi)) \partial_{t}\right) \hat{x}^{n(s) \nu 0}(\xi)+\right.} \\
& \delta \hat{x}^{n(r) \mu 0}(\xi)\left(\hat{G}_{n(r) \mu ; n(s) \nu}^{(1)}(\hat{x}(\xi)) \partial_{\xi}-\hat{B}_{n(r) \mu ; n(s) \nu}^{(1)}(\hat{x}(\xi)) \partial_{t}\right) \hat{x}^{n(s) \nu 1}(\xi)+ \\
& \delta \hat{x}^{n(r) \mu 1}(\xi)\left(\hat{G}_{n(r) \mu ; n(s) \nu}^{(1)}(\hat{x}(\xi)) \partial_{\xi}-\hat{B}_{n(r) \mu ; n(s) \nu}^{(1)}(\hat{x}(\xi)) \partial_{t}\right) \hat{x}^{n(s) \nu 0}(\xi)+ \\
& \left.\delta \hat{x}^{n(r) \mu 1}(\xi)\left(\hat{G}_{n(r) \mu ; n(s) \nu}^{(0)}(\hat{x}(\xi)) \partial_{\xi}-\hat{B}_{n(r) \mu ; n(s) \nu}^{(0)}(\hat{x}(\xi)) \partial_{t}\right) \hat{x}^{n(s) \nu 1}(\xi)\right]=0 \text { at } \xi=0, \pi
\end{aligned}
$$

will be useful in the following subsection.

The classical bulk equations of motion for the action (5.20) are given in Sec. 6, where we develop the necessary twisted Christoffel symbols.

We finally mention the untwisted two-component interval $d s^{2}$ and the orientation orbifold interval $d \hat{s}^{2}$

$$
\begin{aligned}
d s^{2}(\xi) & =G_{i \dot{I} ; j}(x(\xi)) d x^{i \dot{I}}(\xi) d x^{j \dot{J}}(\xi) \\
& =G_{i j}(x(\xi)) d x^{i}(\xi) d x^{j}(\xi)+(\xi \rightarrow-\xi) \\
d \hat{s}^{2}(\xi) & =\hat{G}_{n(r) \mu u ; n(s) \nu v}(\hat{x}(\xi)) d \hat{x}^{n(r) \mu u}(\xi) d \hat{x}^{n(s) \nu v}(\xi) \\
& =\sum_{w=0}^{1} \hat{G}_{n(r) \mu ; n(s) \nu}^{(w)}(\hat{x}(\xi)) \sum_{u=0}^{1} d \hat{x}^{n(r) \mu u}(\xi) d \hat{x}^{n(s) \nu, w-u}(\xi)
\end{aligned}
$$

where the space-time parity (5.4) was used to obtain (5.24a). Both of these intervals are even under world-sheet parity $\xi \leftrightarrow-\xi$. 


\subsection{Monodromies and Extra Boundary Conditions}

In this subsection, we consider further structure of the sigma-model orientation-orbifold branes, concentrating primarily but not exclusively on the case $h_{\sigma}^{2}=1$.

For this discussion, we will need the $h_{\sigma}^{2}=1$ monodromies

$$
\begin{gathered}
h_{\sigma}^{2}=1: \quad \text { automorphic responses } \underset{\sigma}{\longrightarrow} \text { monodromies } \\
\hat{x}_{\sigma}^{n(r) \mu u}(\xi+2 \pi)=\hat{x}_{\sigma}^{n(r) \mu u}(\xi) e^{2 \pi i\left(\frac{n(r)}{\rho(\sigma)}+\frac{u}{2}\right)}, \quad \bar{u}=0,1 \\
\hat{G}_{n(r) \mu ; n(s) \nu}^{(w)}(\hat{x}(\xi+2 \pi))=e^{-2 \pi i\left(\frac{n(r)+n(s)}{\rho(\sigma)}+\frac{w}{2}\right)} \hat{G}_{n(r) \mu ; n(s) \nu}^{(w)}(\hat{x}(\xi)) \\
\hat{B}_{n(r) \mu ; n(s) \nu}^{(w)}(\hat{x}(\xi+2 \pi))=e^{-2 \pi i\left(\frac{n(r)+n(s)}{\rho(\sigma)}+\frac{w}{2}\right)} \hat{B}_{n(r) \mu ; n(s) \nu}^{(w)}(\hat{x}(\xi)) \\
\hat{H}_{n(r) \mu ; n(s) \nu ; n(t) \delta}^{(w)}(\hat{x}(\xi+2 \pi))=e^{-2 \pi i\left(\frac{n(r)+n(s)+n(t)}{\rho(\sigma)}+\frac{w}{2}\right)} \hat{H}_{n(r) \mu ; n(s) \nu ; n(t) \delta}^{(w)}(\hat{x}(\xi)) \\
\hat{\mathcal{L}}_{\sigma}(\xi+2 \pi)=\hat{\mathcal{L}}_{\sigma}(\xi)
\end{gathered}
$$

which follow in this case by local isomorphisms from the automorphic responses of the eigenfields. These monodromies have the same forms as those found for WZW in Eqs. (4.35) and (4.28). We note in particular that the coordinates $\hat{x}$ with twisted boundary conditions in Eq. (5.17) have mixed monodromy

$$
\hat{x}_{\sigma}^{i \dot{I}}(\hat{x}(\xi+2 \pi))=\hat{x}_{\sigma}^{j \dot{J}}(\hat{x}(\xi)) \omega^{\dagger}\left(h_{\sigma}\right)_{j}{ }^{i}\left(\tau_{1}\right)_{j}^{\dot{I}}
$$

and our coordinates $\hat{x}$ with definite monodromy in Eq. (5.25) are the monodromy decomposition of $\hat{x}$.

Using the world-sheet parities (5.16) and the monodromies (5.25), one finds the brane substructure

$$
\begin{gathered}
\hat{x}_{\sigma}^{n(r) \mu 0}(0)=0, \quad \hat{x}_{\sigma}^{n(r) \mu u}(\pi)=0 \text { unless } \frac{n(r)}{\rho(\sigma)} \in \mathbb{Z}+\frac{1}{2} \\
\left(\partial_{t} \hat{x}_{\sigma}^{n(r) \mu 0}\right)(0)=0, \quad\left(\partial_{t} \hat{x}_{\sigma}^{n(r) \mu u}\right)(\pi)=0 \text { unless } \frac{n(r)}{\rho(\sigma)} \in \mathbb{Z}+\frac{1}{2} \\
\left(\partial_{\xi} \hat{x}_{\sigma}^{n(r) \mu 1}\right)(0)=0, \quad\left(\partial_{\xi} \hat{x}_{\sigma}^{n(r) \mu u}\right)(\pi)=0 \text { unless } \frac{n(r)}{\rho(\sigma)} \in \mathbb{Z} \\
\hat{G}_{n(r) \mu ; n(s) \nu}^{(1)}(\hat{x}(0))=0, \quad \hat{G}_{n(r) \mu ; n(s) \nu}^{(w)}(\hat{x}(\pi))=0 \text { unless } \frac{n(r)+n(s)}{\rho(\sigma)} \in \mathbb{Z} \\
\left(\partial_{t} \hat{G}_{n(r) \mu ; n(s) \nu}^{(1)}\right)(\hat{x}(0))=0, \quad\left(\partial_{t} \hat{G}_{n(r) \mu ; n(s) \nu}^{(w)}\right)(\hat{x}(\pi))=0 \text { unless } \frac{n(r)+n(s)}{\rho(\sigma)} \in \mathbb{Z} \\
\left(\partial_{\xi} \hat{G}_{n(r) \mu ; n(s) \nu}^{(0)}\right)(\hat{x}(0))=0, \quad\left(\partial_{\xi} \hat{G}_{n(r) \mu ; n(s) \nu}^{(w)}\right)(\hat{x}(\pi))=0 \text { unless } \frac{n(r)+n(s)}{\rho(\sigma)} \in \mathbb{Z}+\frac{1}{2}
\end{gathered}
$$




$$
\begin{gathered}
\hat{B}_{n(r) \mu ; n(s) \nu}^{(0)}(\hat{x}(0))=0, \quad \hat{B}_{n(r) \mu ; n(s) \nu}^{(w)}(\hat{x}(\pi))=0 \text { unless } \frac{n(r)+n(s)}{\rho(\sigma)} \in \mathbb{Z}+\frac{1}{2} \\
\left(\partial_{t} \hat{B}_{n(r) \mu ; n(s) \nu}^{(0)}\right)(\hat{x}(0))=0, \quad\left(\partial_{t} \hat{B}_{n(r) \mu ; n(s) \nu}^{(w)}\right)(\hat{x}(\pi))=0 \text { unless } \frac{n(r)+n(s)}{\rho(\sigma)} \in \mathbb{Z}+\frac{1}{2} \\
\left(\partial_{\xi} \hat{B}_{n(r) \mu ; n(s) \nu}^{(1)}\right)(\hat{x}(0))=0, \quad\left(\partial_{\xi} \hat{B}_{n(r) \mu ; n(s) \nu}^{(w)}\right)(\hat{x}(\pi))=0 \text { unless } \frac{n(r)+n(s)}{\rho(\sigma)} \in \mathbb{Z} \\
\hat{H}_{n(r) \mu ; n(s) \nu ; n(t) \delta}^{(1)}(\hat{x}(0))=0, \quad \hat{H}_{n(r) \mu ; n(s) \nu ; n(t) \delta}^{(w)}(\hat{x}(\pi))=0 \text { unless } \frac{n(r)+n(s)+n(t)}{\rho(\sigma)} \in \mathbb{Z} \\
\left(\partial_{t} \hat{H}_{n(r) \mu ; n(s) \nu ; n(t) \delta}^{(1)}\right)(\hat{x}(0))=0 \\
\left(\partial_{t} \hat{H}_{n(r) \mu ; n(s) \nu ; n(t) \delta}^{(w)}\right)(\hat{x}(\pi))=0 \text { unless } \frac{n(r)+n(s)+n(t)}{\rho(\sigma)} \in \mathbb{Z} \\
\left(\partial_{\xi} \hat{H}_{n(r) \mu ; n(s) \nu ; n(t) \delta}^{(0)}\right)(\hat{x}(0))=0 \\
\left(\partial_{\xi} \hat{H}_{n(r) \mu ; n(s) \nu ; n(t) \delta}^{(w)}\right)(\hat{x}(\pi))=0 \text { unless } \frac{n(r)+n(s)+n(t)}{\rho(\sigma)} \in \mathbb{Z}+\frac{1}{2}
\end{gathered}
$$

which has the same form as that found in Subsec. 4.6 for the special case of the WZW orientation orbifolds. Note in particular that the coordinates of the basic sector with $h_{\sigma}=1$ are Dirichlet-Dirichlet and Neumann-Dirichlet for $\hat{x}_{\sigma}^{0 \mu 0}$ and $\hat{x}_{\sigma}^{0 \mu 1}$ respectively, while the generic sector with $h_{\sigma}^{2}=1, h_{\sigma} \neq 1$ contains all four coordinate types, D-D, N-D, D-N and $\mathrm{N}-\mathrm{N}{ }^{\ddagger 7}$.

We turn next to explicitly verify the variational boundary conditions (15.22), (15.23) given in the previous subsection, using the $\hat{x}, \hat{G}$ and $\hat{B}$ boundary conditions above. In particular, the boundary conditions $(5.27 \mathrm{a}-\mathrm{c})$ on $\hat{x}$ allow us to reduce the variational boundary condition (5.23) at $\xi=0$ to the form

$$
\begin{aligned}
& \sum_{u, v=0}^{1} \delta \hat{x}^{n(r) \mu u}(\xi)\left(\hat{G}_{n(r) \mu ; n(s) \nu}^{(u+v)}(\hat{x}(\xi)) \partial_{\xi}-\hat{B}_{n(r) \mu ; n(s) \nu}^{(u+v)}(\hat{x}(\xi)) \partial_{t}\right) \hat{x}^{n(s) \nu v}(\xi)= \\
& \delta \hat{x}^{n(r) \mu 1}(\xi)\left(\hat{G}_{n(r) \mu ; n(s) \nu}^{(1)}(\hat{x}(\xi)) \partial_{\xi} \hat{x}^{n(s) \nu 0}(\xi)-\hat{B}_{n(r) \mu ; n(s) \nu}^{(0)}(\hat{x}(\xi)) \partial_{t} \hat{x}^{n(s) \nu 1}(\xi)\right) \text { at } \xi=0 .
\end{aligned}
$$

and this quantity vanishes by the boundary conditions of $\hat{G}$ and $\hat{B}$ given in Eqs. (5.27d) and (5.27g).

To study $\xi=\pi$, we consider first the generic $h_{\sigma}^{2}=1$ sector with $\rho(\sigma)=2$ in Eq. (2.30b). In this case, we instead expand the sum (5.22) in terms of the spectral indices $\bar{n}(r), \bar{n}(s) \in$

\footnotetext{
${ }^{\ddagger}$ Half-integer moded scalar fields [8] and the corresponding twisted open strings with D-N or N-D boundary conditions [34, 35] provided the first examples of twisted sectors of orbifolds.
} 
$\{0,1\}:$

$$
\begin{array}{r}
\sum_{u, v=0}^{1}\left[\delta \hat{x}^{0 \mu u}(\xi)\left(\hat{G}_{0 \mu ; 0 \nu}^{(u+v)}(\hat{x}(\xi)) \partial_{\xi}-\hat{B}_{0 \mu ; 0 \nu}^{(u+v)}(\hat{x}(\xi)) \partial_{t}\right) \hat{x}^{0 \nu v}(\xi)+\right. \\
\delta \hat{x}^{0 \mu u}(\xi)\left(\hat{G}_{0 \mu ; 1 \nu}^{(u+v)}(\hat{x}(\xi)) \partial_{\xi}-\hat{B}_{0 \mu ; 1 \nu}^{(u+v)}(\hat{x}(\xi)) \partial_{t}\right) \hat{x}^{1 \nu v}(\xi)+ \\
\quad \hat{x}^{1 \mu u}(\xi)\left(\hat{G}_{1 \mu ; 0 \nu}^{(u+v)}(\hat{x}(\xi)) \partial_{\xi}-\hat{B}_{1 \mu ; 0 \nu}^{(u+v)}(\hat{x}(\xi)) \partial_{t}\right) \hat{x}^{0 \nu v}(\xi)+ \\
\left.\delta \hat{x}^{1 \mu u}(\xi)\left(\hat{G}_{1 \mu ; 1 \nu}^{(u+v)}(\hat{x}(\xi)) \partial_{\xi}-\hat{B}_{1 \mu ; 1 \nu}^{(u+v)}(\hat{x}(\xi)) \partial_{t}\right) \hat{x}^{1 \nu v}(\xi)\right] .
\end{array}
$$

With this expansion and the explicit form of the boundary conditions

$$
\begin{gathered}
\hat{x}^{0 \mu u}(\pi)=\delta \hat{x}^{0 \mu u}(\pi)=\partial_{t} \hat{x}^{0 \mu u}(\pi)=0 \\
\partial_{\xi} \hat{x}^{1 \mu u}(\pi)=\hat{G}_{1 \mu ; 0 \nu}^{(u+v)}(\hat{x}(\pi))=\hat{B}_{1 \mu ; 1 \nu}^{(u+v)}(\hat{x}(\pi))=0
\end{gathered}
$$

it is straightforward to check that this quantity vanishes at $\xi=\pi$. For the basic open-string sector (2.30a) with $h_{\sigma}=\rho(\sigma)=1$, the same conclusion is reached

$$
\begin{aligned}
& \hat{x}^{0 \mu u}(\pi)=\delta \hat{x}^{0 \mu u}(\pi)=\partial_{t} \hat{x}^{0 \mu u}(\pi)=0 \\
& \quad \Rightarrow \delta \hat{x}^{0 \mu u}(\pi)\left(\hat{G}_{0 \mu ; 0 \nu}^{(u+v)}(\hat{x}(\pi)) \partial_{\xi}-\hat{B}_{0 \mu ; 0 \nu}^{(u+v)}(\hat{x}(\pi)) \partial_{t}\right) \hat{x}^{0 \nu v}(\pi)=0
\end{aligned}
$$

because only the $\bar{n}(r)=0$ terms survive in this case.

We finally emphasize that, as usual, all the boundary conditions at $\xi=0$ in this subsection follow directly from world-sheet parity, and hence hold for all $\hat{h}_{\sigma}$.

\subsection{Example: The Coset Orientation-Orbifold Action}

We give here a brief sketch of our results for coset orientation orbifolds, following the discussion of ordinary coset orbifolds in Refs. 4, 5, 23, 24.

To build the coset orientation orbifolds $A_{g / h}\left(H_{-}\right) / H_{-}$at the operator level, one begins with a left- and right-mover copy of any $g / h$ coset construction [6, 7, 33, 12]

$$
\begin{gathered}
J_{h} \subset J_{g}, \quad T_{g / h}=T_{g}-T_{h} \\
\bar{J}_{h} \subset \bar{J}_{g}, \quad \bar{T}_{g / h}=\bar{T}_{g}-\bar{T}_{h} \\
c_{g / h}=\bar{c}_{g / h}=c_{g}-c_{h}
\end{gathered}
$$


where $T_{g}$ and $T_{h}$ are the affine-Sugawara constructions $[6,7,36-38,12]$ of $g \supset h$. For our construction, we will assume that $G / H$ is a reductive coset space and that the coset construction is $h_{\sigma}$-invariant:

$$
\frac{g}{h}=\frac{g}{h}\left(h_{\sigma}\right), \quad h_{\sigma} \in \operatorname{Aut}(g), \quad h_{\sigma} \in A u t(h) .
$$

As studied in the orbifold program under the rubric of $H$-invariant coset constructions 44, 5. 23, 24, $h_{\sigma}$-invariance of the coset construction means that $h_{\sigma}$ is an automorphism of $g$, and $h \subset g$ transforms covariantly under $h_{\sigma}$. Each such system is then automatically invariant under the general orientation-reversing automorphism $\hat{h}_{\sigma}=\tau_{1} \times h_{\sigma}$, in agreement with the results of App. B. Then the method of eigenfields and the principle of local isomorphisms gives the twisted currents and stress tensors of sector $\hat{h}_{\sigma}$

$$
\hat{J}_{\hat{\mathfrak{h}}_{O}(\sigma)} \subset \hat{J}_{\hat{\mathfrak{g}}_{O}(\sigma)}, \quad \hat{T}_{\hat{\mathfrak{g}}_{O}(\sigma) / \hat{\mathfrak{h}}_{O}(\sigma)}=\hat{T}_{\hat{\mathfrak{g}}_{O}(\sigma)}-\hat{T}_{\hat{\mathfrak{h}}_{O}(\sigma)}, \quad \hat{c}_{\hat{\mathfrak{g}}_{O}(\sigma) / \hat{\mathfrak{h}}_{O}(\sigma)}=2 c_{g / h}
$$

as well as the half-integrally moded Virasoro generators of Refs. [1, 25]. The individual stress tensors $\hat{T}_{\hat{\mathfrak{g}}_{O}(\sigma)}$ and $\hat{T}_{\hat{\mathfrak{h}}_{O}(\sigma)}$ are the twisted affine-Sugawara constructions [25] associated to the twisted current algebras $\hat{\mathfrak{g}}_{O}(\sigma) \supset \hat{\mathfrak{h}}_{O}(\sigma)$ of the corresponding WZW orientation orbifolds.

At the classical level, we begin with the general gauged WZW model [39-43,23]

$$
\begin{gathered}
S_{g / h}\left[M, g, A_{ \pm} ; \Gamma\right]=S_{W Z W}[M, g ; \Gamma]+\frac{1}{4 \pi} \int d t \int_{0}^{2 \pi} d \xi \operatorname{Tr}\left(M \left(g^{-1} \partial_{+} g\left(i A_{-}\right)\right.\right. \\
\left.\left.-i A_{+} \partial_{-} g g^{-1}-g^{-1} A_{+} g A_{-}+A_{+} A_{-}\right)\right) \\
A_{+}=-i h_{+}^{-1} \partial_{+} h_{+}, \quad A_{-}=-i h_{-} \partial_{-} h_{-}^{-1}
\end{gathered}
$$

for the $g / h$ coset construction, where $M$ is the data matrix in Eq. (2.4). In agreement with the operator argument above, this action is invariant under the general orientation-reversing automorphism $\hat{h}_{\sigma}=\tau_{1} \times h_{\sigma}$

$$
\begin{aligned}
& A_{ \pm}(\xi)^{\prime}=W\left(h_{\sigma} ; T\right) A_{\mp}(-\xi) W^{\dagger}\left(h_{\sigma} ; T\right) \\
& h_{ \pm}(\xi)^{\prime}=W\left(h_{\sigma} ; T\right) h_{\mp}^{-1}(-\xi) W^{\dagger}\left(h_{\sigma} ; T\right) \\
& S_{g / h}\left[M, g^{\prime}, A_{ \pm}^{\prime} ; \Gamma\right]=S_{g / h}\left[M, g, A_{ \pm} ; \Gamma\right]
\end{aligned}
$$

when the $g / h$ coset construction is $h_{\sigma}$-invariant. Here $W$ is the action of $h_{\sigma}$ in rep $T$ and $g^{\prime}$ is given in Eq. (2.8a). 
In this case, the appropriate two-component gauge fields are:

$$
\begin{gathered}
\tilde{A}_{ \pm}(\xi) \equiv\left(\begin{array}{cc}
A_{ \pm}(\xi) & 0 \\
0 & A_{\mp}(-\xi)
\end{array}\right), \quad \tilde{h}_{ \pm}(\xi) \equiv\left(\begin{array}{cc}
h_{ \pm}(\xi) & 0 \\
0 & h_{\mp}^{-1}(-\xi)
\end{array}\right) \\
\tilde{A}_{+}(\xi)=-i \tilde{h}_{+}^{-1} \partial_{+} \tilde{h}_{+}, \quad \tilde{A}_{-}(\xi)=-i \tilde{h}_{-} \partial_{-} \tilde{h}_{-}^{-1} \\
\tilde{A}_{ \pm}(-\xi)=\tau_{1} \tilde{A}_{\mp}(\xi) \tau_{1}, \quad \tilde{h}_{ \pm}(-\xi)=\tau_{1} \tilde{h}_{\mp}(\xi) \tau_{1} \\
\tilde{A}_{ \pm}(\xi)^{\prime}=\tau_{1} W\left(h_{\sigma} ; T\right) \tilde{A}_{ \pm}(\xi) W^{\dagger}\left(h_{\sigma} ; T\right) \tau_{1} \\
\tilde{h}_{ \pm}(\xi)^{\prime}=\tau_{1} W\left(h_{\sigma} ; T\right) \tilde{h}_{ \pm}(\xi) W^{\dagger}\left(h_{\sigma} ; T\right) \tau_{1} .
\end{gathered}
$$

A two-component gauge transformation matrix $\tilde{\psi}(\xi)$ is also necessary

$$
\tilde{\psi}(\xi) \equiv\left(\begin{array}{cc}
\psi(\xi) & 0 \\
0 & \psi(-\xi)
\end{array}\right), \quad \tilde{A}_{ \pm} \rightarrow \tilde{A}_{ \pm}^{\tilde{\psi}}=\tilde{\psi} \tilde{A}_{ \pm} \tilde{\psi}^{-1}+i \partial_{ \pm} \tilde{\psi} \psi^{-1}
$$

to put the standard gauge transformation of $A_{ \pm}$into two-component form. The twocomponent form of the gauged action on $\Gamma_{1 / 2}$ is then easily worked out

$$
\begin{aligned}
& S_{g / h}\left[M \otimes \mathbb{1}_{2}, \tilde{g}, \tilde{A}_{ \pm} ; \Gamma_{\frac{1}{2}}\right] \equiv S_{W Z W}\left[M \otimes \mathbb{1}_{2}, \tilde{g} ; \Gamma_{\frac{1}{2}}\right]+ \\
& +\frac{1}{4 \pi} \int d t \int_{0}^{\pi} d \xi \widehat{\operatorname{Tr}}\left(\left(M \otimes \mathbb{1}_{2}\right)\left(\tilde{g}^{-1} \partial_{+} \tilde{g}\left(i \tilde{A}_{-}\right)-i \tilde{A}_{+} \partial_{-} \tilde{g} \tilde{g}^{-1}-\tilde{g}^{-1} \tilde{A}_{+} \tilde{g} \tilde{A}_{-}+\tilde{A}_{+} \tilde{A}_{-}\right)\right) \\
& \quad=S_{g / h}\left[M, g, A_{ \pm} ; \Gamma\right]
\end{aligned}
$$

where the WZW action on $\Gamma_{1 / 2}$ is given in Eq. (3.4).

The extra eigenfields $\mathcal{A}_{ \pm}, \mathfrak{h}_{ \pm}$and $\Psi$ associated with the gauging

$$
\begin{gathered}
\mathcal{O}(\mathcal{T}, \xi, \sigma) \equiv U U(T, \sigma) \tilde{O}(T, \xi) U^{\dagger}(T, \sigma) U^{\dagger} \\
\mathcal{O}=\mathcal{G}, \mathcal{A}_{ \pm}, \mathfrak{h}_{ \pm}, \Psi, \quad \tilde{O}=\tilde{g}, \tilde{A}_{ \pm}, \tilde{h}_{ \pm}, \tilde{\psi}
\end{gathered}
$$

are constructed in analogy to the eigengroup elements in Eq. (2.16a). The usual structure of these eigenfields is easily worked out, but we omit the details for brevity. Then the principle of local isomorphisms

$$
\mathcal{G} \underset{\sigma}{\longrightarrow} \hat{g}, \quad \mathcal{A}_{ \pm} \underset{\sigma}{\longrightarrow} \hat{A}_{ \pm}, \quad \mathfrak{h}_{ \pm} \underset{\sigma}{\longrightarrow} \hat{h}_{ \pm}, \quad \Psi \underset{\sigma}{\longrightarrow} \hat{\psi}
$$

gives the following results in the open-string sectors of the general coset orientation orbifold. 
In the first place, we record the twisted Polyakov-Weigmann identity [23] on $\Gamma_{1 / 2}$

$$
\begin{aligned}
\hat{S}_{\hat{g}_{O}(\sigma)}\left[\mathcal{M} \otimes \mathbb{1}_{2}, \hat{g} \hat{h} ; \Gamma_{\frac{1}{2}}\right]= & \hat{S}_{\hat{g}_{O}(\sigma)}\left[\mathcal{M} \otimes \mathbb{1}_{2}, \hat{g} ; \Gamma_{\frac{1}{2}}\right]+\hat{S}_{\hat{g}_{O}(\sigma)}\left[\mathcal{M} \otimes \mathbb{1}_{2}, \hat{h} ; \Gamma_{\frac{1}{2}}\right] \\
& -\frac{1}{4 \pi} \int d t \int_{0}^{\pi} d \xi \widehat{\operatorname{Tr}}\left(\left(\mathcal{M} \otimes \mathbb{1}_{2}\right) \hat{g}^{-1} \partial_{+} \hat{g} \partial_{-} \hat{h} \hat{h}^{-1}\right)
\end{aligned}
$$

where the WZW orientation-orbifold action $\hat{S}_{\hat{g}_{O}(\sigma)}$ on $\Gamma_{1 / 2}$ is given in Eq. (3.9)). We also obtain the general coset orientation-orbifold action

$$
\begin{gathered}
\hat{S}_{\hat{g}_{O}(\sigma) / \hat{h}_{O}(\sigma)}\left[\hat{g}, \hat{A}_{ \pm} ; \Gamma_{\frac{1}{2}}\right] \equiv \hat{S}_{\hat{g}_{O}(\sigma)}\left[\mathcal{M} \otimes \mathbb{1}_{2}, \hat{g} ; \Gamma_{\frac{1}{2}}\right] \\
+\frac{1}{4 \pi} \int d t \int_{0}^{\pi} d \xi \widehat{\operatorname{Tr}}\left(\left(\mathcal{M} \otimes \mathbb{1}_{2}\right)\left(\hat{g}^{-1} \partial_{+} \hat{g}\left(i \hat{A}_{-}\right)-i \hat{A}_{+} \partial_{-} \hat{g} \hat{g}^{-1}-\hat{g}^{-1} \hat{A}_{+} \hat{g} \hat{A}_{-}+\hat{A}_{+} \hat{A}_{-}\right)\right) \\
\hat{A}_{+} \equiv-i \hat{h}_{+}^{-1} \partial_{+} \hat{h}_{+}, \quad \hat{A}_{-} \equiv-i \hat{h}_{-} \partial_{-} \hat{h}_{-}^{-1} \\
\hat{g} \in \widehat{\mathfrak{G}}_{O}(\sigma), \quad \hat{h}_{ \pm} \in \widehat{\mathfrak{H}}_{O}(\sigma) \subset \widehat{\mathfrak{G}}_{O}(\sigma), \quad \hat{A}_{ \pm} \in \hat{h}_{O}(\sigma) \subset \hat{g}_{O}(\sigma) \\
{\left[\mathcal{M} \otimes \mathbb{1}_{2}, \hat{g}\right]=\left[\mathcal{M} \otimes \mathbb{1}_{2}, \hat{h}_{ \pm}\right]=\left[\mathcal{M} \otimes \mathbb{1}_{2}, \hat{A}_{ \pm}\right]=0}
\end{gathered}
$$

which describes open-string sector $\hat{h}_{\sigma}$ of the general coset orientation orbifold $A_{g / h}\left(H_{-}\right) / H_{-}$. Here $\widehat{\mathfrak{G}}_{O}(\sigma)$ is the group formed by the set of all group orbifold elements $\hat{g}$ (exponentiated from $\hat{g}_{O}(\sigma)$ ), the quantities $\hat{h}_{ \pm}$are arbitrary subgroup orbifold elements in the subgroup $\widehat{\mathfrak{H}}_{O}(\sigma)$ (exponentiated from $\hat{h}_{O}(\sigma)$ ) and $\hat{A}_{ \pm}$are the orientation-orbifold matrix gauge fields.

The coset orientation-orbifold action (5.43) is a gauging of the WZW orientationorbifold action (3.9) by a general twisted vector gauge group

$$
\begin{gathered}
\hat{g}(\mathcal{T}, \xi, t, \sigma) \rightarrow \hat{g}(\mathcal{T}, \xi, t, \sigma)^{\hat{\psi}} \equiv \hat{\psi}(\mathcal{T}, \xi, t, \sigma) \hat{g}(\mathcal{T}, \xi, t, \sigma) \hat{\psi}^{-1}(\mathcal{T}, \xi, t, \sigma) \\
\hat{h}_{+}(\mathcal{T}, \xi, t, \sigma) \rightarrow \hat{h}_{+}(\mathcal{T}, \xi, t, \sigma)^{\hat{\psi}} \equiv \hat{h}_{+}(\mathcal{T}, \xi, t, \sigma) \hat{\psi}^{-1}(\mathcal{T}, \xi, t, \sigma) \\
\hat{h}_{-}(\mathcal{T}, \xi, t, \sigma) \rightarrow \hat{h}_{-}(\mathcal{T}, \xi, t, \sigma)^{\hat{\psi}} \equiv \hat{\psi}(\mathcal{T}, \xi, t, \sigma) \hat{h}_{-}(\mathcal{T}, \xi, t, \sigma) \\
\hat{A}_{ \pm}(\mathcal{T}, \xi, t, \sigma) \rightarrow \hat{A}_{ \pm}(\mathcal{T}, \xi, t, \sigma)^{\hat{\psi}} \equiv \hat{\psi}(\mathcal{T}, \xi, t, \sigma) \hat{A}_{ \pm}(\mathcal{T}, \xi, t, \sigma) \hat{\psi}^{-1}(\mathcal{T}, \xi, t, \sigma) \\
+i \partial_{ \pm} \hat{\psi}(\mathcal{T}, \xi, t, \sigma) \hat{\psi}^{-1}(\mathcal{T}, \xi, t, \sigma) \\
{\left[\mathcal{M} \otimes \mathbb{1}_{2}, \hat{\psi}(\mathcal{T}, \xi, t, \sigma)\right]=0} \\
\hat{S}_{\hat{g}_{O}(\sigma) / \hat{h}_{O}(\sigma)}\left[\mathcal{M} \otimes \mathbb{1}_{2}, \hat{g}^{\hat{\psi}}, \hat{h}_{ \pm}^{\hat{\psi}} ; \Gamma_{\frac{1}{2}}\right]=\hat{S}_{\hat{g}_{O}(\sigma) / \hat{h}_{O}(\sigma)}\left[\mathcal{M} \otimes \mathbb{1}_{2}, \hat{g}, \hat{h}_{ \pm} ; \Gamma_{\frac{1}{2}}\right]
\end{gathered}
$$

where the twisted gauge transformation matrix $\hat{\psi} \in \widehat{\mathfrak{H}}_{O}(\sigma)$ is any subgroup orbifold element. As seen in Eq. (5.44f), the general coset orientation-orbifold action is invariant under the general twisted vector gauge transformation. 
For the action of world-sheet parity on the twisted gauge fields and the twisted gauge transformation matrix, we find

$$
\begin{gathered}
\hat{A}_{ \pm}(\mathcal{T},-\xi)=\tau_{3} \hat{A}_{\mp}(\mathcal{T}, \xi) \tau_{3}, \quad \hat{h}_{ \pm}(\mathcal{T},-\xi)=\tau_{3} \hat{h}_{\mp}^{-1}(\mathcal{T}, \xi) \tau_{3} \\
\hat{\psi}(\mathcal{T},-\xi)=\tau_{3} \hat{\psi}(\mathcal{T}, \xi) \tau_{3}
\end{gathered}
$$

and each of the new twisted fields commutes with $\tau_{1}$, as usual. These commutators tell us that the new fields also have only two independent components in the two-dimensional space, for example:

$$
\begin{gathered}
\hat{A}_{ \pm}(\mathcal{T}, \xi, t, \sigma)=\left(\begin{array}{cc}
\hat{A}_{ \pm}^{(0)}(\mathcal{T}, \xi, t, \sigma) & \hat{A}_{ \pm}^{(1)}(\mathcal{T}, \xi, t, \sigma) \\
\hat{A}_{ \pm}^{(1)}(\mathcal{T}, \xi, t, \sigma) & \hat{A}_{ \pm}^{(0)}(\mathcal{T}, \xi, t, \sigma)
\end{array}\right) \\
\hat{A}_{ \pm}^{(u)}(\mathcal{T},-\xi, t, \sigma)=(-1)^{u} \hat{A}_{\mp}^{(u)}(\mathcal{T}, \xi, t, \sigma) .
\end{gathered}
$$

In the special case of the class $h_{\sigma}^{2}=1$, all the fields defined here have the usual monodromy

$$
\begin{gathered}
h_{\sigma}^{2}=1, E(T, \sigma)^{2}=1: \quad \hat{O}(\mathcal{T}, \xi+2 \pi, \sigma)=\tau_{3} E(T, \sigma) \hat{O}(\mathcal{T}, \xi, \sigma) E^{*}(T, \sigma) \tau_{3} \\
\hat{O}=\hat{g}, \hat{h}_{ \pm}, \hat{A}_{ \pm} \text {or } \hat{\psi}
\end{gathered}
$$

as given for $\hat{g}$ in Eq. (2.25b). It follows in particular that each term of each integrand of the coset orientation-orbifold action is $2 \pi$-periodic.

To explicitly construct the twisted sectors of particular coset orientation orbifolds, the standard procedure [23] is to incorporate the embedding $\widehat{\mathfrak{H}}_{O}(\sigma) \subset \widehat{\mathfrak{G}}_{O}(\sigma)$ at the tangentspace level

$$
\begin{gathered}
\hat{g}=e^{i \hat{x}^{n(r) \mu u} \mathcal{T}_{n(r) \mu u}} \longrightarrow \hat{h}=e^{i \hat{\beta}^{\hat{n}(r) \hat{\mu} u} \mathcal{T}_{\hat{n}(r) \hat{\mu} u}} \\
\hat{J}_{\hat{n}(r) \hat{\mu} u}(\xi, t, \sigma) \equiv R_{r}(\sigma)_{\hat{\mu}}{ }^{\mu} \hat{J}_{\hat{n}(r) \mu u}(\xi, t, \sigma), \quad u=0,1, \forall \hat{n}(r), \hat{\mu} \in \hat{h}_{O}(\sigma) \subset \hat{g}_{O}(\sigma) \\
\mathcal{T}_{\hat{n}(r) \hat{\mu} u}(T, \sigma) \equiv R_{r}(\sigma)_{\hat{\mu}}{ }^{\mu} \mathcal{T}_{\hat{n}(r) \mu u}(T, \sigma) \\
\{\hat{n}(r)\} \subset\{n(r)\}, \quad \operatorname{dim}\{\hat{\mu}\} \leq \operatorname{dim}\{\mu\}
\end{gathered}
$$

where $R_{r}(\sigma)$ at fixed $\hat{n}(r)$ is the embedding matrix of both $\hat{\mathfrak{h}}_{O}(\sigma) \subset \hat{\mathfrak{g}}_{O}(\sigma)$ and $\hat{h}_{O}(\sigma) \subset$ $\hat{g}_{O}(\sigma)$. We emphasize with Ref. [23] that the orbifold affine algebras and their corresponding orbifold Lie algebras

$$
\begin{aligned}
& {\left[\hat{J}_{\hat{\mathfrak{g}}_{O}(\sigma)}(\cdot), \hat{J}_{\hat{\mathfrak{g}}_{O}(\sigma)}(\cdot)\right]=i \mathcal{F}_{\hat{\mathfrak{g}}_{O}(\sigma)} \hat{J}_{\hat{\mathfrak{g}}_{O}(\sigma)}(\cdot)+\mathcal{G}_{\hat{\mathfrak{g}}_{O}(\sigma)} \longrightarrow\left[\mathcal{T}_{\hat{g}_{O}(\sigma)}, \mathcal{T}_{\hat{g}_{O}(\sigma)}\right]=i \mathcal{F}_{\hat{\mathfrak{g}}_{O}(\sigma)} \mathcal{T}_{\hat{g}_{O}(\sigma)}} \\
& {\left[\hat{J}_{\hat{\mathfrak{h}}_{O}(\sigma)}(\cdot), \hat{J}_{\hat{\mathfrak{h}}_{O}(\sigma)}(\cdot)\right]=i \mathcal{F}_{\hat{\mathfrak{h}}_{O}(\sigma)} \hat{J}_{\hat{\mathfrak{h}}_{O}(\sigma)}(\cdot)+\mathcal{G}_{\hat{\mathfrak{h}}_{O}(\sigma)} \longrightarrow\left[\mathcal{T}_{\hat{h}_{O}(\sigma)}, \mathcal{T}_{\hat{h}_{O}(\sigma)}\right]=i \mathcal{F}_{\hat{\mathfrak{h}}_{O}(\sigma)} \mathcal{T}_{\hat{h}_{O}(\sigma)}}
\end{aligned}
$$


share the same twisted structure constants $\mathcal{F}_{\hat{\mathfrak{g}}_{O}(\sigma)}$ and $\mathcal{F}_{\hat{\mathfrak{h}}_{O}(\sigma)}$.

We turn next to the variational boundary conditions implied by the coset orientationorbifold action. Assuming provisionally that the previous boundary conditions from the WZW orientation-orbifold action continue to hold, we find the following list of boundary conditions

$$
\begin{aligned}
& \text { at } \xi=0, \pi: \\
& K(\xi)=R^{\xi}(\xi)=0 \\
& K_{1}(\xi) \equiv \widehat{\operatorname{Tr}}\left(\left(\mathcal{M} \otimes \mathbb{1}_{2}\right)\left(\hat{g} \hat{A}_{+}+\hat{A}_{-} \hat{g}^{-1}\right) \delta \hat{g}\right)=0 \\
& B_{1}(\xi) \equiv \widehat{\operatorname{Tr}}\left(\left(\mathcal{M} \otimes \mathbb{1}_{2}\right) \hat{\psi}^{-1} \partial_{t} \hat{\psi}\left(\hat{g}^{-1} \delta \hat{g}+\delta \hat{g} \hat{g}^{-1}\right)\right)=0 \\
& B_{2}(\xi) \equiv \widehat{\operatorname{Tr}}\left(\left(\mathcal{M} \otimes \mathbb{1}_{2}\right) \hat{\psi}^{-1} \partial_{\xi} \hat{\psi}\left(\hat{g}^{-1} \delta \hat{g}-\delta \hat{g} \hat{g}^{-1}\right)\right)=0 \\
& B_{3}(\xi) \equiv \widehat{\operatorname{Tr}}\left(\left(\mathcal{M} \otimes \mathbb{1}_{2}\right)\left[\hat{g}^{-1}, \hat{\psi}^{-1} \partial_{\xi} \hat{\psi}\right]\left(\partial_{\rho} \hat{g} \hat{g}^{-1} \delta \hat{g}-\delta \hat{g} \hat{g}^{-1} \partial_{\rho} \hat{g}\right)\right)=0
\end{aligned}
$$

where we have neglected total time derivatives in the variation, as usual. The first two boundary conditions in Eq. (5.50a) were obtained earlier in Eqs. (3.12b) and (3.13d). The boundary condition on $K_{1}(\xi)$ in Eq. (5.50b) is obtained by varying the gauge terms in the coset action (5.43), while the boundary conditions on $B_{i}(\xi), i=1,2,3$ arise by requiring that the first three boundary conditions are gauge-invariant.

Using the world-sheet parities in Eqs. (2.21d) and (5.45), it is straightforward to show that all these quantities are odd under world-sheet parity

$$
K(-\xi)=-K(\xi), \quad R^{\xi}(-\xi)=-R^{\xi}(\xi), \quad K_{1}(-\xi)=-K_{1}(\xi), \quad B_{i}(-\xi)=-B_{i}(\xi)
$$

so that the variational boundary conditions in (5.50) at $\xi=0$ are automatically satisfied. Moreover, for the class $h_{\sigma}^{2}=1$, all these quantities have trivial monodromy and therefore all the boundary conditions (5.50) are likewise satisfied explicitly at $\xi=\pi$.

Similarly, we obtain the boundary conditions on the twisted gauge fields

$$
\begin{gathered}
\hat{A}_{-}^{(u)}(\mathcal{T}, 0)=(-1)^{u} \hat{A}_{+}^{(u)}(\mathcal{T}, 0) \\
h_{\sigma}^{2}=1, E(T, \sigma)^{2}=1: \quad \hat{A}_{-}^{(u)}(\mathcal{T}, \pi)=E^{*}(T, \sigma) \hat{A}_{+}^{(u)}(\mathcal{T}, \pi) E(T, \sigma)
\end{gathered}
$$

from the world-sheet parity (5.46b) and the monodromy (5.47). 
Our final task is to integrate out the twisted matrix gauge fields

$$
\begin{gathered}
\hat{A}_{+}(T, \xi, t, \sigma)=\hat{A}_{+}^{\hat{n}(r) \hat{\mu} u}(\xi, t, \sigma) \mathcal{T}_{\hat{n}(r) \hat{\mu} u}(T, \sigma) \in \hat{h}_{O}(\sigma) \\
\hat{A}_{-}(T, \xi, t, \sigma)=\hat{A}_{-}^{\hat{n}(r) \hat{\mu} u}(\xi, t, \sigma) \mathcal{T}_{\hat{n}(r) \hat{\mu} u}(T, \sigma)(-1)^{u} \\
\hat{A}_{-}^{\hat{n}(r) \hat{\mu} u}(\xi, t, \sigma)=\hat{A}_{+}^{\hat{n}(r) \hat{\mu} u}(-\xi, t, \sigma)
\end{gathered}
$$

to find the equivalent orientation-orbifold sigma-model form of the coset orientation-orbifold action. For this computation, we will need the definitions

$$
\begin{aligned}
& \mathcal{G}_{n(r) \mu u ; \hat{n}(s) \hat{\nu} v}(\sigma) \equiv \widehat{\operatorname{Tr}}\left(\left(\mathcal{M} \otimes \mathbb{1}_{2}\right) \mathcal{T}_{n(r) \mu u}(T, \sigma) \mathcal{T}_{\hat{n}(s) \hat{\nu} v}(T, \sigma)\right) \\
& =R_{s}(\sigma)_{\hat{\nu}}{ }^{\nu} \mathcal{G}_{n(r) \mu u ; \hat{n}(s) \nu v}(\sigma)=R_{s}(\sigma)_{\hat{\nu}}{ }^{\nu} \mathcal{G}_{\hat{n}(s) \nu v ; n(r) \mu u}(\sigma) \equiv \mathcal{G}_{\hat{n}(s) \hat{\nu} v ; n(r) \mu u}(\sigma) \\
& \mathcal{G}_{\hat{n}(r) \hat{\mu} u ; \hat{n}(s) \hat{\nu} v}(\sigma) \equiv \widehat{\operatorname{Tr}}\left(\left(\mathcal{M} \otimes \mathbb{1}_{2}\right) \mathcal{T}_{\hat{n}(r) \hat{\mu} u}(T, \sigma) \mathcal{T}_{\hat{n}(s) \hat{\nu} v}(T, \sigma)\right) \\
& =R_{r}(\sigma)_{\hat{\mu}}^{\mu} R_{s}(\sigma)_{\hat{\nu}}^{\nu} \mathcal{G}_{\hat{n}(r) \mu u ; \hat{n}(s) \nu v}(\sigma)=\mathcal{G}_{\hat{n}(s) \hat{\nu} v ; \hat{n}(r) \hat{\mu} u}(\sigma) \\
& \mathcal{G}_{\hat{n}(r) \hat{\mu} u ; n(s) \nu v}(\sigma)=\delta_{u+v, 0 \bmod 2} \delta_{\hat{n}(r)+n(s), 0 \bmod \rho(\sigma)} \mathcal{G}_{\hat{n}(r) \hat{\mu} u ;-\hat{n}(r), \nu,-u}(\sigma) \\
& \mathcal{G}_{\hat{n}(r) \hat{\mu} u ; \hat{n}(s) \hat{\nu} v}(\sigma)=\delta_{u+v, 0 \bmod 2} \delta_{\hat{n}(r)+\hat{n}(s), 0 \bmod \rho(\sigma)} \mathcal{G}_{\hat{n}(r) \hat{\mu} u ;-\hat{n}(r), \hat{\nu},-u}(\sigma)
\end{aligned}
$$

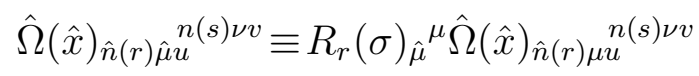

$$
\begin{aligned}
& \hat{\Omega}(\hat{x})_{\hat{n}(r) \hat{\mu} u ; \hat{n}(s) \hat{\nu} v} \equiv \hat{\Omega}(\hat{x})_{\hat{n}(r) \hat{\mu} u} u^{n(t) \delta w} \mathcal{G}_{n(t) \delta w ; \hat{n}(s) \hat{\nu} v}(\sigma)
\end{aligned}
$$

where $\mathcal{M}$ is the twisted data matrix in $(2.14 \mathrm{f}), \mathcal{G}_{\hat{\mathfrak{h}}_{O}(\sigma)} \simeq\left\{\mathcal{G}_{\hat{n}(r) \hat{\mu} u ; \hat{n}(s) \hat{\nu} v}(\sigma)\right\}$ is the induced tangent-space metric on the twisted affine subalgebra $\hat{\mathfrak{h}}_{O}(\sigma)$, and $\hat{\Omega}$ is the twisted adjoint action in Eq. (4.29). The induced metric $\mathcal{G}_{\hat{\mathfrak{h}}_{O}(\sigma)}$ is invertible in the $\hat{h}_{O}(\sigma)$ subspace

$$
\mathcal{G}_{\hat{n}(r) \hat{\mu} u ; \hat{n}(t) \hat{\delta} w}(\sigma) \mathcal{G}^{\hat{n}(t) \hat{\delta} w ; \hat{n}(s) \hat{\nu} v}(\sigma)=\delta_{\hat{\mu}}^{\hat{\nu}} \delta_{u-v, 0 \bmod 2} \delta_{\hat{n}(r)-\hat{n}(s), 0 \bmod \rho(\sigma)}
$$

because the original $h_{\sigma}$-symmetric coset construction $\frac{g}{h}\left(h_{\sigma}\right)$ was a reductive coset space.

Following the conventional procedure, we may then integrate out the twisted matrix gauge fields by solving their equations of motion:

$$
\begin{gathered}
\widehat{\operatorname{Tr}}\left\{\left(\mathcal{M} \otimes \mathbb{1}_{2}\right)\left(-i \partial_{-} \hat{g} \hat{g}^{-1}-\hat{g} \hat{A}_{-} \hat{g}^{-1}+\hat{A}_{-}\right) \mathcal{T}_{\hat{n}(r) \hat{\mu} u\}=0}\right. \\
\widehat{\operatorname{Tr}}\left\{\left(\mathcal{M} \otimes \mathbb{1}_{2}\right)\left(i \hat{g}^{-1} \partial_{+} \hat{g}-\hat{g}^{-1} \hat{A}_{+} \hat{g}+\hat{A}_{+}\right) \mathcal{T}_{\hat{n}(r) \hat{\mu} u}\right\}=0, \quad u=0,1, \quad \forall \hat{n}(r), \hat{\mu} \in \hat{h}_{O}(\sigma) .
\end{gathered}
$$


After some algebra, we find that open-string sector $\hat{h}_{\sigma}$ of the general coset orientation orbifold $A_{g / h}\left(H_{-}\right) / H_{-}$is described by the sigma model orientation-orbifold action (5.20) with the special values of the twisted Einstein metric $\hat{G}^{\text {tot }}$ and twisted $B$ field $\hat{B}^{\text {tot }}$ :

$$
\begin{aligned}
(\hat{G}(\hat{x})+\hat{B}(\hat{x}))_{n(r) \mu u ; n(s) \nu v}^{t o t}=(\hat{G}(\hat{x})+\hat{B}(\hat{x}))_{n(r) \mu u ; n(s) \nu v} & \\
- & 2 \hat{Z}(\hat{x})_{n(r) \mu u ; \hat{n}\left(r^{\prime}\right) \hat{\mu}^{\prime} u^{\prime}} \hat{\bar{Z}}(\hat{x})_{n(s) \nu v ; \hat{n}\left(s^{\prime}\right) \hat{\nu}^{\prime} v^{\prime}} \hat{X}^{-1}(\hat{x})^{\hat{n}\left(s^{\prime}\right) \hat{\nu}^{\prime} v^{\prime} ; \hat{n}\left(r^{\prime}\right) \hat{\mu}^{\prime} u^{\prime}} \\
& \hat{Z}(\hat{x})_{n(r) \mu u ; n(s) \nu v} \equiv \hat{e}(\hat{x})_{n(r) \mu u^{n(t) \delta w}} \mathcal{G}_{n(t) \delta w ; n(s) \nu v}(\sigma) \\
& \hat{Z}(\hat{x})_{n(r) \mu u ; n(s) \nu v} \equiv \hat{\bar{e}}(\hat{x})_{n(r) \mu u^{n(t) \delta w}} \mathcal{G}_{n(t) \delta w ; n(s) \nu v}(\sigma) \\
& \hat{X}_{\hat{n}(r) \hat{\mu} u ; \hat{n}(s) \hat{\nu} v}(\hat{x}) \equiv(\mathcal{G}(\sigma)-\hat{\Omega}(\hat{x}))_{\hat{n}(r) \hat{\mu} u ; \hat{n}(s) \hat{\nu} v} .
\end{aligned}
$$

Here the quantities $\hat{G}, \hat{B}, \hat{e}$ and $\hat{\Omega}$ are the WZW orientation-orbifold fields defined in Subsec. 5.3, and the quantity $\hat{\bar{e}}$ is given by:

$$
\begin{gathered}
-i \hat{g}(\mathcal{T}, \hat{x}) \hat{\partial}_{n(r) \mu u} \hat{g}^{-1}(\mathcal{T}, \hat{x})=\hat{e}(\hat{x})_{n(r) \mu u}{ }^{n(s) \nu v} \mathcal{T}_{n(s) \nu v} \\
\hat{\bar{e}}(\hat{x}(\xi))_{n(r) \mu u}{ }^{n(s) \nu v} \equiv-(\hat{e}(\hat{x}(\xi)) \hat{\Omega}(\hat{x}(\xi)))_{n(r) \mu u}{ }^{n(s) \nu v}=(-1)^{u+v+1} \hat{e}(\hat{x}(-\xi))_{n(r) \mu u}{ }^{n(s) \nu v} .
\end{gathered}
$$

The matrix $\hat{X}(\hat{x})$ in Eq. (5.57d) is invertible in the $\hat{h}_{O}(\sigma)$ subspace because the induced metric $\mathcal{G}_{\hat{\mathfrak{h}}_{O}(\sigma)} \simeq\left\{\mathcal{G}_{\hat{n}(r) \hat{\mu} u ; \hat{n}(s) \hat{\nu} v}(\sigma)\right\}$ is invertible. The same result (5.57) for the sigmamodel form of the coset orientation-orbifold action can be obtained by eigenfields and local isomorphisms from the sigma-model form of the coset action (see App. B).

The coset orientation-orbifold sigma models should be considered in fixed gauges such as:

$$
\begin{aligned}
0= & \widehat{\operatorname{Tr}}\left(\left(\mathcal{M} \otimes \mathbb{1}_{2}\right) \hat{x}_{\sigma}^{n(s) \nu v}(\xi, t) \mathcal{T}_{n(s) \nu v} \mathcal{T}_{\hat{n}(r) \hat{\mu} u}\right) \\
& =\hat{x}_{\sigma}^{n(s) \nu v} \mathcal{G}_{n(s) \nu v ; \hat{n}(r) \hat{\mu} u}(\sigma)=2 \hat{x}_{\sigma}^{-\hat{n}(r), \hat{\nu},-u} \mathcal{G}_{-\hat{n}(r), \hat{\nu} ; \hat{n}(r) \hat{\mu}}(\sigma) .
\end{aligned}
$$

The contribution of the orientation-orbifold dilaton to the coset orientation orbifolds is included in the discussion of Sec.6.

\subsection{Example: Twisted Open-String Free Bosons}

As another example in the sigma model, we consider the simple case of free bosons with a linear symmetry $\omega\left(h_{\sigma}\right)$

$$
\begin{gathered}
G_{i j}(x)=\text { const., } \quad G_{i j}=\omega\left(h_{\sigma}\right)_{i}{ }^{k} \omega\left(h_{\sigma}\right)_{j}{ }^{l} G_{k l}, \quad B_{i j}(x)=0 \\
S=\frac{1}{8 \pi} \int d t \int_{0}^{2 \pi} d \xi G_{i j} \partial_{+} x^{i} \partial_{-} x^{j}
\end{gathered}
$$


which may be considered as the abelian limit of the WZW models.

In open-string orientation-orbifold sector $\hat{h}_{\sigma}=\tau_{1} \times h_{\sigma}$, the results of the preceding subsections reduce to

$$
\begin{gathered}
\hat{G}_{n(r) \mu u ; n(s) \nu v}(\hat{x})=\text { const. } \\
=\delta_{n(r)+n(s), 0 \bmod \rho(\sigma)} \delta_{u+v, 0 \bmod 2} \hat{G}_{n(r) \mu ;-n(r), \nu}^{(0)} \\
\hat{G}_{n(r) \mu ; n(s) \nu}^{(0)}=2 \mathcal{G}_{n(r) \mu ; n(s) \nu}(\sigma) \\
\hat{B}_{n(r) \mu u ; n(s) \nu v}(\hat{x})=0, \quad \hat{x}_{\sigma}^{n(r) \mu u}(-\xi)=(-1)^{u+1} \hat{x}_{\sigma}^{n(r) \mu u}(\xi) \\
\hat{S}_{\sigma}=\frac{1}{8 \pi} \int d t \int_{0}^{\pi} d \xi \hat{G}_{n(r) \mu ;-n(r), \nu}^{(0)} \sum_{u=0}^{1} \partial_{+} \hat{x}^{n(r) \mu u} \partial_{-} \hat{x}^{-n(r), \nu,-u} \\
\Rightarrow \partial_{+} \partial_{-} \hat{x}^{n(r) \mu u}=0
\end{gathered}
$$

where $\hat{x}$ are the twisted open-string free bosons and $\mathcal{G}$ is the ordinary twisted tangent space metric in Eq. (2.19c) The variational boundary condition (5.22) takes the form

$$
\hat{G}_{n(r) \mu ;-n(r), \nu}^{(0)} \sum_{u=0}^{1} \partial_{\xi} \hat{x}^{n(r) \mu u} \delta \hat{x}^{-n(r), \nu,-u}=0 \text { at } \xi=0, \pi
$$

which also follows of course by variation of the action (5.61e). One way to consider these boundary conditions is to take $\delta \hat{x}$ infinitesimal and divide by a corresponding time increment $\delta t$ :

$$
\hat{G}_{n(r) \mu ;-n(r), \nu}^{(0)} \sum_{u=0}^{1} \partial_{\xi} \hat{x}^{n(r) \mu u} \partial_{t} \hat{x}^{-n(r), \nu,-u}=0 \text { at } \xi=0, \pi .
$$

Similarly, $\delta \hat{x}$ can be replaced by $\partial_{t} \hat{x}$ in the general boundary condition (15.22).

In our previous paper [25], we gave the correct coordinate boundary conditions or branes for these twisted bosons

$$
\begin{gathered}
\partial_{t} \hat{x}^{n(r) \mu 0}=\partial_{\xi} \hat{x}^{n(r) \mu 1}=0 \text { at } \xi=0 \\
\partial_{t} \hat{x}^{n(r) \mu u}=i \tan \left(\frac{n(r)}{\rho(\sigma)} \pi\right) \partial_{\xi} \hat{x}^{n(r) \mu u} \text { at } \xi=\pi
\end{gathered}
$$

as well as the corresponding field expansions and quasi-canonical algebra. The boundary conditions (5.64) were derived from the equations of motion

$\partial_{+} \hat{x}^{n(r) \mu u}=2 \mathcal{G}^{n(r) \mu u ; n(s) \nu v}(\sigma) \hat{J}_{n(s) \nu v}(\xi, t), \quad \partial_{-} \hat{x}^{n(r) \mu u}=2 \mathcal{G}^{n(r) \mu u ; n(s) \nu v}(\sigma) \hat{J}_{n(s) \nu v}(-\xi, t)$ 
using the monodromies of the currents - which are known from the operator theory. This situation is analogous to that which was encountered for WZW orientation orbifolds in Eq. (3.21).

At $\xi=0$, these coordinate boundary conditions are the same as those which followed from world-sheet parity above, and they correspondingly satisfy the variational boundary condition (5.63) at $\xi=0$.

The coordinate boundary condition (5.64b also solves the variational boundary condition (15.63) at $\xi=\pi$. To see this, one needs only the identity

$$
\hat{G}_{n(r) \mu ;-n(r), \nu}^{(0)} \tan \left(\frac{n(r)}{\rho(\sigma)} \pi\right) \sum_{u=0}^{1} \partial_{\xi} \hat{x}^{n(r) \mu u} \partial_{\xi} \hat{x}^{-n(r), \nu,-u}=0
$$

which follows because the tangent is odd under the relabelling $n(r) \mu u \leftrightarrow-n(r), \nu,-u$ of the dummy indices.

Although mixed boundary conditions such as Eq. (5.64b) are conventionally associated to non-zero $B$ field backgrounds, we emphasize that there is no twisted $\hat{B}$ field in the background of these twisted open-string free bosons.

\section{The Orientation-Orbifold Einstein Equations}

In analogy to the Einstein equations $[44-49,13]$ of the untwisted sigma model $A_{M}$ and the twisted Einstein equations [24] of the ordinary sigma-model orbifold $A_{M}\left(H_{+}\right) / H_{+}$, we will now construct the twisted Einstein equations of open-string sector $\hat{h}_{\sigma}$ of the sigma-model orientation orbifold $A_{M}\left(H_{-}\right) / H_{-}$.

\subsection{The Two-Component Form of the Ordinary Einstein Equations}

We begin this construction ${ }^{\ddagger 8}$ with the ordinary Einstein equations $[44-49,13]$

$$
\begin{gathered}
R_{i j}(x)+\frac{1}{4} H_{k i}^{l}(x) H_{l j}^{k}(x)-2 \nabla_{i} \phi_{j}(x)=0 \\
\left(\nabla_{k}-2 \phi_{k}(x)\right) H^{k}{ }_{i j}(x)=0
\end{gathered}
$$

which describe one-loop conformal sigma models in the presence of a general dilaton vector $\phi_{i}$ (see e. g. Ref. [13]). The special case of the ordinary dilaton solution is

$$
\phi_{i}=\nabla_{i} \phi=\partial_{i} \phi
$$

\footnotetext{
${ }^{\ddagger 8}$ The Riemann and Ricci tensor conventions of Ref. [13] are followed here.
} 
where $\phi$ is the dilaton.

We first ask which of these Einstein systems is invariant under the basic orientation reversal

$$
x^{i}(\xi)^{\prime}=-x^{i}(-\xi), \quad O(x)^{\prime}=O\left(x^{\prime}\right)
$$

where $O$ includes any tensor. To compare the original Einstein system (6.1) with the image of the system under orientation reversal, we need the basic space-time parities

$$
G_{i j}(-x)=G_{i j}(x), \quad B_{i j}(-x)=-B_{i j}(x), \quad H_{i j k}(-x)=H_{i j k}(x)
$$

(which we already know from the invariance of the sigma-model action) as well as the derived space-time parities

$$
\begin{gathered}
G^{i j}(-x)=G^{i j}(x), \quad H_{i j}{ }^{k}(-x)=H_{i j}{ }^{k}(x) \\
\Gamma_{i j}{ }^{k}(-x)=-\Gamma_{i j}{ }^{k}(x), \quad \nabla_{i}(-x)=-\nabla_{i}(x) \\
R_{i j k l}(-x)=R_{i j k l}(x),
\end{gathered}
$$

which follow from the standard formulae of Riemannian geometry. Then we find that the necessary and sufficient condition for invariance of the Einstein system is the following space-time parity of the dilaton vector:

$$
\phi_{i}(-x)=-\phi_{i}(x) \longrightarrow \phi(-x)=\phi(x)
$$

We recall that the space-time parities (6.4) were verified in Subsec. 4.1 for WZW models and principal chiral models. Appendix B also shows that the space-time parities in Eqs. (6.4), (6.5) hold for all (reductive) coset CFTs.

As discussed for the sigma-model action in Subsec. 5.1, the space-time parities above allow us to rewrite the Einstein system in the two-component notation:

$$
\begin{gathered}
R_{i \dot{I} ; j \dot{J}}(x)+{ }^{\frac{1}{4}} H_{k \dot{K} ; i \dot{I}}{ }^{l \dot{L}}(x) H^{k \dot{K}}{ }_{l \dot{L} ; j \dot{J}}(x)-2 \nabla_{i \dot{I}} \phi_{j \dot{J}}(x)=0 \\
\left(\nabla_{k \dot{K}}-2 \phi_{k \dot{K}}(x)\right) H^{k \dot{K}}{ }_{i \dot{I} ; j \dot{J}}(x)=0 \\
x^{i \dot{I}}(\xi)=(-1)^{\dot{I}} x^{i}\left((-1)^{\dot{I}} \xi\right), \quad \partial_{i \dot{I}}=\frac{\partial}{\partial x^{i \dot{I}}}, \quad \dot{I}=0,1 \\
G_{i \dot{I} ; j \dot{J}}(x)=\delta_{\dot{I} \dot{j}} G_{i j}\left(x^{\dot{I}}\right), \quad B_{i \dot{I} ; j \dot{J}}(x)=\delta_{\dot{I} \dot{J}} B_{i j}\left(x^{\dot{I}}\right) \\
H_{i \dot{I} ; j \dot{J} ; k \dot{K}}(x)=\partial_{i \dot{I}} B_{j \dot{j} ; k \dot{K}}(x)+\operatorname{cyclic}=\delta_{\dot{I} \dot{J}} \delta_{\dot{j} \dot{K}} H_{i j k}\left(x^{\dot{I}}\right) \\
\phi_{i \dot{I}}(x) \equiv \phi_{i}\left(x^{\dot{I}}\right) \rightarrow \frac{\partial}{\partial x^{i \dot{I}}} \phi\left(x^{\dot{I}}\right) .
\end{gathered}
$$


In the two-component notation, we assume all the standard formulae of Riemannian geometry with $i \rightarrow i \dot{I}$ for every index - for example:

$$
\begin{gathered}
G^{i \dot{I} ; j \dot{J}}(x)=\delta^{\dot{I}} G^{i j}\left(x^{\dot{I}}\right) \\
\Gamma_{i \dot{I} ; j \dot{J}}{ }^{k \dot{K}}(x) \equiv \frac{1}{2}\left(\partial_{i \dot{I}} G_{j \dot{j} ; l \dot{L}}(x)+\partial_{j \dot{j}} G_{i \dot{I} ; l \dot{L}}(x)-\partial_{l \dot{L}} G_{i \dot{I} ; j \dot{J}}(x)\right) G^{i \dot{L} ; k \dot{K}}(x) \\
=\delta_{\dot{I} \dot{j}} \delta_{\dot{J}}^{\dot{K}} \Gamma_{i j}{ }^{k}\left(x^{\dot{I}}\right) \\
\nabla_{i \dot{I}} \phi_{j \dot{J}}(x) \equiv \partial_{i \dot{I}} \phi_{j \dot{J}}(x)-\Gamma_{i \dot{I} ; j \dot{J}}^{k \dot{K}}(x) \phi_{k \dot{K}}(x)=\delta_{\dot{I} \dot{J}}\left(\partial_{i \dot{I}} \phi_{j}\left(x^{\dot{I}}\right)-\Gamma_{i j}{ }^{k}\left(x^{\dot{I}}\right) \phi_{k}\left(x^{\dot{I}}\right)\right) \\
R_{i \dot{I} ; \dot{j} ; k \dot{K} ; l \dot{L}}(x)=\delta_{\dot{I} \dot{j}} \delta_{\dot{J} \dot{K}} \delta_{\dot{K} \dot{L}} R_{i j k l}\left(x^{\dot{I}}\right), \quad R_{i \dot{I} ; j \dot{J}}(x)=\delta_{\dot{I} \dot{J}} R_{i j}\left(x^{\dot{I}}\right) .
\end{gathered}
$$

More generally, the two-component analogue of any Einstein field $O$ has the form

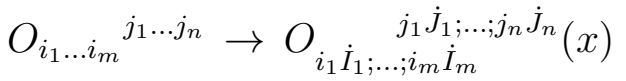

$$
\begin{aligned}
& O_{i_{1} \dot{I}_{1} ; \ldots ; i_{m} \dot{I}_{m}}^{j_{1} \dot{J}_{1} ; \ldots ; j_{n} \dot{J}_{n}}(x)=\delta_{\dot{I}_{1} \dot{I}_{2}} \ldots \delta_{\dot{I}_{m-1} \dot{I}_{m}} \delta_{\dot{I}_{m}}^{\dot{J}_{1}} \delta^{\dot{J}_{1} \dot{J}_{2}} \ldots \delta^{\dot{J}_{n-1} \dot{J}_{n}} O_{i_{1} \ldots i_{m}}^{j_{1} \ldots j_{n}}\left(x^{\dot{I}_{1}}\right)
\end{aligned}
$$

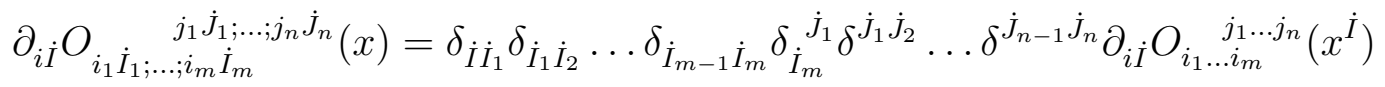

where the Kronecker deltas in these results set all $\dot{I}, \dot{J}$ indices equal.

In this formulation, the action of the basic orientation-reversing automorphism involves one $\tau_{1}$ for each $\dot{I}$ index. This includes the transformations in Eq. (5.8) and for example:

$$
\begin{gathered}
x^{i \dot{I}}(\xi)^{\prime}=-x^{i \dot{J}}(\xi)\left(\tau_{1}\right)_{\dot{J}}^{\dot{I}}, \quad A(x)^{\prime}=A\left(x^{\prime}\right) \\
\Gamma_{i \dot{I} ; j \dot{J}}^{k \dot{K}}(x)^{\prime}=\Gamma_{i \dot{I} ; j \dot{J}}{ }^{k \dot{K}}\left(x^{\prime}\right)=\left(\tau_{1}\right)_{\dot{I}}^{\dot{L}}\left(\tau_{1}\right)_{\dot{J}}{ }^{\dot{M}} \Gamma_{i \dot{L} ; j \dot{M}}{ }^{k \dot{N}}(x)\left(\tau_{1}\right)_{\dot{N}} \dot{K} \\
R_{i \dot{I} ; j \dot{J}}(x)^{\prime}=R_{i \dot{I} ; j \dot{J}}\left(x^{\prime}\right)=\left(\tau_{1}\right)_{\dot{I}}^{\dot{K}}\left(\tau_{1}\right)_{\dot{J}}^{\dot{L}} R_{i \dot{K} ; j \dot{L}}(x) .
\end{gathered}
$$

The invariance of the two-component Einstein system in (6.7) under the basic orientationreversing automorphism is now immediately transparent.

The space-time parities of the two-component fields

$$
\begin{gathered}
G_{i \dot{I} ; j \dot{J}}(-x)=G_{i \dot{I} ; j \dot{J}}(x), \quad B_{i \dot{I} ; j \dot{J}}(-x)=-B_{i \dot{I} ; j \dot{J}}(x) \\
H_{i \dot{I} ; j \dot{j} ; k \dot{K}}(-x)=H_{i \dot{I} ; j \dot{j} ; k \dot{K}}(x) \\
\Gamma_{i \dot{I} ; j \dot{j}}{ }^{k \dot{K}}(-x)=-\Gamma_{i \dot{I} ; j \dot{J}}{ }^{k \dot{K}}(x), \quad \nabla_{i \dot{I}}(-x)=-\nabla_{i \dot{I}}(x) \\
R_{i \dot{I} ; j \dot{J}}(-x)=R_{i \dot{I} ; j \dot{J}}(x), \quad \phi_{i \dot{I}}(-x)=-\phi_{i \dot{I}}(x)
\end{gathered}
$$

follow from Eqs. (6.4)-(6.5), and in fact form a symmetry of the two-component Einstein system (6.7). We emphasize however that the space-time parity is not a symmetry of the 
sigma-model action (5.1a), just as electromagnetic duality is not a symmetry of the Maxwell action.

The world-sheet parities of the two-component fields include those in Eq. (5.7) and

$$
\begin{aligned}
& G^{i \dot{I} ; j \dot{J}}(x(-\xi))=G^{i \dot{K} ; j \dot{L}}(x(\xi))\left(\tau_{1}\right)_{\dot{K}}^{\dot{I}}\left(\tau_{1}\right)_{\dot{L}}^{\dot{J}} \\
& H_{i \dot{I} ; j \dot{J}}{ }^{k \dot{K}}(x(-\xi))=\left(\tau_{1}\right)_{\dot{I}}^{\dot{L}}\left(\tau_{1}\right)_{\dot{J}}^{\dot{M}} H_{i \dot{L} ; j \dot{M}}{ }^{k \dot{N}}(x(\xi))\left(\tau_{1}\right)_{\dot{N}}^{\dot{K}} \\
& \Gamma_{i \dot{I} ; j \dot{j}}{ }^{k \dot{K}}(x(-\xi))=-\left(\tau_{1}\right)_{\dot{I}}^{\dot{L}}\left(\tau_{1}\right)_{\dot{J}}^{\dot{M}} \Gamma_{i \dot{L} ; j \dot{M}}{ }^{k \dot{N}}(x(\xi))\left(\tau_{1}\right)_{\dot{N}}^{\dot{K}} \\
& \nabla_{i \dot{I}}(-\xi)=-\left(\tau_{1}\right)_{\dot{I}}^{\dot{J}} \nabla_{i \dot{J}}(\xi), \quad R_{i \dot{I} ; j \dot{j}}(x(-\xi))=\left(\tau_{1}\right)_{\dot{I}}^{\dot{K}}\left(\tau_{1}\right)_{\dot{J}}^{\dot{L}} R_{i \dot{K} ; j \dot{L}}(x(\xi)) \\
& R_{i \dot{I} ; j \dot{J} ; k \dot{K} ; l \dot{L}}(x(-\xi))=\left(\tau_{1}\right)_{\dot{I}}^{\dot{M}}\left(\tau_{1}\right)_{\dot{J}}^{\dot{N}}\left(\tau_{1}\right)_{\dot{K}}^{\dot{P}}\left(\tau_{1}\right)_{\dot{L}}{ }^{\dot{Q}} R_{i \dot{M} ; j \dot{N} ; k \dot{P} ; l \dot{Q}}(x(\xi)) \\
& \phi_{i \dot{I}}(x(-\xi))=-\left(\tau_{1}\right)_{\dot{I}}^{j} \phi_{i \dot{j}}(x(\xi)) \longrightarrow \phi\left(x^{\dot{I}}(-\xi)\right)=\phi\left(x^{\dot{J}}(\xi)\left(\tau_{1}\right)_{\dot{J}}^{\dot{I}}\right)
\end{aligned}
$$

which follow from the definitions of the two-component objects. The general rule for the world-sheet parities is one $\tau_{1}$ for each $\dot{I}$ index and an extra minus sign when the space-time parity is odd.

We turn next to the action of the general orientation-reversing automorphism $\hat{h}_{\sigma}$ on the two-component fields. For $x, G, B$ and $H$, this action is given in Eq. (5.9), and the actions on the other fields

$$
\begin{gathered}
x^{i \dot{I}}(\xi)^{\prime}=x^{j \dot{J}}(\xi) \omega^{\dagger}\left(h_{\sigma}\right)_{j}{ }^{i}\left(\tau_{1}\right)_{\dot{J}}{ }^{\dot{I}}, \quad A(x)^{\prime}=A\left(x^{\prime}\right) \\
\Gamma_{i \dot{I} ; j \dot{J}}{ }^{k \dot{K}}(x)^{\prime}=\Gamma_{i \dot{I} ; j \dot{J}}{ }^{k \dot{K}}\left(x^{\prime}\right)=\omega\left(h_{\sigma}\right)_{i}{ }_{i}^{l} \omega\left(h_{\sigma}\right)_{j}{ }^{m}\left(\tau_{1}\right)_{\dot{I}}{ }^{\dot{L}}\left(\tau_{1}\right)_{\dot{J}}{ }^{\dot{M}} \Gamma_{l \dot{L} ; m \dot{M}}{ }^{n \dot{N}}(x) \omega^{\dagger}\left(h_{\sigma}\right)_{n}{ }^{k}\left(\tau_{1}\right)_{\dot{N}}{ }^{\dot{K}} \\
R_{i \dot{I} ; j \dot{j} ; k \dot{K} ; l \dot{L}}(x)^{\prime}=R_{i \dot{I} ; j \dot{J} ; k \dot{K} ; l \dot{L}}\left(x^{\prime}\right) \\
=\omega\left(h_{\sigma}\right)_{i}{ }^{m} \omega\left(h_{\sigma}\right)_{j}{ }^{n} \omega\left(h_{\sigma}\right)_{k}{ }^{p} \omega\left(h_{\sigma}\right)_{l}{ }^{q}\left(\tau_{1}\right)_{\dot{I}}{ }^{\dot{M}}\left(\tau_{1}\right)_{\dot{J}}{ }^{\dot{N}}\left(\tau_{1}\right)_{\dot{K}}{ }^{\dot{P}}\left(\tau_{1}\right)_{\dot{L}}{ }^{\dot{Q}} R_{m \dot{M} ; n \dot{N} ; p \dot{P} ; q \dot{Q}}(x) \\
R_{i \dot{I} ; j \dot{J}}(x)^{\prime}=R_{i \dot{I} ; j \dot{j}}\left(x^{\prime}\right)=\omega\left(h_{\sigma}\right)_{i}{ }^{k} \omega\left(h_{\sigma}\right)_{j}{ }^{l}\left(\tau_{1}\right)_{\dot{I}}{ }^{\dot{K}}\left(\tau_{1}\right)_{\dot{J}}{ }^{\dot{L}} R_{k \dot{K} ; l \dot{L}}(x)
\end{gathered}
$$

follow by the standard formulae of Riemannian geometry. With the corresponding transformation of the dilaton vector

$$
\phi_{i \dot{I}}(x)^{\prime}=\phi_{i \dot{I}}\left(x^{\prime}\right)=\omega\left(h_{\sigma}\right)_{i}{ }^{j}\left(\tau_{1}\right)_{\dot{I}}^{j} \phi_{j \dot{j}}(x)
$$

we find that the two-component Einstein system (6.7) is covariant under this automorphism. The general rule for the transformation of the Einstein fields is a factor $\omega \tau_{1}$ for each down index and a factor $\omega^{\dagger} \tau_{1}$ for each up index.

As above, the space-time parities (6.11) can be used to see that the linear symmetry conditions (5.10) for $G, B$ and $H$, as well as the corresponding linear symmetry conditions 
for the other fields

$$
\begin{gathered}
G^{i j}\left(x \omega^{\dagger}\right)=G^{k l}(x)\left(\omega^{\dagger}\right)_{k}{ }^{i}\left(\omega^{\dagger}\right)_{l}{ }^{j} \\
\Gamma_{i j}{ }^{k}\left(x \omega^{\dagger}\right)=\omega_{i}{ }^{l} \omega_{j}{ }^{m} \Gamma_{l m}{ }^{n}(x)\left(\omega^{\dagger}\right)_{n}{ }^{k} \\
R_{i j k l}\left(x \omega^{\dagger}\right)=\omega_{i}{ }^{m} \omega_{j}{ }^{n} \omega_{k}{ }^{p} \omega_{l}^{q} R_{m n p q}(x), \quad R_{i j}\left(x \omega^{\dagger}\right)=\omega_{i}{ }^{k} \omega_{j}{ }^{l} R_{k l}(x) \\
\phi_{i}\left(x \omega^{\dagger}\right)=\omega_{i}^{j} \phi_{j}(x) \longrightarrow \phi\left(x \omega^{\dagger}\right)=\phi(x)
\end{gathered}
$$

are incorporated in the transformations (6.13) and (6.14). The general rule for the linear symmetry conditions involves one $\omega$ for each down index and one $\omega^{\dagger}$ for each up index.

\subsection{Riemannian Eigenfields}

Using the eigenvector matrix of the Einstein-space $H$-eigenvalue problem (15.13), we next define the corresponding Riemannian eigenfields

$$
\begin{aligned}
& X_{\sigma}^{n(r) \mu u}(\xi) \equiv x^{i \dot{I}}(\xi) \chi(\sigma)_{n(r) \mu}^{-1} U^{\dagger}(\sigma)_{i}^{n(r) \mu}\left(\frac{1}{\sqrt{2}} U^{\dagger} \dot{I}^{u}\right), \quad \bar{u}=0,1 \\
& \mathcal{G}_{n(r) \mu u ; n(s) \nu v}(X) \equiv \chi(\sigma)_{n(r) \mu} \chi(\sigma)_{n(s) \nu} U(\sigma)_{n(r) \mu}{ }^{i} U(\sigma)_{n(s) \nu}{ }^{j}\left(\sqrt{2} U_{u}^{\dot{I}}\right)\left(\sqrt{2} U_{v}^{\dot{J}}\right) G_{i \dot{I} ; j \dot{j}}(x(X)) \\
& \mathcal{B}_{n(r) \mu u ; n(s) \nu v}(X) \equiv \chi(\sigma)_{n(r) \mu} \chi(\sigma)_{n(s) \nu} U(\sigma)_{n(r) \mu}{ }^{i} U(\sigma)_{n(s) \nu}{ }^{j}\left(\sqrt{2} U_{u}^{\dot{I}}\right)\left(\sqrt{2} U_{v}^{\dot{J}}\right) B_{i \dot{I} ; j \dot{J}}(x(X)) \\
& \mathcal{H}_{n(r) \mu u ; n(s) \nu v ; n(t) \delta w}(\mathcal{X}) \equiv \chi(\sigma)_{n(r) \mu} \chi(\sigma)_{n(s) \nu} \chi(\sigma)_{n(t) \delta} U(\sigma)_{n(r) \mu}{ }^{i} U(\sigma)_{n(s) \nu}{ }^{j} U(\sigma)_{n(t) \delta}{ }^{k} \\
& \times\left(\sqrt{2} U_{u}^{\dot{I}}\right)\left(\sqrt{2} U_{v}^{\dot{j}}\right)\left(\sqrt{2} U_{w}^{\dot{K}}\right) H_{i \dot{I} ; j \dot{j} ; k \dot{K}}(x(x)) \\
& \gamma_{n(r) \mu u ; n(s) \nu v}{ }^{n(t) \delta w}(X) \equiv \chi_{n(r) \mu} \chi_{n(s) \nu} U_{n(r) \mu}^{i} U_{n(s) \nu}^{j}\left(\sqrt{2} U_{u}^{\dot{I}}\right)\left(\sqrt{2} U_{v}^{\dot{J}}\right) \times \\
& \times \Gamma_{i \dot{I} ; j \dot{j}} \dot{k}^{\dot{K}}(x(X)) \chi_{n(t) \delta}^{-1}\left(U^{\dagger}\right)_{k}^{n(t) \delta}\left(\frac{1}{\sqrt{2}} U_{\dot{K}^{\dagger}}{ }^{w}\right) \\
& \mathcal{R}_{n(r) \mu u ; n(s) \nu v}(X) \equiv \chi_{n(r) \mu} \chi_{n(s) \nu} U_{n(r) \mu}{ }^{i} U_{n(s) \nu}{ }^{j}\left(\sqrt{2} U_{u}{ }^{\dot{I}}\right)\left(\sqrt{2} U_{v}{ }^{j}\right) R_{i \dot{I} ; j \dot{J}}(x(X)) \\
& \Phi_{n(r) \mu u}(\mathcal{X}) \equiv \chi_{n(r) \mu} U_{n(r) \mu}^{i}\left(\sqrt{2} U_{u}^{\dot{I}}\right) \phi_{i \dot{I}}(x(\mathcal{X})), \quad \bar{u}, \bar{v}, \bar{w} \in\{0,1\}
\end{aligned}
$$

where the $\chi$ 's are arbitrary normalization constants. The general rule for the eigenfields is a factor $\chi U(\sigma) \sqrt{2} U$ for each down index and a factor $\chi^{-1} U^{\dagger}(\sigma) \frac{1}{\sqrt{2}} U^{\dagger}$ for each up index. This prescription diagonalizes the automorphic response of all the eigenfields, for example: 


$$
\begin{gathered}
\gamma_{n(r) \mu u ; n(s) \nu v}{ }^{n(t) \delta w}(X)^{\prime}=e^{-2 \pi i\left(\frac{n(r)+n(s)-n(t)}{\rho(\sigma)}+\frac{u+v-w}{2}\right)} \gamma_{n(r) \mu u ; n(s) \nu v}{ }^{n(t) \delta w}(X) \\
\mathcal{R}_{n(r) \mu u ; n(s) \nu v ; n(t) \delta w ; n(q) \epsilon y}(X)^{\prime}= \\
=e^{-2 \pi i\left(\frac{n(r)+n(s)+n(t)+n(q)}{\rho(\sigma)}+\frac{u+v+w+y}{2}\right)} \mathcal{R}_{n(r) \mu u ; n(s) \nu v ; n(t) \delta w ; n(q) \epsilon y}(X) \\
\mathcal{R}_{n(r) \mu u ; n(s) \nu v}(X)^{\prime}=e^{-2 \pi i\left(\frac{n(r)+n(s)}{\rho(\sigma)}+\frac{u+v}{2}\right)} \mathcal{R}_{n r m u ; n(s) \nu v}(X) \\
\Phi_{n(r) \mu u}(X)^{\prime}=e^{-2 \pi i\left(\frac{n(r)}{\rho(\sigma)}+\frac{u}{2}\right)} \Phi_{n(r) \mu u}(X)
\end{gathered}
$$

The general rule for the automorphic responses of the eigenfields is a negative (positive) phase for each down (up) index.

As above, these eigenfields are two-component fields in the two-dimensional space. For example one has the forms of $\mathcal{G}, \mathcal{B}$ and $\mathcal{H}$ in (4.21), as well as:

$$
\begin{aligned}
\mathcal{G}^{n(r) \mu u ; n(s) \nu v}(X) & =\mathcal{G}_{(u+v)}^{n(r) \mu ; n(s) \nu}(X) \\
\gamma_{n(r) \mu u ; n(s) \nu v}{ }^{n(t) \delta w}(X) & =\gamma_{n(r) \mu ; n(s) \nu}^{(u+v-w)}{ }^{n(t) \delta}(X) \\
\mathcal{R}_{n(r) \mu u ; n(s) \nu v ; n(t) \delta w ; n(q) \epsilon y}(X) & =\mathcal{R}_{n(r) \mu ; n(s) \nu ; n(t) \delta ; n(q) \epsilon}^{(u+v+w+y)}(X) \\
\mathcal{R}_{n(r) \mu u ; n(s) \nu v}(X) & \mathcal{R}_{n(r) \mu ; n(s) \nu}^{(u+v)}(X)
\end{aligned}
$$

These relations are special cases of the general eigenfield identities

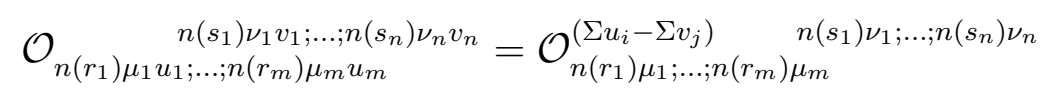

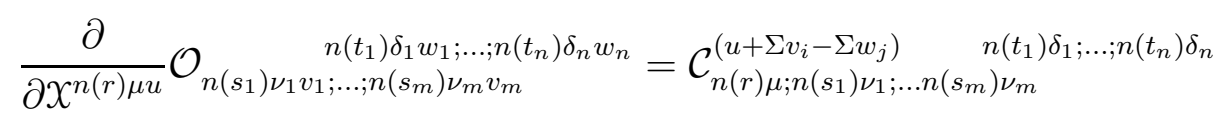

which follow from Eq. (6.9) and show the two-component structure for any eigenfield $\mathcal{O}$.

\subsection{Open-String Twisted Riemannian Geometry}

We then use the principle of local isomorphisms

$$
\begin{gathered}
x \underset{\sigma}{\longrightarrow} \hat{x}, \quad \mathcal{G} \underset{\sigma}{\longrightarrow} \hat{G}, \quad \mathcal{B} \underset{\sigma}{\longrightarrow} \hat{B}, \quad \mathcal{H} \underset{\sigma}{\longrightarrow} \hat{H} \\
\gamma \underset{\sigma}{\longrightarrow} \hat{\Gamma}, \quad \mathcal{R} \underset{\sigma}{\longrightarrow} \hat{R}, \quad \Phi \underset{\sigma}{\longrightarrow} \hat{\phi}
\end{gathered}
$$


to obtain the corresponding twisted fields in the open-string sectors. Relations among the twisted fields include

$$
\begin{array}{r}
\hat{\Gamma}_{n(r) \mu u ; n(s) \nu v}{ }^{n(t) \delta w}(\hat{x})=\frac{1}{2}\left(\hat{\partial}_{n(r) \mu u} \hat{G}_{n(s) \nu v ; n(q) \epsilon x}(\hat{x})+\hat{\partial}_{n(s) \nu v} \hat{G}_{n(r) \mu u ; n(q) \epsilon x}(\hat{x})\right. \\
\left.-\hat{\partial}_{n(q) \epsilon x} \hat{G}_{n(r) \mu u ; n(s) \nu v}(\hat{x})\right) \hat{G}^{n(q) \epsilon x ; n(t) \delta w}(\hat{x}) \\
\hat{\nabla}_{n(r) \mu u} \hat{\phi}_{n(s) \nu v}(\hat{x})=\hat{\partial}_{n(r) \mu u} \hat{\phi}_{n(s) \nu v}(\hat{x})-\hat{\Gamma}_{n(r) \mu u ; n(s) \nu v} n(t) \delta w \\
(\hat{x}) \hat{\phi}_{n(t) \delta w}(\hat{x})
\end{array}
$$

where $\hat{\Gamma}$ is the twisted Christoffel symbol and $\hat{\phi}$ is the orientation-orbifold dilaton vector. The twisted Riemann tensor is constructed as usual from the twisted Christoffel symbol.

We note that the twisted Christoffel symbols appear in the classical bulk equations of motion

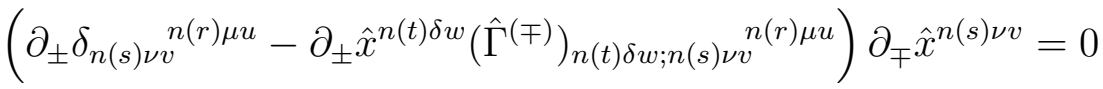

$$
\begin{aligned}
& \hat{\Gamma}^{( \pm)} \equiv \hat{\Gamma} \pm \frac{1}{2} \hat{H}
\end{aligned}
$$

which follow, as promised in Subsec. 5.3, by variation of the sigma-model orientationorbifold action.

Further applying the principle of local isomorphisms, we now discuss other properties of the twisted fields, beginning with the space-time parities

$$
\begin{gathered}
\qquad \hat{O}_{ \pm}(-\hat{x})= \pm \hat{O}_{ \pm}(\hat{x}) \\
\hat{O}_{+}=\hat{G}, \hat{H} \text { and the } \hat{R}^{\prime} s, \quad \hat{O}_{-}=\hat{x}, \hat{B}, \hat{\Gamma} \text { and } \hat{\phi}_{n(r) \mu u}
\end{gathered}
$$

which are the same as those of the corresponding untwisted fields. 
Similarly, each twisted field has only two independent reduced components

$$
\begin{aligned}
& \hat{\partial}_{n(r) \mu u}(\xi)=\frac{\partial}{\partial \hat{x}^{n(r) \mu u}(\xi)}, \quad \hat{\partial}_{n(r) \mu u}(\xi) \hat{x}_{\sigma}^{n(s) \nu v}(\xi)=\delta_{n(r)-n(s), 0 \bmod \rho(\sigma)} \delta_{\mu}{ }^{\nu} \delta_{u-v, 0 \bmod 2} \\
& \hat{G}_{n(r) \mu u ; n(s) \nu v}(\hat{x})=\hat{G}_{n(r) \mu ; n(s) \nu}^{(u+v)}(\hat{x}) \\
& \hat{B}_{n(r) \mu u ; n(s) \nu v}(\hat{x})=\hat{B}_{n(r) \mu ; n(s) \nu}^{(u+v)}(\hat{x}) \\
& \hat{H}_{n(r) \mu u ; n(s) \nu v ; n(t) \delta w}(\hat{x})=\hat{H}_{n(r) \mu ; n(s) \nu ; n(t) \delta}^{(u+v+w)}(\hat{x}) \\
& \hat{\Gamma}_{n(r) \mu u ; n(s) \nu v}^{n(t) \delta w}(\hat{x})=\hat{\Gamma}_{n(r) \mu ; n(s) \nu}^{(u+v-w)} n(t) \delta(\hat{x}) \\
& \hat{R}_{n(r) \mu u ; n(s) \nu v ; n(t) \delta w ; n(q) \epsilon y}(\hat{x})=\hat{R}_{n(r) \mu ; n(s) \nu ; n(t) \delta ; n(q) \epsilon}^{(u+v+w+y)}(\hat{x}) \\
& \hat{R}_{n(r) \mu u ; n(s) \nu v}(\hat{x})=\hat{R}_{n(r) \mu ; n(s) \nu}^{(u+v)}(\hat{x}) \\
& \hat{O}_{n\left(r_{1}\right) \mu_{1} u_{1} ; \ldots ; n\left(r_{m}\right) \mu_{m} u_{m}}^{n\left(s_{1}\right) \nu_{1} v_{1} ; \ldots ; n\left(s_{n}\right) \nu_{n} v_{n}}=\hat{C}_{n\left(r_{1}\right) \mu_{1} ; \ldots n\left(r_{m}\right) \mu_{m}}^{\left(\Sigma u_{i}-\Sigma v_{j}\right)} \quad n\left(s_{1}\right) \nu_{1} ; \ldots ; n\left(s_{n}\right) \nu_{n}
\end{aligned}
$$

and we have checked in detail that the reduced two-component structures are consistent with the formulae of Riemannian geometry. In this demonstration, we found the following formulae to be useful

$$
\begin{aligned}
& \hat{\partial}_{n(r) \mu u} \hat{O}_{n\left(s_{1}\right) \nu_{1} v_{1} ; \ldots ; n\left(s_{m}\right) \nu_{m} v_{m}}^{n\left(t_{1}\right) \delta_{1} w_{1} ; \ldots ;\left(t_{n}\right) \delta_{n} w_{n}}=\hat{C}_{n(r) \mu ; n\left(s_{1}\right) \nu_{1} ; \ldots n\left(s_{m}\right) \nu_{m}}^{\left(u+\Sigma v_{1}-\Sigma w_{j}\right)} \quad{ }^{n\left(t_{1}\right) \delta_{1} ; \ldots ; n\left(t_{n}\right) \delta_{n}} \\
& \hat{\Gamma}_{n(r) \mu ; n(s) \nu}^{(w)}{ }^{n(t) \delta}(\hat{x})=\frac{1}{2} \sum_{u=0}^{1}\left(\hat{\partial}_{n(r) \mu u} \hat{G}_{n(s) \nu ; n(q) \epsilon}^{(w)}(\hat{x})+\hat{\partial}_{n(s) \nu u} \hat{G}_{n(r) \mu ; n(q) \epsilon}^{(w)}(\hat{x})\right. \\
& \left.-\hat{\partial}_{n(q) \epsilon u} \hat{G}_{n(r) \mu ; n(s) \nu}^{(w)}(\hat{x})\right) \hat{G}_{(u)}^{n(q) \epsilon ; n(t) \delta}(\hat{x})
\end{aligned}
$$

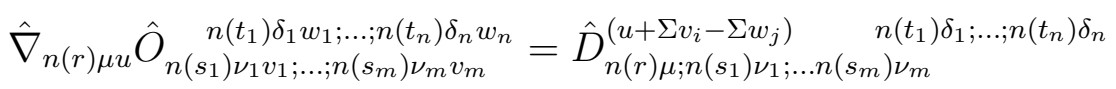

$$
\begin{aligned}
& \hat{\nabla}_{n(r) \mu u} \hat{\phi}_{n(s) \nu v}(\hat{x})=\hat{\partial}_{n(r) \mu 0} \hat{\phi}_{n(s) \nu, u+v}(\hat{x})+\sum_{w=0}^{1} \hat{\Gamma}_{n(r) \mu ; n(s) \nu}^{(u+v-w)} n(t) \delta(\hat{x}) \hat{\phi}_{n(t) \delta w}(\hat{x})
\end{aligned}
$$

where the general relation in (6.25a) follows by local isomorphisms from Eq. (6.19). 
In terms of the reduced quantities, we find the world-sheet parities

$$
\begin{aligned}
& \hat{\nabla}_{n(r) \mu u}(-\xi)=(-1)^{u+1} \hat{\nabla}_{n(r) \mu u}(\xi) \\
& \hat{G}_{(w) \mu ; n(s) \nu}^{n(r)}(\hat{x}(-\xi))=(-1)^{w} \hat{G}_{(w)}^{n(r) \mu ; n(s) \nu}(\hat{x}(\xi)) \\
& \hat{\Gamma}_{n(r) \mu ; n(s) \nu}^{(w)}{ }^{n(t) \delta}(\hat{x}(-\xi))=(-1)^{w+1} \hat{\Gamma}_{n(r) \mu ; n(s) \nu}^{(w)}{ }^{n(t) \delta}(\hat{x}(\xi)) \\
& \hat{R}_{n(r) \mu ; n(s) \nu ; n(t) \delta ; n(q) \epsilon}^{(w)}(\hat{x}(-\xi))=(-1)^{w} \hat{R}_{n(r) \mu ; n(s) \nu ; n(t) \delta ; n(q) \epsilon}^{(w)}(\hat{x}(\xi)) \\
& \hat{R}_{n(r) \mu ; n(s) \nu}^{(w)}(\hat{x}(-\xi))=(-1)^{w} \hat{R}_{n(r) \mu ; n(s) \nu}^{(w)}(\hat{x}(\xi)) \\
& \hat{\phi}_{n(r) \mu u}(\hat{x}(-\xi))=(-1)^{u+1} \phi_{n(r) \mu u}(\hat{x}(\xi)), \quad \bar{u}, \bar{w} \in\{0,1\}
\end{aligned}
$$

which should be considered together with those given earlier in Eq. (5.16).

Following the procedure shown in Fig. 3, we have already given the explicit forms of $\hat{G}, \hat{B}$ and $\hat{H}$ in Eqs. (5.18), (5.19), and we similarly give the explicit forms of the other twisted fields:

$$
\begin{aligned}
& \hat{G}^{n(r) \mu u ; n(s) \nu v}(\hat{x})=G^{i \dot{I} ; j \dot{J}}(\underset{\sigma}{x \rightarrow} \hat{X}(\hat{x})) \chi_{n(r) \mu}^{-1} \chi_{n(s) \nu}^{-1} U_{i}^{\dagger}{ }^{n(r) \mu} U_{j}^{\dagger}{ }_{j}^{n(s) \nu}\left(\frac{1}{\sqrt{2}} U^{\dagger}{ }_{i}^{u}\right)\left(\frac{1}{\sqrt{2}} U^{\dagger}{ }_{j}^{v}\right) \\
& \hat{G}_{(w)}^{n(r) \mu ; n(s) \nu}(\hat{x})=\frac{1}{4}\left(G^{i j}\left(x_{\sigma}^{0} \underset{\sigma}{\rightarrow} \hat{X}^{0}(\hat{x})\right)+(-1)^{w} G^{i j}\left(x_{\sigma}^{1} \underset{\sigma}{\rightarrow} \hat{X}^{1}(\hat{x})\right)\right) \times \\
& \times \chi_{n(r) \mu}^{-1} \chi_{n(s) \nu}^{-1} U_{i}^{\dagger n(r) \mu} U_{j}^{\dagger n(s) \nu} \\
& \hat{\Gamma}_{n(r) \mu u ; n(s) \nu v}{ }^{n(t) \delta w}(\hat{x})=\chi_{n(r) \mu} \chi_{n(s) \nu} \chi_{n(t) \delta}^{-1} U_{n(r) \mu}{ }^{i} U_{n(s) \nu}{ }^{j} U^{\dagger}{ }_{k}{ }^{n(t) \delta} \times \\
& \times\left(\sqrt{2} U_{u}^{\dot{I}}\right)\left(\sqrt{2} U_{v}^{\dot{J}}\right)\left(\frac{1}{\sqrt{2}} U_{\dot{K}}^{\dagger}\right) \Gamma_{i \dot{I} ; j \dot{j}}{ }^{k \dot{K}}(x \underset{\sigma}{\rightarrow} \hat{X}(\hat{x})) \\
& \hat{\Gamma}_{n(r) \mu ; n(s) \nu}^{(w)}{ }^{n(t) \delta}(\hat{x})=\frac{1}{2} \chi_{n(r) \mu} \chi_{n(s) \nu} \chi_{n(t) \delta}^{-1} U_{n(r) \mu}{ }^{i} U_{n(s) \nu}{ }^{j} U^{\dagger}{ }_{k}{ }^{n(t) \delta} \times \\
& \times\left(\Gamma_{i j}^{k}\left(x_{\sigma}^{0} \underset{\sigma}{\rightarrow} \hat{X}^{0}(\hat{x})\right)+(-1)^{w} \Gamma_{i j}^{k}\left(x_{\sigma}^{1} \underset{\sigma}{\rightarrow} \hat{X}^{1}(\hat{x})\right)\right) \\
& \hat{R}_{n(r) \mu u ; n(s) \nu v}(\hat{x})=\chi_{n(r) \mu} \chi_{n(s) \nu} U_{n(r) \mu}{ }^{i} U_{n(s) \nu}{ }^{j}\left(\sqrt{2} U_{u}^{\dot{I}}\right)\left(\sqrt{2} U_{v}^{j}\right) R_{i \dot{I} ; j \dot{J}}(x \rightarrow \hat{\sigma} \hat{X}(\hat{x})) \\
& \hat{R}_{n(r) \mu ; n(s) \nu}^{(w)}(\hat{x})=\chi_{n(r) \mu} \chi_{n(s) \nu} U_{n(r) \mu}^{i} U_{n(s) \nu}^{j}\left(R_{i j}\left(x_{\sigma}^{0} \underset{\sigma}{\rightarrow} \hat{X}^{0}(\hat{x})\right)+(-1)^{w} R_{i j}\left(x_{\sigma}^{1} \underset{\sigma}{\hat{X}^{1}}(\hat{x})\right)\right) \\
& \hat{\phi}_{n(r) \mu u}(\hat{x})=\chi_{n(r) \mu} U_{n(r) \mu}{ }^{i}\left(\sqrt{2} U_{u}^{\dot{I}}\right) \phi_{i \dot{I}}(\underset{\sigma}{x \rightarrow} \hat{x}(\hat{x})) \\
& =\chi_{n(r) \mu} U_{n(r) \mu}^{i}\left(\sqrt{2} U_{u}^{\dot{I}}\right) \phi_{i}\left(x_{\sigma}^{\dot{I}} \rightarrow \hat{X}^{\dot{I}}(\hat{x})\right) \text {. }
\end{aligned}
$$

Here $G^{i j}, \Gamma_{i j}{ }^{k}, R_{i j}$ and $\phi_{i}$ are the original untwisted Einstein fields and $\hat{x}$ is the Einstein coordinate with twisted boundary conditions (see Fig. 3 and Eq. (5.17)). Including the 
results in Eq. (5.18), the general rule for the unreduced explicit forms (with the full set of $n(r) \mu u$ indices) is $x \underset{\sigma}{\longrightarrow} \hat{x}(\hat{x})$ and a factor $\chi U(\sigma) \sqrt{2} U$ or $\chi^{-1} U^{\dagger}(\sigma) \frac{1}{\sqrt{2}} U^{\dagger}$ respectively for each set of down or up indices.

For the special case of the dilaton solution, we obtain the particular form of the orientation-orbifold dilaton vector

$$
\begin{gathered}
\hat{\phi}_{n(r) \mu u}(\hat{x})=\chi_{n(r) \mu} U_{n(r) \mu}{ }^{i}\left(\sqrt{2} U_{u}^{\dot{I}}\right) \frac{\partial}{\partial \hat{X}^{i \dot{I}}} \phi\left(\hat{X}^{\dot{I}}(\hat{x})\right) \\
=\chi_{n(r) \mu} U_{n(r) \mu}{ }^{i}\left(\sqrt{2} U_{u}^{\dot{I}}\right) \frac{\partial}{\partial \hat{X}^{i \dot{I}}}\left(\phi\left(\hat{X}^{0}(\hat{x})\right)+\phi\left(\hat{X}^{1}(\hat{x})\right)\right) \\
=\hat{\partial}_{n(r) \mu u} \hat{\phi}(\hat{x}) \\
\hat{\phi}(\hat{x}) \equiv \phi\left(\hat{x}^{0}(\hat{x})\right)+\phi\left(\hat{X}^{1}(\hat{x})\right)
\end{gathered}
$$

where we used Eqs. (6.7f), 6.27g) to obtain Eq. (6.28a), and Eq. (5.17e to obtain the final form in Eq. (6.28c). Here $\phi(x)$ is the original untwisted dilaton and $\hat{\phi}(\hat{x})$ is the orientationorbifold dilaton. The world-sheet and space-time parities of the orientation-orbifold dilaton

$$
\hat{\phi}(\hat{x}(-\xi))=\hat{\phi}(\hat{x}(\xi)), \quad \hat{\phi}(-\hat{x}(\xi))=\hat{\phi}(\hat{x}(\xi))
$$

follow from the corresponding properties of the orientation-orbifold dilaton vector and the dilaton therefore falls in the class $\hat{O}_{+}$(see Eq. (6.23) ) of orientation-orbifold operators.

\subsection{The Twisted Einstein Equations of Open-String Sector $\hat{h}_{\sigma}$}

The two-component Einstein equations in Eq. (6.7) are easily reexpressed in terms of the corresponding Riemannian eigenfields, and then the principle of local isomorphisms gives the orientation-orbifold Einstein equations

$$
\begin{aligned}
& \hat{R}_{n(r) \mu u ; n(s) \nu v}(\hat{x})+ \\
& +\frac{1}{4} \hat{H}_{n(t) \delta w ; n(r) \mu u}{ }^{n(q) \epsilon x}(\hat{x}) \hat{H}_{n(q) \epsilon x ; n(s) \nu v}^{n(t) \delta w}(\hat{x})-2 \hat{\nabla}_{n(r) \mu u} \hat{\phi}_{n(s) \nu v}(\hat{x})=0 \\
& \left(\hat{\nabla}_{n(t) \delta w}-2 \hat{\phi}_{n(t) \delta w}(\hat{x})\right) \hat{H}_{n(r) \mu u ; n(s) \nu v}^{n(t) \delta w}(\hat{x})=0
\end{aligned}
$$

for each open-string sector $\hat{h}_{\sigma}$ of $A_{M}\left(H_{-}\right) / H_{-}$. When the corresponding untwisted Einstein fields solve the untwisted Einstein equations, the principle of local isomorphisms guarantees that the explicit forms of the twisted fields in Eqs. (5.18) and (6.27) solve the orientationorbifold Einstein equations. 
As always, the orientation-orbifold Einstein equations (6.30) have only two independent components in the two-dimensional space, and the reduced two-component form of this system is

$$
\begin{aligned}
& \hat{R}_{n(r) \mu ; n(s) \nu}^{(w)}(\hat{x})+\frac{1}{2} \sum_{u=0}^{1} \hat{H}_{n(t) \delta ; n(r) \mu}^{(u)}{ }^{n(q) \epsilon}(\hat{x}) \hat{H}_{n(q) \epsilon ; n(s) \nu}^{n(t) \delta}(\hat{x})-2 \hat{\nabla}_{n(r) \mu 0} \hat{\phi}_{n(s) \nu w}(\hat{x})=0 \\
& \hat{\nabla}_{n(t) \delta 0} \hat{H}_{n(r) \mu ; n(s) \nu}^{n(t) \delta}(\hat{x})-\sum_{u=0}^{1} \hat{\phi}_{n(t) \delta u}(\hat{x}) \hat{H}_{n(r) \mu ; n(s) \nu}^{n(t) \delta} \underset{n(w-u)}{(x)}(\hat{x})=0, \quad \bar{w}=0,1
\end{aligned}
$$

where we have used the identity in Eq. (6.25c). The different numerical factors here are the result of performing internal sums on $u, v$ indices.

We know that the orientation-orbifold Einstein equations (6.30), (6.31) hold in each open-string sector $\hat{h}_{\sigma}$ when the original untwisted closed string theory was conformal through one loop. Moreover, Ref. [25] gives a general argument (starting from any conformal closed string theory) that each resulting open-string orientation-orbifold sector $\hat{h}_{\sigma}$ always contains the full orbifold Virasoro algebra

$$
\begin{aligned}
{\left[\hat{L}_{u}\left(m+\frac{u}{2}\right), \hat{L}_{v}\left(n+\frac{v}{2}\right)\right] } & =\left(m-n+\frac{u-v}{2}\right) \hat{L}_{u+v}\left(m+n+\frac{u+v}{2}\right) \\
& +\delta_{m+n+\frac{u+v}{2}, 0} \frac{2 c}{12}\left(m+\frac{u}{2}\right)\left(\left(m+\frac{u}{2}\right)^{2}-1\right)
\end{aligned}
$$

with the characteristically-doubled central charge $\hat{c}=2 c$. Therefore one expects that the orientation-orbifold Einstein system is the necessary and sufficient condition that the openstring orientation-orbifold sector satisfies the orbifold Virasoro algebra through one loop. It would be interesting to check this conclusion explicitly at one loop using the Feynman diagrams associated to the sigma-model orientation-orbifold action (5.20).

\subsection{Monodromies and Boundary Conditions}

When $h_{\sigma}^{2}=1$, the principle of local isomorphisms also gives us the monodromies of all the twisted fields in the orientation-orbifold Einstein equations:

$$
\begin{gathered}
h_{\sigma}^{2}=1: \quad \text { automorphic responses } \underset{\sigma}{\longrightarrow} \text { monodromies } \\
\hat{x}_{\sigma}^{n(r) \mu u}(\xi+2 \pi)=\hat{x}_{\sigma}^{n(r) \mu u}(\xi) e^{2 \pi i\left(\frac{n(r)}{\rho(\sigma)}+\frac{u}{2}\right)}, \quad \hat{\partial}_{n(r) \mu u}(\xi+2 \pi)=e^{-2 \pi i\left(\frac{n(r)}{\rho(\sigma)}+\frac{u}{2}\right)} \hat{\partial}_{n(r) \mu u}(\xi) \\
\hat{G}_{n(r) \mu ; n(s) \nu}^{(w)}(\hat{x}(\xi+2 \pi))=e^{-2 \pi i\left(\frac{n(r)+n(s)}{\rho(\sigma)}+\frac{w}{2}\right)} \hat{G}_{n(r) \mu ; n(s) \nu}^{(w)}(\hat{x}(\xi)) \\
\hat{B}_{n(r) \mu ; n(s) \nu}^{(w)}(\hat{x}(\xi+2 \pi))=e^{-2 \pi i\left(\frac{n(r)+n(s)}{\rho(\sigma)}+\frac{w}{2}\right)} \hat{B}_{n(r) \mu ; n(s) \nu}^{(w)}(\hat{x}(\xi)) \\
\hat{H}_{n(r) \mu ; n(s) \nu ; n(t) \delta}^{(w)}(\hat{x}(\xi+2 \pi))=e^{-2 \pi i\left(\frac{n(r)+n(s)+n(t)}{\rho(\sigma)}+\frac{w}{2}\right)} \hat{H}_{n(r) \mu ; n(s) \nu ; n(t) \delta}^{(w)}(\hat{x}(\xi))
\end{gathered}
$$




$$
\begin{gathered}
\hat{\Gamma}_{n(r) \mu ; n(s) \nu}^{(w)}{ }^{n(t) \delta}(\hat{x}(\xi+2 \pi))=e^{-2 \pi i\left(\frac{n(r)+n(s)-n(t)}{\rho(\sigma)}+\frac{w}{2}\right)} \hat{\Gamma}_{n(r) \mu ; n(s) \nu}^{(w)}{ }^{n(t) \delta}(\hat{x}(\xi)) \\
\hat{R}_{n(r) \mu ; n(s) \nu ; n(t) \delta ; n(q) \epsilon}^{(w)}(\hat{x}(\xi+2 \pi))=e^{-2 \pi i\left(\frac{n(r)+n(s)+n(t)+n(q)}{\rho(\sigma)}+\frac{w}{2}\right)} \hat{R}_{n(r) \mu ; n(s) \nu}^{(w)}(\hat{x}(\xi)) \\
\hat{R}_{n(r) \mu ; n(s) \nu}^{(w)}(\hat{x}(\xi+2 \pi))=e^{-2 \pi i\left(\frac{n(r)+n(s)}{\rho(\sigma)}+\frac{w}{2}\right)} \hat{R}_{n(r) \mu ; n(s) \nu}^{(w)}(\hat{x}(\xi)) \\
\hat{\phi}_{n(r) \mu u}(\hat{x}(\xi+2 \pi))=e^{-2 \pi i\left(\frac{n(r)}{\rho(\sigma)}+\frac{u}{2}\right)} \hat{\phi}_{n(r) \mu u}(\hat{x}(\xi)) \rightarrow \hat{\phi}(\hat{x}(\xi+2 \pi))=\hat{\phi}(\hat{x}(\xi)) .
\end{gathered}
$$

The general rule here, as in space-time orbifold theory, is a negative phase for each down index and a positive phase for each up index. We note in particular that the orientationorbifold dilaton, like the space-time orbifold dilaton [24], has trivial monodromy.

Using the monodromies (6.33) along with the world-sheet parities (5.16), (16.26) in the form

$$
\begin{gathered}
\hat{O}_{ \pm}^{(w)}(\hat{x}(-\xi))= \pm(-1)^{w} \hat{O}_{ \pm}^{(w)}(\hat{x}(\xi)) \\
\hat{O}_{+}=\hat{G}, \hat{H}, \text { the } \hat{R}^{\prime} s, \hat{\phi}, \partial_{t} \hat{O}_{+} \text {and } \partial_{\xi} \hat{O}_{-} \\
\hat{O}_{-}=\hat{x}, \hat{B}, \hat{\Gamma}, \hat{\phi}_{n(r) \mu u}, \partial_{t} \hat{O}_{-} \text {and } \partial_{\xi} \hat{O}_{+}
\end{gathered}
$$

we may derive, as above, the following boundary conditions on the twisted fields:

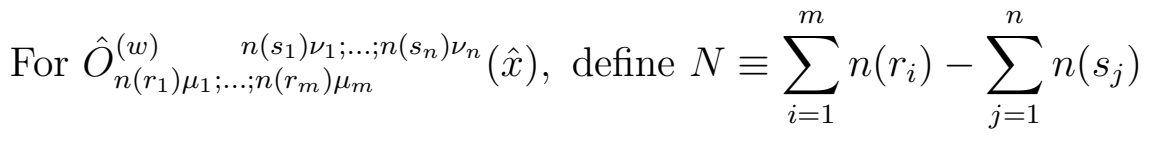

$$
\begin{aligned}
& \text { then } \\
& \hat{O}_{+}^{(1)}(\hat{x}(0)), \hat{O}_{-}^{(0)}(\hat{x}(0))=0 \\
& \hat{O}_{+}^{(w)}(\hat{x}(\pi))=0 \text { unless } \frac{N}{\rho(\sigma)} \in \mathbb{Z} \\
& \hat{O}_{-}^{(w)}(\hat{x}(\pi))=0 \text { unless } \frac{N}{\rho(\sigma)} \in \mathbb{Z}+\frac{1}{2} \text {. }
\end{aligned}
$$

We mention in particular that the orientation-orbifold dilaton $\hat{\phi}$ falls in the class $\hat{O}_{+}$with the specific values $N=w=0$. We finally note that as usual, the boundary conditions (6.35a) at $\xi=0$ follow directly from world-sheet parity alone, and hence hold for all $\hat{h}_{\sigma}$.

\section{Discussion}

In this paper we have provided a description at the action level of the WZW orientation orbifolds, which were constructed at the operator level in Ref. 25. We have moreover extended this classical description to include the action formulation of a large class of sigmamodel orientation orbifolds, and their associated orientation-orbifold Einstein equations.

Directions for future work include the following: 
1) For the WZW orientation orbifolds it may be possible to find more restrictive boundary conditions for the basic fields $\hat{g}$ at $\xi=\pi$ when $h_{\sigma}^{2} \neq 1$, consistent with the variational and twisted current boundary conditions discussed in the text. Such boundary conditions on $\hat{g}$ may follow by a detailed analysis of the classical equations of motion (3.20), using the known monodromies of the currents, as seen for the free bosonic strings in Ref. [25] and Subsec. 5.6.

2) It may similarly be possible to find more restrictive boundary conditions for the basic fields of the coset orientation orbifolds. Additionally, by relaxing our provisional assumption that the WZW orientation-orbifold boundary conditions survive the gauging, more general variational boundary conditions can also be studied.

3) More restrictive boundary conditions on the basic fields of the general sigma-model orientation orbifolds are particularly desirable - because for $h_{\sigma}^{2} \neq 1$ at $\xi=\pi$ we have so far only found the variational boundary conditions. As seen for WZW and the free boson models, possibilities here include study of various sigma-model analogues of the currents.

4) As seen for ordinary orbifolds in Ref. [24], the linear symmetry conditions (5.10) imply selection rules for the coefficients in the moment expansions of the twisted Einstein tensors. In ordinary orbifolds such selection rules are known to connect the monodromies of the twisted Einstein tensors with the monodromy of the twisted Einstein coordinates $\hat{x}$. Although we will not pursue this topic here, the corresponding selection rules for the orientation orbifolds may similarly connect the boundary conditions of the twisted Einstein tensors with those of $\hat{x}$.

\section{Acknowledgements}

For helpful discussions, we thank J. de Boer, O. Ganor, P. Hořava and E. Rabinovici.

This work was supported in part by the Director, Office of Energy Research, Office of High Energy and Nuclear Physics, Division of High Energy Physics of the U.S. Department of Energy under Contract DE-AC03-76SF00098 and in part by the National Science Foundation under grant PHY00-98840.

\section{A Useful Identities}

In the computations of the text, we found the following identities useful

$$
\rho_{0}=\left(\begin{array}{ll}
1 & 0 \\
0 & 0
\end{array}\right), \quad \rho_{1}=\left(\begin{array}{ll}
0 & 0 \\
0 & 1
\end{array}\right), \quad \tau_{1} \rho_{\dot{I}} \tau_{1}=\mathbb{1}-\rho_{\dot{I}}=\left(\tau_{1}\right)_{\dot{I} j} \rho_{j}
$$




$$
\begin{gathered}
\left(\tau_{1}\right)_{\dot{I}}^{\dot{K}}\left(\tau_{1}\right)_{\dot{j}}^{\dot{K}}=\delta_{\dot{I} \dot{J}}\left(\tau_{1}\right)_{\dot{I}}^{\dot{K}}, \quad \dot{I}, \dot{J}, \dot{K} \in\{0,1\} \\
\sqrt{2} \sum_{\dot{I}=0}^{1} U_{u}{ }^{\dot{I}} U \rho_{\dot{I}} U^{\dagger}=\tau_{u}, \quad \sqrt{2} \sum_{\dot{I}=0}^{1} U_{u}{ }^{\dot{I}} U\left(1-\rho_{\dot{I}}\right) U^{\dagger}=(-1)^{u} \tau_{u}, \quad u=0,1 \\
U \tau_{1}=\tau_{3} U, \quad \sqrt{2} \sum_{\dot{I}=0}^{1} U_{u}^{\dot{I}} U_{v}{ }^{\dot{I}}\left(U^{\dagger}\right)_{\dot{I}}{ }^{w}=\delta_{u+v+w, 0 \bmod 2} \\
2^{n / 2} \sum_{\dot{I}=0}^{1} U_{u_{1}}{ }^{\dot{I}} \cdots U_{u_{n}}{ }^{\dot{I}} f\left(x^{\dot{I}}\right)=f\left(x^{0}\right)+(-1)^{\sum_{i=1}^{n} u_{i}} f\left(x^{1}\right)
\end{gathered}
$$

where $\vec{\tau}$ are the Pauli matrices and $\tau_{0}=\mathbb{1}$ is the $2 \mathrm{x} 2$ unit matrix.

\section{B Space-Time Parity in Coset CFTs}

In this appendix, we will study the space-time parities of the Einstein fields $G, B$ and the dilaton $\phi$ for any $g / h$ coset CFT [6, 7, 33, 12] with $G / H$ a reductive coset space.

We begin with the well-known form [39-43] of the total $G$ and $B$ fields in the coset CFT:

$$
\begin{gathered}
(G(x)+B(x))_{i j}^{t o t}=(G(x)+B(x))_{i j}+\Delta(G(x)+B(x))_{i j} \\
\Delta(G(x)+B(x))_{i j} \equiv-2\left(e(x)_{i}{ }^{\hat{a}} G_{\hat{a} \hat{c}}\right)\left(\bar{e}(x)_{j}{ }^{\hat{b}} G_{\hat{b} \hat{d}}\right) X^{-1}(x)^{\hat{c} \hat{d}} \\
X(x)_{\hat{a} \hat{b}} \equiv G_{\hat{a} \hat{b}}-\Omega(x)_{\hat{a} \hat{b}}, \quad \Omega(x)_{a b}=\Omega(x)_{a}{ }^{c} G_{c b} \\
i, j=1 \ldots \operatorname{dim} g, \quad a, b=1 \ldots \operatorname{dim} g, \quad \hat{a}, \hat{b}=1 \ldots \operatorname{dim} h .
\end{gathered}
$$

Here the quantities $G(x), B(x), e(x), \bar{e}(x)$ and $\Omega(x)$ are the standard WZW quantities in Eq. (4.1). The tangent-space metric $G_{a b}$ of $g$ is block diagonal in $h$ and $g / h$ and $G_{\hat{a} \hat{b}}$ is the invertible induced metric on $h \subset g$. We already know (see Eq. (4.6b) ) that the WZW metric and $B$ field exhibit the space-time parities

$$
G_{i j}(-x)=G_{i j}(x), \quad B_{i j}(-x)=-B_{i j}(x)
$$

so it is only necessary to study the extra contributions $\Delta(G+B)$ in (B.1b) .

In this discussion the following identities are useful

$$
\begin{gathered}
\Omega^{T}(x)_{a c}=\Omega(x)_{c a}=\Omega(x)_{c}{ }^{d} G_{d a}=\Omega^{-1}(x)_{a}{ }^{d} G_{d c}=\Omega^{-1}(x)_{a c} \\
\Omega(-x)_{a b}=\Omega^{-1}(x)_{a b}=\Omega^{T}(x)_{a b} \\
e(-x)_{i}{ }^{a}=-\bar{e}(x)_{i}{ }^{a}, \quad \bar{e}(-x)_{i}{ }^{a}=-e(x)_{i}{ }^{a}
\end{gathered}
$$


where superscript $T$ is transpose. Because $G / H$ is reductive, all these relations can be restricted to $h$, e. g.

$$
\Omega(x)_{\hat{c}}^{\hat{d}} G_{\hat{d} \hat{a}}=\Omega^{-1}(x)_{\hat{a}}^{\hat{d}} G_{\hat{d} \hat{c}}
$$

and it follows that:

$$
X(-x)_{\hat{a} \hat{b}}=X^{T}(x)_{\hat{a} \hat{b}}, \quad X^{-1}(-x)^{\hat{a} \hat{b}}=X^{-1 T}(x)^{\hat{a} \hat{b}} .
$$

Then we may compute

$$
\begin{aligned}
\Delta(G(-x)+B(-x))_{i j} & =-2\left(e(-x)_{i}{ }^{\hat{a}} G_{\hat{a} \hat{c}}\right)\left(\bar{e}(-x)_{j}{ }^{\hat{b}} G_{\hat{b} \hat{d}}\right) X^{-1}(-x)^{\hat{c} \hat{d}} \\
& =-2\left(\bar{e}(x)_{i}{ }^{\hat{a}} G_{\hat{a} \hat{c}}\right)\left(e(x)_{j}{ }^{\hat{b}} G_{\hat{b} \hat{d}}\right) X^{-1 T}(x)^{\hat{c} \hat{d}} \\
& =-2\left(e(x)_{j}{ }^{\hat{a}} G_{\hat{a} \hat{c}}\right)\left(\bar{e}(x)_{i}{ }^{\hat{b}} G_{\hat{b} \hat{d}}\right) X^{-1}(x)^{\hat{c} \hat{d}} \\
& =\Delta(G(x)+B(x))_{j i}
\end{aligned}
$$

and this result implies the space-time parities of $\Delta G$ and $\Delta B$ separately

$$
\Delta G_{i j}(-x)=\Delta G_{i j}(x), \quad \Delta B_{i j}(-x)=-\Delta B_{i j}(x)
$$

because they are respectively the symmetric and antisymmetric parts of $\Delta(G+B)$.

The final result for the space-time parities of the total coset $G$ and $B$

$$
G_{i j}^{t o t}(-x)=G_{i j}^{t o t}(x), \quad B_{i j}^{t o t}(-x)=-B_{i j}^{t o t}(x)
$$

then follows immediately from Eqs. (B.2) and (B.7).

The formula for the coset dilaton is also well-known [39-43,50]

$$
\phi(x)=-\frac{1}{2} \ln \operatorname{det}(X(x)), \quad \phi_{i}(x) \equiv \nabla_{i} \phi(x)=\partial_{i} \phi(x)
$$

where this $\phi_{i}$ is the special dilaton vector associated to the dilaton. The space-time parities of the dilaton and dilaton vector

$$
\phi(-x)=-\frac{1}{2} \ln \operatorname{det}\left(X^{T}(x)\right)=\phi(x), \quad \phi_{i}(-x)=-\phi_{i}(x)
$$

follow immediately from Eq. (B.5).

These space-time parities and the discussion of the Subsecs. 5.1 and 6.1 show explicitly that all (reductive) $g / h$ coset CFTs admit the basic orientation-reversing automorphism, at least through one loop. We know of course from the operator form of the coset orientationorbifold stress tensors in Eq. (5.34) that the basic orientation-reversal is an automorphism of the coset CFT to all orders. 


\section{References}

[1] L. Borisov, M. B. Halpern, and C. Schweigert, "Systematic approach to cyclic orbifolds," Int. J. Mod. Phys. A13 (1998) 125, hep-th/9701061.

[2] J. Evslin, M. B. Halpern, and J. E. Wang, "General Virasoro construction on orbifold affine algebra," Int. J. Mod. Phys. A14 (1999) 4985, hep-th/9904105

[3] J. de Boer, J. Evslin, M. B. Halpern, and J. E. Wang, "New duality transformations in orbifold theory," Int. J. Mod. Phys. A15 (2000) 1297, hep-th/9908187.

[4] J. Evslin, M. B. Halpern, and J. E. Wang, "Cyclic coset orbifolds," Int. J. Mod. Phys. A15 (2000) $3829-3860$, hep-th/9912084

[5] M. B. Halpern and J. E. Wang, "More about all current-algebraic orbifolds," Int. J. Mod. Phys. A16 (2001) 97, hep-th/0005187.

[6] K. Bardakci and M. B. Halpern, "New dual quark models," Phys. Rev. D3 (1971) 2493.

[7] M. B. Halpern, "The two faces of a dual pion-quark model," Phys. Rev. D4 (1971) 2398.

[8] M. B. Halpern and C. B. Thorn, "Two faces of a dual pion-quark model. II. Fermions and other things," Phys. Rev. D4 (1971) 3084.

[9] M. B. Halpern, "Quantum 'Solitons' which are su(N) Fermions," Phys. Rev D12 (1975) 1684; "Equivalent-Boson Method and Free Currents in Two-Dimensional Gauge Theories," Phys. Rev. D13 (1976) 337.

[10] M. B. Halpern and E. Kiritsis, "General Virasoro construction on affine g," Mod. Phys. Lett. A4 (1989) 1373.

[11] M. B. Halpern, E. B. Kiritsis, and N. A. Obers, "The Lie $h$ invariant conformal field theories and the Lie $h$ invariant graphs," Int. J. Mod. Phys. A7 (Suppl. 1A) (1992) 339, hep-th/9110001

[12] M. B. Halpern, E. Kiritsis, N. A. Obers, and K. Clubok, "Irrational conformal field theory," Physics Reports 265, Nos. 1 \& 2, (1996) 1-138, hep-th/9501144

[13] J. de Boer and M. B. Halpern, "Unified Einstein-Virasoro Master Equation in the Nonlinear Sigma Model," Int. J. Mod. Phys. A12 (1997) 1551, hep-th/9606025

[14] J. de Boer, M. B. Halpern, and N. A. Obers, "The operator algebra and twisted KZ equations of WZW orbifolds," JHEP 10 (2001) 011, hep-th/0105305

[15] M. B. Halpern and N. A. Obers, "Two Large Examples in Orbifold Theory: Abelian Orbifolds and the Charge Conjugation Orbifold on su(n)," Int. J. Mod. Phys. A17 (2002) 3897, hep-th/0203056

[16] M. B. Halpern and C. Helfgott, "Extended Operator Algebra and Reducibility in the WZW Permutation Orbifolds," Int. J. Mod Phys. A18 (2003) 1774, hep-th/0208087

[17] O. Ganor, M. B. Halpern, C. Helfgott and N. A. Obers, "The Outer-Automorphic WZW Orbifolds on $\mathfrak{s o}(2 n)$, including Five Triality Orbifolds on $\mathfrak{s o}(8), "$ J. High Energy Phys. 0212 (2002) 019, hep-th/0211003.

[18] J. Fröhlich, O. Grandjean, A. Recknagel and V. Schomerus, "Fundamental strings in Dp-Dq brane systems," Nucl. Phys. B583 (2000) 381, hep-th/9912079.

[19] R. Dijkgraaf, E. Verlinde and H. Verlinde, "Matrix String Theory," Nucl. Phys. B 500 (1997) 43, hep-th/9703030

[20] V. G. Kac and I. T. Todorov, "Affine orbifolds and rational conformal field theory extensions of $W_{1+\infty}, "$ Comm. Math. Phys. 190 (1997) 57, hep-th/9612078.

[21] P. Bantay, "Characters and modular properties of permutation orbifolds," Phys. Lett. B419 (1998) 175-178, hep-th/9708120.

[22] L. Birke, J. Fuchs, and C. Schweigert, "Symmetry breaking boundary conditions and WZW orbifolds," Adv. Theor. Math. Phys. 3 (1999) 671-726, hep-th/9905038.

[23] M. B. Halpern and F. Wagner, "The General Coset Orbifold Action," Int. J. Mod. Phys. A18 (2003) 19, hep-th/0205143 
[24] J. deBoer, M. B. Halpern and C. Helfgott, "Twisted Einstein Tensors and Orbifold Geometry," Int. J. Mod. Phys. A18 (2003) 3489, hep-th/0212275

[25] M. B. Halpern and C. Helfgott, "Twisted Open Strings from Closed Strings: The WZW Orientation Orbifolds," hep-th/0306014. To appear in IJMPA.

[26] S. Giusto and M. B. Halpern, "Hamiltonian Formulation of Open WZW Strings," Int. J. Mod. Phys. A16 (2001) 3237, hep-th/0101220

[27] V. Kac, "Simple graded Lie algebras of finite growth," Funct. Anal. Appl. 1 (1967) 328.

[28] R. V. Moody, "Lie algebras associated with generalized Cartan matrices," Bull. Am. Math. Soc. 73 (1967) 217-221.

[29] S. P. Novikov, Usp. Math. Nauk. 37 (1982) 3.

[30] E. Witten, "Non-Abelian Bosonization in Two Dimensions," Comm. Math. Phys. 92 (1984) 455.

[31] M. B. Halpern and J. P. Yamron, Nucl. Phys. B351 (1991) 333.

[32] K. Clubok and M. B. Halpern, Int. J. Mod. Phys. A11 (1996) 2167.

[33] P. Goddard, A. Kent, and D. I. Olive, "Virasoro Algebras and Coset Space Models," Phys. Lett. B152, (1985) 88.

[34] E. Corrigan and D. B. Fairlie, "Off-Shell States in Dual Resonance Theory," Nucl. Phys B91 (1975) 527.

[35] W. Siegel, "Strings with Dimension-Dependent Intercept," Nucl. Phys B109 (1976) 244.

[36] R. Dashen and Y. Frishman, "Four fermion interactions and scale invariance," Phys. Rev. D11 (1975) 2781.

[37] V. G. Knizhnik and A. B. Zamolodchikov, "Current Algebra and Wess-Zumino Model in Two Dimensions," Nucl. Phys. B247 (1984) 83.

[38] G. Segal, unpublished.

[39] K. Bardakci, E. Rabinovici, and B. Säring, "String models with $c<1$ components," Nucl. Phys. B299, (1988) 151.

[40] K. Gawedzki and A. Kupiainen, "G/H conformal field theory from gauged WZW models" Phys. Lett. B215, (1988) 119.

[41] K. Gawedzki and A. Kupiainen, "Coset construction from functional integrals," Nucl. Phys. B320, (1989) 625.

[42] D. Karabali, Q.-H. Park, H. J. Schnitzer, and Z. Yang, "A GKO construction based on a path integral formulation of gauged Wess-Zumino-Witten actions," Phys. Lett. B216, (1989) 307.

[43] D. Karabali and H. J. Schnitzer, "BRST quantization of the gauged WZW action and coset conformal field theories," Nucl. Phys. B329, (1990) 649.

[44] D. Friedan, "Nonlinear Models in $2+\epsilon$ dimensions," Ph.D. thesis, LBL-11517 (1980); Phys. Rev. Lett. 45, (1980) 1057.

[45] L. Alvarez-Gaumé, D. Z. Freedman and S. Mukhi, Ann. Phys. 134 (1981) 85.

[46] C. Lovelace, Phys. Lett. B135 (1984) 75.

[47] E. S. Fradkin and A. A. Tseytlin, Nucl. Phys. B261 (1985) 1.

[48] C. G. Callan, D. Friedan, E. J. Martinec and M. J. Perry, Nucl. Phys. B262 (1985) 593.

[49] A. A. Tseytlin, Int. J. Mod. Phys. A4 (1989) 1257.

[50] I. Bars and K. Sfetsos, "Exact Effective Action and Spacetime Geometry in Gauged WZW Models", Phys. Rev. D48 (1993) 844, hep-th/9301047 